\title{
Synthesis of the Prototypical Cyclopropyl Dipeptide Mimic and Evaluation of Its Turn-Inducing Capability
}

Albane Neckebroeck, Sharon Kelly, Brian Smith and J. Stephen Clark*

\section{Supporting Information}

\section{Table of Contents}

General Information

Experimental Procedures for the Preparation of $\mathbf{1 3}$

${ }^{1} \mathrm{H}$ and ${ }^{13} \mathrm{C}$ NMR Spectra for New Compounds

Concentration-Dependent NMR Data (Figures 3, 5 and 6)

Amide N-H IR Data (Figures 3, 5 and 6)

SI-76

Circular Dichroism Data (Figure 8) 


\section{General Information}

Peptides were purified using a Gilson semi-preparative HPLC system equipped with a Phenomenex Synergi $10 \mu \mathrm{C} 1880 \AA$, $(250 \times 21.2 \mathrm{~mm})$ column. Peptides were lyophilized using a Christ Alpha 24 LDplus freeze dryer.

IR spectroscopy was performed on a solution ( $10 \mathrm{mM}$ in $\mathrm{CH}_{2} \mathrm{Cl}_{2}$ ) contained in a liquid cell and spectra were recorded using a Shimadzu FTIR-8400S spectrometer.

NMR spectra were recorded using a Bruker Avancelll $400 \mathrm{MHz}$, Bruker Avancelll UltraShield 500 $\mathrm{MHz}$ or a Bruker UltraShield $600 \mathrm{MHz}$ spectrometer.

Far-UV circular dichroism (CD) spectra were acquired using a Jasco J-810 spectropolarimeter with a cell path length of $0.1 \mathrm{~cm}$ and a sample concentration of $1 \mathrm{mg} / \mathrm{mL}$.

High resolution mass spectra (HRMS) were recorded using chemical ionization ( $\mathrm{Cl}$ ) and electron impact ionization (EI) on a Joel MStation JMS-700 instrument or using electrospray ionization (ESI; positive or negative mode) on a Bruker micrOTOF-Q instrument.

Melting points were obtained using a Barnstead Electrothermal 9100 melting point apparatus. In cases where no solvent is indicated, the solid provided by the described procedure was melted directly without recrystallisation.

Optical rotations $\left([\alpha]_{\mathrm{D}}\right)$ were determined using a Rudolph Research Analytical Autopol IV or V digital polarimeter. 


\section{Experimental Procedures for the Preparation of 13}

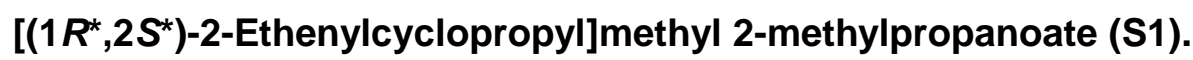

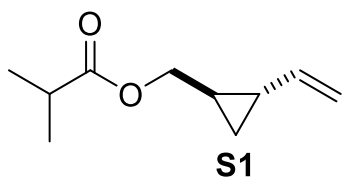

To a cooled solution of dimethylaminopyridine $(24.0 \mathrm{mg}, 0.196 \mathrm{mmol})$ in pyridine $(5.00 \mathrm{~mL})$ a $0{ }^{\circ} \mathrm{C}$, was added the alcohol ( \pm )-5 (1.00 g, $10.2 \mathrm{mmol})$. Isobutyric anhydride $(3.40 \mathrm{~mL}, 20.4 \mathrm{mmol})$ was added dropwise to the solution and the reaction mixture was stirred at rt for $48 \mathrm{~h}$. Water $(15 \mathrm{~mL})$ was added and the resulting aqueous solution was extracted with ether $(3 \times 15 \mathrm{~mL})$. The combined organic extracts were washed with sat. aq. ammonium chloride $(10 \mathrm{~mL})$, water $(10 \mathrm{~mL})$ and brine $(10 \mathrm{~mL}$ ), then dried (magnesium sulfate) and concentrated under vacuum. The residue was purified by column chromatography on silica gel (pet. ether-ethyl acetate, 20:1) to afford the ester $\mathbf{S 1}$ (819 $\mathrm{mg}, 48 \%)$ as an oil. $\mathrm{V}_{\max }\left(\mathrm{CHCl}_{3}\right) 2972,2362,1732,1190,1152,897 \mathrm{~cm}^{-1} ;{ }^{1} \mathrm{H} \mathrm{NMR}\left(500 \mathrm{MHz}, \mathrm{CDCl}_{3}\right)$ $\delta 5.39(1 \mathrm{H}$, ddd, $J=17.0,10.3,8.5 \mathrm{~Hz}), 5.05(1 \mathrm{H}, \mathrm{dd}, J=17.0,1.5 \mathrm{~Hz}), 4.87(1 \mathrm{H}, \mathrm{dd}, J=10.3,1.5$ Hz), $3.97(1 \mathrm{H}, \mathrm{dd}, J=11.3,6.9 \mathrm{~Hz}), 3.94(1 \mathrm{H}, \mathrm{dd}, J=11.3,6.9 \mathrm{~Hz}), 2.54(1 \mathrm{H}, \mathrm{p}, J=7.0 \mathrm{~Hz}), 1.45-$ $1.34(1 \mathrm{H}, \mathrm{m}), 1.22-1.17(1 \mathrm{H}, \mathrm{m}), 1.16(6 \mathrm{H}, \mathrm{d}, J=7.0 \mathrm{~Hz}), 0.72(1 \mathrm{H}, \mathrm{ddd}, J=8.5,5.3,5.3 \mathrm{~Hz}), 0.67$ $(1 \mathrm{H}$, ddd, $J=8.5,5.1,5.1 \mathrm{~Hz}) ;{ }^{13} \mathrm{C}\{1 \mathrm{H}\} \mathrm{NMR}\left(126 \mathrm{MHz}, \mathrm{CDCl}_{3}\right) \delta 177.4,140.3,112.8,67.5,34.2$, 20.9, 19.4, 19.1, 12.0; HRMS (ESI) for $\mathrm{C}_{10} \mathrm{H}_{16} \mathrm{O}_{2} \mathrm{Na}[\mathrm{M}+\mathrm{Na}]^{+}$calcd. 191.1043, found 191.1043.

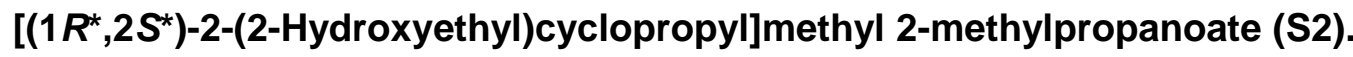

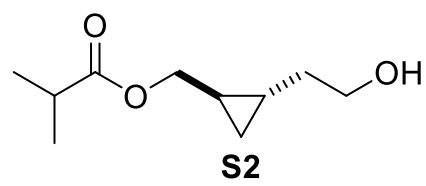

A flask equipped with an argon inlet was charged with borane-THF complex $(10.0 \mathrm{~mL}$ of a $1 \mathrm{~m}$ solution in THF, $9.74 \mathrm{mmol})$. A solution of the alkene $\mathbf{S 1}(819 \mathrm{mg}, 4.87 \mathrm{mmol})$ in THF (18 mL) was added dropwise at $0{ }^{\circ} \mathrm{C}$. The mixture was stirred at this temperature for $4 \mathrm{~h}$. Water $(9 \mathrm{~mL})$ was then added carefully followed by $\mathrm{pH} 7$ phosphate buffer solution $(9 \mathrm{~mL})$. Sodium perborate $(2.20 \mathrm{~g}, 14.6$ $\mathrm{mmol}$ ) was added and the reaction mixture was stirred for a further $2 \mathrm{~h}$. The excess of sodium perborate was removed by filtration and the solvent was removed under vacuum. The aqueous phase was extracted with ether $(3 \times 20 \mathrm{~mL})$. The combined organic extracts were washed with brine $(20 \mathrm{~mL})$, dried (magnesium sulfate) and concentrated under vacuum. The residue was purified by column chromatography on silica gel (pet. ether-ethyl acetate, 1:1) to give the alcohol S2 (83 mg, 9\%) as an oil. $v_{\max }\left(\mathrm{CHCl}_{3}\right) 3422,2931,1732,1471,1192,1155,1072,1044 \mathrm{~cm}^{-1} ;{ }^{1} \mathrm{H}$ NMR $(500$ $\left.\mathrm{MHz}, \mathrm{CDCl}_{3}\right) \delta 4.09(1 \mathrm{H}, \mathrm{dd}, J=11.4,6.2 \mathrm{~Hz}), 3.75(1 \mathrm{H}, \mathrm{dd}, J=11.4,8.3 \mathrm{~Hz}), 3.69(2 \mathrm{H}, \mathrm{ddd}, J=$ 6.8, 6.1, 2.4 Hz), $2.55(1 \mathrm{H}$, hept, $J=7.0 \mathrm{~Hz}), 1.65(1 \mathrm{H}$, dddd, $J=13.5,12.8,6.1,6.1 \mathrm{~Hz}), 1.36(1 \mathrm{H}$, dddd, $J=14.3,12.8,6.8,6.8 \mathrm{~Hz}), 1.17(3 \mathrm{H}, \mathrm{d}, J=7.0 \mathrm{~Hz}), 1.17(3 \mathrm{H}, \mathrm{d}, J=7.0 \mathrm{~Hz}), 1.00-0.87(1 \mathrm{H}$, m), 0.80-0.69 (1H, m), $0.49(1 \mathrm{H}$, ddd, $J=8.5,4.9,4.9 \mathrm{~Hz}), 0.42(1 \mathrm{H}$, ddd, $J=8.5,5.1,5.1 \mathrm{~Hz})$; 
${ }^{13} \mathrm{C}\{1 \mathrm{H}\} \operatorname{NMR}\left(126 \mathrm{MHz}, \mathrm{CDCl}_{3}\right) \delta 177.5,68.6,63.0,36.4,34.2,19.2,17.1,14.7,9.7 ; \mathrm{HRMS}$ (ESI) for $\mathrm{C}_{10} \mathrm{H}_{18} \mathrm{O}_{3} \mathrm{Na}[\mathrm{M}+\mathrm{Na}]^{+}$calcd. 209.1148, found 209.1150 .

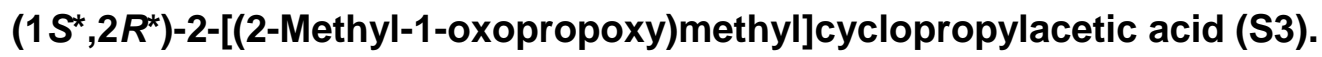<smiles>CC(C)C(=O)OCC1[As]C1CC(=O)O</smiles>

The alcohol S2 (83 mg, $0.45 \mathrm{mmol})$ and $N$-methylmorpholine- $N$-oxide monohydrate $(527 \mathrm{mg}, 4.50$ $3.90 \mathrm{mmol}$ ) were dissolved in acetonitrile $(2 \mathrm{~mL})$. Tetra- $n$-propylammonium perruthenate $(18 \mathrm{mg}$, $0.051 \mathrm{mmol}, 10 \mathrm{~mol} \%)$ was added at $\mathrm{rt}$ and the mixture was stirred at $\mathrm{rt}$ overnight. The reaction was quenched by the addition of excess of isopropanol. Water $(5 \mathrm{~mL})$ was added and the $\mathrm{pH}$ was carefully adjusted to 1 using $2 \mathrm{~m}$ aq. hydrochloric acid. The aqueous phase was extracted with ether $(3 \times 10$ $\mathrm{mL}$ ) and the combined organic extracts were washed with brine $(10 \mathrm{~mL})$ and dried (magnesium sulfate). Removal of the solvent under vacuum afforded the carboxylic acid S3 (95 $\mathrm{mg}$ ) as a dark oil, which was used in the subsequent reaction without purification. ${ }^{1} \mathrm{H} \mathrm{NMR}\left(500 \mathrm{MHz}, \mathrm{CDCl}_{3}\right) \delta 3.97$ $(1 \mathrm{H}, \mathrm{dd}, J=11.5,6.7 \mathrm{~Hz}), 3.92(1 \mathrm{H}, \mathrm{dd}, J=11.6,7.0 \mathrm{~Hz}), 2.54(1 \mathrm{H}$, hept, $J=7.0 \mathrm{~Hz}), 2.30(2 \mathrm{H}$, dd, $J=14.0,6.9 \mathrm{~Hz}), 1.16(3 \mathrm{H}, \mathrm{d}, J=7.1 \mathrm{~Hz}), 1.16(3 \mathrm{H}, \mathrm{d}, J=7.1 \mathrm{~Hz}), 1.09-1.00(2 \mathrm{H}, \mathrm{m}), 0.60(1 \mathrm{H}$, ddd, $J=8.3,5.3,5.3 \mathrm{~Hz}), 0.50(1 \mathrm{H}$, ddd, $J=8.3,5.3,5.3 \mathrm{~Hz}) ;{ }^{13} \mathrm{C}\{1 \mathrm{H}\} \mathrm{NMR}\left(126 \mathrm{MHz}, \mathrm{CDCl}_{3}\right) \delta$ 178.3, 177.4, 67.6, 38.1, 34.2, 19.1, 19.1, 17.4, 12.9, 10.2; HRMS (ESI) for $\mathrm{C}_{10} \mathrm{H}_{16} \mathrm{O}_{4} \mathrm{Na}[\mathrm{M}+\mathrm{Na}]^{+}$ calcd. 223.0941, found 223.0944.

$\left(1 S^{\star}, 2 R^{\star}\right)-2-[(2-M e t h y l-1-0 x o p r o p o x y)$ methyl]cyclopropyl-N-isopropylacetamide (13).<smiles>CC(C)NC(=O)C[C@@H]1[C@@H](COC(=O)C(C)C)[C@@H]1C</smiles>

The carboxylic acid S3 (95 $\mathrm{mg}, 0.47 \mathrm{mmol})$, isopropylamine $(81 \mu \mathrm{L}, 0.99 \mathrm{mmol})$ and triethylamine (230 $\mu \mathrm{L}, 1.65 \mathrm{mmol})$ were dissolved in $\mathrm{CH}_{2} \mathrm{Cl}_{2}(2.5 \mathrm{~mL})$. The solution was stirred at $\mathrm{rt}$ for $15 \mathrm{~min}$ and T3P $(0.42 \mathrm{~mL}$, of a $50 \%$ solution in ethyl acetate, $0.66 \mathrm{mmol})$ was added dropwise. The reaction mixture was stirred at $\mathrm{rt}$ for $18 \mathrm{~h}$. The solution was washed with water $(3 \times 10 \mathrm{~mL})$ and the phases were separated. The organic phase was dried (magnesium sulfate) and concentrated under vacuum to give the amide 13 (55 mg, 48\%) as a colourless solid. m.p. $>400{ }^{\circ} \mathrm{C} ; \mathrm{v}_{\max }\left(10 \mathrm{mM}\right.$ in $\left.\mathrm{CH}_{2} \mathrm{Cl}_{2}\right)$ 3429, 3059, 2970, 2928, 2870, 1724, 1663, 1512, 1466, 1270, 1161, $1157 \mathrm{~cm}^{-1}$; ${ }^{1} \mathrm{H}$ NMR $(500 \mathrm{MHz}$, $\left.\mathrm{CDCl}_{3}\right) \delta 5.71(1 \mathrm{H}, \mathrm{br} \mathrm{s}), 4.14-4.10(1 \mathrm{H}, \mathrm{m}), 4.08(1 \mathrm{H}, \mathrm{dd}, J=11.6,6.4 \mathrm{~Hz}), 3.81(1 \mathrm{H}, \mathrm{dd}, J=11.6$, $7.6 \mathrm{~Hz}), 2.55(1 \mathrm{H}$, hept, $J=7.0 \mathrm{~Hz}), 2.18(1 \mathrm{H}, \mathrm{dd}, J=16.1,6.9 \mathrm{~Hz}), 2.11(1 \mathrm{H}, \mathrm{dd}, J=16.1,7.3 \mathrm{~Hz})$, 1.18-1.13 (12H, m), 1.05-0.90 (2H, m), $0.59(1 \mathrm{H}, \mathrm{ddd}, J=8.4,5.0,5.0 \mathrm{~Hz}), 0.48(1 \mathrm{H}$, ddd, $J=8.4$, $5.1,5.1 \mathrm{~Hz}) ;{ }^{13} \mathrm{C}\{1 \mathrm{H}\} \mathrm{NMR}\left(126 \mathrm{MHz}, \mathrm{CDCl}_{3}\right) \delta 177.4,170.9,67.8,41.4,40.7,34.1,22.9,19.2,17.5$, 13.6, 10.2; HRMS (ESI) for $\mathrm{C}_{13} \mathrm{H}_{23} \mathrm{NO}_{3} \mathrm{Na}[\mathrm{M}+\mathrm{Na}]^{+}$calcd. 264.1570, found 264.1572. 


\section{${ }^{1} \mathrm{H}$ and ${ }^{13} \mathrm{C}$ NMR Spectra for New Compounds}

${ }^{1} \mathrm{H}$ NMR Spectrum of $( \pm)-5 \quad\left(400 \mathrm{MHz}, \mathrm{CDCl}_{3}\right)$

Page

${ }^{13} \mathrm{C}\left\{{ }^{1} \mathrm{H}\right\}$ NMR Spectrum of $( \pm)-5 \quad\left(101 \mathrm{MHz}, \mathrm{CDCl}_{3}\right)$

SI-7

${ }^{1} \mathrm{H}$ NMR Spectrum of $( \pm)-6$

$\left(400 \mathrm{MHz}, \mathrm{CDCl}_{3}\right)$

SI-8

${ }^{13} \mathrm{C}\left\{{ }^{1} \mathrm{H}\right\}$ NMR Spectrum of $( \pm)-6 \quad\left(126 \mathrm{MHz}, \mathrm{CDCl}_{3}\right)$

SI-9

${ }^{1} \mathrm{H}$ NMR Spectrum of (1R,2S)-2-Ethenyl- $N-[(R)$-2-hydroxy-1-phenylethyl]-

cyclopropane-1-carboxamide $\quad\left(500 \mathrm{MHz}, \mathrm{CDCl}_{3}\right)$

SI-11

${ }^{13} \mathrm{C}\left\{{ }^{1} \mathrm{H}\right\}$ NMR Spectrum of $(1 R, 2 S)-2-E$ thenyl- $N$-[(R)-2-hydroxy-1-phenylethyl]-

cyclopropane-1-carboxamide

$\left(126 \mathrm{MHz}, \mathrm{CDCl}_{3}\right.$ )

SI-12

${ }^{1} \mathrm{H}$ NMR Spectrum of $(1 R, 2 S)$-2-Ethenyl- $N-[(R)-2$-hydroxy-1-phenylethyl]-

cyclopropane-1-carboxamide

$\left(500 \mathrm{MHz}, \mathrm{CDCl}_{3}\right)$

$\mathrm{SI}-13$

${ }^{13} \mathrm{C}\left\{{ }^{1} \mathrm{H}\right\}$ NMR Spectrum of $(1 R, 2 S)-2-$ Ethenyl- $N-[(R)-2$-hydroxy-1-phenylethyl]-

cyclopropane-1-carboxamide

${ }^{1} \mathrm{H}$ NMR Spectrum of $(-)-6$

${ }^{13} \mathrm{C}\left\{{ }^{1} \mathrm{H}\right\}$ NMR Spectrum of $(-)-6$

${ }^{1} \mathrm{H}$ NMR Spectrum of $(+)-6$

${ }^{13} \mathrm{C}\left\{{ }^{1} \mathrm{H}\right\}$ NMR Spectrum of $(+)-6$

${ }^{1} \mathrm{H}$ NMR Spectrum of (-)-5

${ }^{13} \mathrm{C}\left\{{ }^{1} \mathrm{H}\right\}$ NMR Spectrum of $(-)-5$

${ }^{1} \mathrm{H}$ NMR Spectrum of $(+)-5$

${ }^{13} \mathrm{C}\left\{{ }^{1} \mathrm{H}\right\}$ NMR Spectrum of $(+)-5$

${ }^{1} \mathrm{HNMR}$ Spectrum of $( \pm)-7$

${ }^{13} \mathrm{C}\left\{{ }^{1} \mathrm{H}\right\}$ NMR Spectrum of $( \pm)-7$

${ }^{1} \mathrm{H}$ NMR Spectrum of (-)-7

${ }^{13} \mathrm{C}\left\{{ }^{1} \mathrm{H}\right\}$ NMR Spectrum of $(-)-7$

${ }^{1} \mathrm{H}$ NMR Spectrum of $(+)-7$

${ }^{13} \mathrm{C}\left\{{ }^{1} \mathrm{H}\right\}$ NMR Spectrum of $(+)-7$

${ }^{1} \mathrm{H}$ NMR Spectrum of $( \pm)-8$

${ }^{13} \mathrm{C}\left\{{ }^{1} \mathrm{H}\right\}$ NMR Spectrum of $( \pm)-8$

${ }^{1} \mathrm{H}$ NMR Spectrum of (-)-8

${ }^{13} \mathrm{C}\left\{{ }^{1} \mathrm{H}\right\}$ NMR Spectrum of (-)-8

${ }^{1} \mathrm{H}$ NMR Spectrum of $(+)-8$

${ }^{13} \mathrm{C}\left\{{ }^{1} \mathrm{H}\right\}$ NMR Spectrum of $(+)-8$

${ }^{1} \mathrm{H}$ NMR Spectrum of $( \pm)-\mathbf{4}$

${ }^{13} \mathrm{C}\left\{{ }^{1} \mathrm{H}\right\}$ NMR Spectrum of $( \pm)-4$

${ }^{1} \mathrm{H}$ NMR Spectrum of (+)-4 $\left(126 \mathrm{MHz} \mathrm{CDCl}_{3}\right.$ )

$\mathrm{SI}-14$

$\left(500 \mathrm{MHz}, \mathrm{CDCl}_{3}\right)$

$\left(126 \mathrm{MHz}, \mathrm{CDCl}_{3}\right.$ )

$\left(500 \mathrm{MHz}, \mathrm{CDCl}_{3}\right.$ )

(126 MHz, $\mathrm{CDCl}_{3}$ )

(500 MHz, $\mathrm{CDCl}_{3}$ )

(126 MHz, $\mathrm{CDCl}_{3}$ )

(500 MHz, $\mathrm{CDCl}_{3}$ )

(126 MHz, $\mathrm{CDCl}_{3}$ )

(500 MHz, $\mathrm{CDCl}_{3}$ )

$\left(126 \mathrm{MHz}, \mathrm{CDCl}_{3}\right.$ )

$\left(500 \mathrm{MHz}, \mathrm{CDCl}_{3}\right.$ )

(126 MHz, $\mathrm{CDCl}_{3}$ )

$\left(500 \mathrm{MHz}, \mathrm{CDCl}_{3}\right.$ )

(126 MHz, $\mathrm{CDCl}_{3}$ )

$\left(500 \mathrm{MHz}, \mathrm{CDCl}_{3}\right.$ )

(126 MHz, $\mathrm{CDCl}_{3}$ )

(500 MHz, $\mathrm{CDCl}_{3}$ )

(126 MHz, $\mathrm{CDCl}_{3}$ )

(500 MHz, $\mathrm{CDCl}_{3}$ )

(126 MHz, $\mathrm{CDCl}_{3}$ )

$\left(500 \mathrm{MHz}, \mathrm{CDCl}_{3}\right.$ )

(126 MHz, $\mathrm{CDCl}_{3}$ )

(500 MHz, $\mathrm{CDCl}_{3}$ )
SI-15

SI-16

SI-17

SI-18

SI-19

SI-20

SI-21

SI-22

$\mathrm{SI}-23$

SI-24

SI-25

SI-26

SI-27

SI-28

SI-29

SI-30

SI-31

SI-32

SI-33

SI-34

SI-35

SI-36

SI-37 
${ }^{13} \mathrm{C}\left\{{ }^{1} \mathrm{H}\right\}$ NMR Spectrum of $(+)-4 \quad\left(126 \mathrm{MHz}, \mathrm{CDCl}_{3}\right)$

SI-38

${ }^{1} \mathrm{H}$ NMR Spectrum of $(-)-4$

$\left(500 \mathrm{MHz}, \mathrm{CDCl}_{3}\right)$

SI-39

${ }^{13} \mathrm{C}\left\{{ }^{1} \mathrm{H}\right\}$ NMR Spectrum of $(-)-4 \quad\left(126 \mathrm{MHz}, \mathrm{CDCl}_{3}\right)$

SI-40

${ }^{1} \mathrm{H}$ NMR Spectrum of $(-)-9$

$\left(400 \mathrm{MHz}, \mathrm{CDCl}_{3}\right)$

SI-41

${ }^{13} \mathrm{C}\left\{{ }^{1} \mathrm{H}\right\}$ NMR Spectrum of $(-)-9$

(101 $\mathrm{MHz}, \mathrm{CDCl}_{3}$ )

SI-42

${ }^{1} \mathrm{H}$ NMR Spectrum of $(+)-10$

$\left(500 \mathrm{MHz}, \mathrm{CDCl}_{3}\right)$

SI-43

${ }^{13} \mathrm{C}\left\{{ }^{1} \mathrm{H}\right\}$ NMR Spectrum of $(+)-10$

(126 $\left.\mathrm{MHz}, \mathrm{CDCl}_{3}\right)$

SI-44

${ }^{1} \mathrm{H}$ NMR Spectrum of $(-)-\mathbf{1 1}$

$\left(400 \mathrm{MHz}, \mathrm{CDCl}_{3}\right)$

SI-45

${ }^{13} \mathrm{C}\left\{{ }^{1} \mathrm{H}\right\}$ NMR Spectrum of $(-)-11 \quad\left(101 \mathrm{MHz}, \mathrm{CDCl}_{3}\right)$

SI-46

${ }^{1} \mathrm{H}$ NMR Spectrum of $(+)-12$

$\left(500 \mathrm{MHz}, \mathrm{CDCl}_{3}\right)$

SI-47

${ }^{13} \mathrm{C}\left\{{ }^{1} \mathrm{H}\right\}$ NMR Spectrum of $(+)-12$

(126 $\mathrm{MHz}, \mathrm{CDCl}_{3}$ )

SI-48

${ }^{1} \mathrm{H}$ NMR Spectrum of $\mathbf{S 1}$

$\left(500 \mathrm{MHz}, \mathrm{CDCl}_{3}\right.$ )

SI-49

${ }^{13} \mathrm{C}\left\{{ }^{1} \mathrm{H}\right\}$ NMR Spectrum of $\mathbf{S 1}$

(126 MHz, $\mathrm{CDCl}_{3}$ )

SI-50

${ }^{1} \mathrm{H}$ NMR Spectrum of $\mathbf{S 2}$

(500 MHz, $\mathrm{CDCl}_{3}$ )

SI-51

${ }^{13} \mathrm{C}\left\{{ }^{1} \mathrm{H}\right\}$ NMR Spectrum of $\mathbf{S 2}$

(126 MHz, $\mathrm{CDCl}_{3}$ )

SI-52

${ }^{1} \mathrm{HNMR}$ Spectrum of $\mathbf{S 3}$

$\left(500 \mathrm{MHz}, \mathrm{CDCl}_{3}\right)$

SI-53

${ }^{13} \mathrm{C}\left\{{ }^{1} \mathrm{H}\right\}$ NMR Spectrum of S3

(126 MHz, $\mathrm{CDCl}_{3}$ )

SI-54

${ }^{1} \mathrm{H}$ NMR Spectrum of 13

(500 MHz, $\mathrm{CDCl}_{3}$ )

SI-55

${ }^{13} \mathrm{C}\left\{{ }^{1} \mathrm{H}\right\}$ NMR Spectrum of 13

(126 MHz, $\mathrm{CDCl}_{3}$ )

SI-56

${ }^{1} \mathrm{H}$ NMR Spectrum of $\mathbf{1 4 a}$

(500 MHz, $\mathrm{CD}_{2} \mathrm{Cl}_{2}$ )

SI-57

${ }^{13} \mathrm{C}\left\{{ }^{1} \mathrm{H}\right\}$ NMR Spectrum of $14 \mathrm{a}$

(126 MHz, $\mathrm{CD}_{2} \mathrm{Cl}_{2}$ )

SI-58

${ }^{1} \mathrm{H}$ NMR Spectrum of $\mathbf{1 4 b}$

(400 MHz, $\mathrm{CDCl}_{3}$ )

SI-59

${ }^{13} \mathrm{C}\left\{{ }^{1} \mathrm{H}\right\}$ NMR Spectrum of $\mathbf{1 4 b}$

(101 MHz, $\mathrm{CD}_{2} \mathrm{Cl}_{2}$ )

SI-60

${ }^{1} \mathrm{H}$ NMR Spectrum of $(1 R, 2 S)-2-\{[$ Bis(tert-butoxycarbonyl)amino]methyl\}cyclopropyl- $N$ -

$\left\{(1 S)\right.$-2-(dimethyl-amino)-1-[(2-methyl)propyl]-2-oxoethyl\}acetamide (400 MHz, $\left.\mathrm{CDCl}_{3}\right) \quad$ SI-61 ${ }^{13} \mathrm{C}\left\{{ }^{1} \mathrm{H}\right\}$ NMR Spectrum of $(1 R, 2 S)-2-\{[$ Bis (tert-butoxycarbonyl)amino]methyl\}cyclopropyl- $N$ -

$\left\{(1 S)\right.$-2-(dimethyl-amino)-1-[(2-methyl)propyl]-2-oxoethyl\}acetamide (101 MHz, $\left.\mathrm{CDCl}_{3}\right)$ SI-62

${ }^{1} \mathrm{H}$ NMR Spectrum of $16 \mathbf{a}$

$\left(500 \mathrm{MHz}, \mathrm{CD}_{2} \mathrm{Cl}_{2}\right)$

SI-63

${ }^{13} \mathrm{C}\left\{{ }^{1} \mathrm{H}\right\}$ NMR Spectrum of $16 \mathbf{a}$

(126 MHz, $\mathrm{CD}_{2} \mathrm{Cl}_{2}$ )

SI-64

${ }^{1} \mathrm{H}$ NMR Spectrum of $(1 S, 2 R)-2-\{[\mathrm{Bis}($ tert-butoxycarbonyl)amino]methyl\}cyclopropyl- $N$ -

$\left\{(1 S)\right.$-2-(dimethyl-amino)-1-[(2-methyl)propyl]-2-oxoethyl\}acetamide (500 MHz, $\left.\mathrm{CDCl}_{3}\right)$ SI-65

${ }^{13} \mathrm{C}\left\{{ }^{1} \mathrm{H}\right\}$ NMR Spectrum of $(1 S, 2 R)-2-\{[$ Bis (tert-butoxycarbonyl)amino]methyl $\}$ cyclopropyl- $N$ -

$\left\{(1 S)\right.$-2-(dimethyl-amino)-1-[(2-methyl)propyl]-2-oxoethyl\}acetamide (126 MHz, $\mathrm{CDCl}_{3}$ ) SI-66

${ }^{1} \mathrm{H}$ NMR Spectrum of $\mathbf{1 6 b}$

$\left(500 \mathrm{MHz}, \mathrm{CDCl}_{3}\right)$

SI-67

${ }^{13} \mathrm{C}\left\{{ }^{1} \mathrm{H}\right\}$ NMR Spectrum of $16 \mathrm{~b}$

$\left(126 \mathrm{MHz}, \mathrm{CDCl}_{3}\right)$

SI-68

${ }^{1} \mathrm{H}$ NMR Spectrum of $\left(1 S^{*}, 2 R^{*}\right)-2-\{2-[\mathrm{Bis}($ tert-butoxycarbonyl)amino]methyl\}-

cyclopropyl- $N$-[2-(dimethylamino)-1-oxoethyl]acetamide $\left(500 \mathrm{MHz}, \mathrm{CDCl}_{3}\right.$ )

SI-69 
${ }^{13} \mathrm{C}\left\{{ }^{1} \mathrm{H}\right\}$ NMR Spectrum of $\left(1 \mathrm{~S}^{*}, 2 R^{*}\right)-2-\{2-[$ Bis (tert-butoxycarbonyl)amino]methyl\}cyclopropyl- $N$-[2-(dimethylamino)-1-oxoethyl]acetamide $\left(126 \mathrm{MHz} \mathrm{CDCl}_{3}\right) \quad \mathrm{SI}$-70 ${ }^{1} \mathrm{H}$ NMR Spectrum of $( \pm)-17 \quad\left(400 \mathrm{MHz}^{\left.-17 \mathrm{CDCl}_{3}\right)} \quad\right.$ SI-71

${ }^{13} \mathrm{C}\left\{{ }^{1} \mathrm{H}\right\}$ NMR Spectrum of $( \pm)-17 \quad\left(101 \mathrm{MHz}, \mathrm{CDCl}_{3}\right) \quad$ SI-72

${ }^{1} \mathrm{H}-{ }^{1} \mathrm{H}$ NOESY Spectrum of $16 \mathrm{a} \quad\left(600 \mathrm{MHz}, \mathrm{CD}_{2} \mathrm{Cl}_{2}\right) \quad$ SI-73

${ }^{1} \mathrm{H}-{ }^{1} \mathrm{H}$ NOESY Spectrum of $\mathbf{1 6 b} \quad\left(600 \mathrm{MHz}, \mathrm{CD}_{2} \mathrm{Cl}_{2}\right) \quad$ SI-74 
$\mathrm{HO}^{\prime \prime \prime}$

$( \pm)-5$

${ }^{1} \mathrm{H}$ NMR $\left(400 \mathrm{MHz}, \mathrm{CDCl}_{3}\right)$

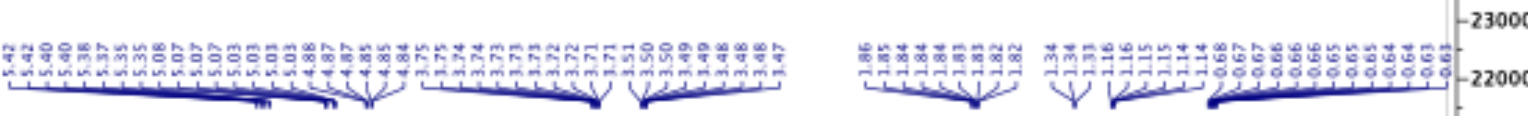
10

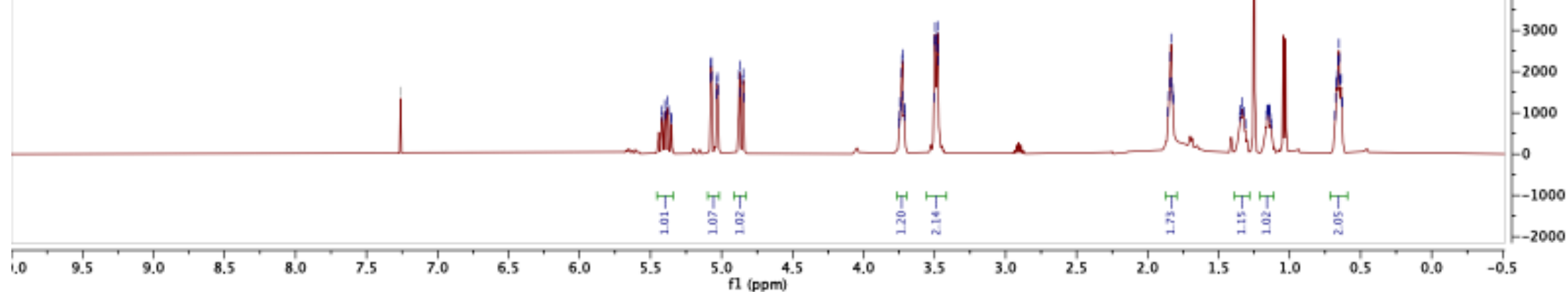


$\overbrace{( \pm)-5}^{\text {HO' }}$

${ }^{13} \mathrm{C}\left\{{ }^{1} \mathrm{H}\right\}$ NMR $\left(101 \mathrm{MHz}, \mathrm{CDCl}_{3}\right)$

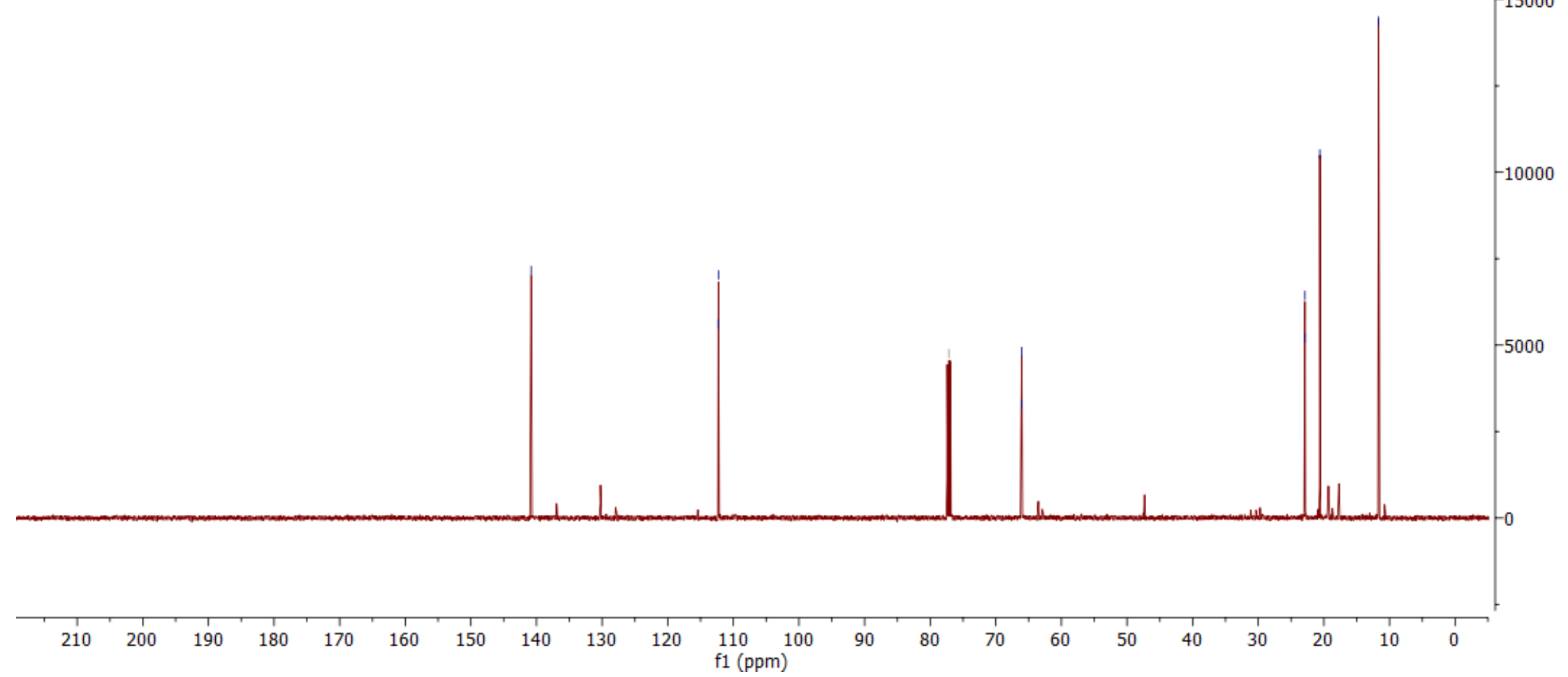




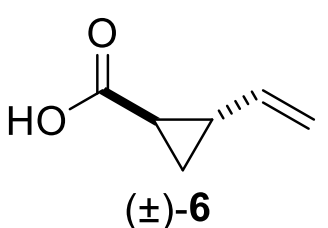

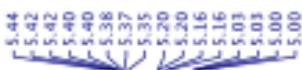

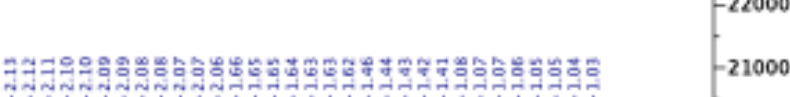

$\pm)-6$

${ }^{1} \mathrm{H}$ NMR $\left(400 \mathrm{MHz}, \mathrm{CDCl}_{3}\right)$
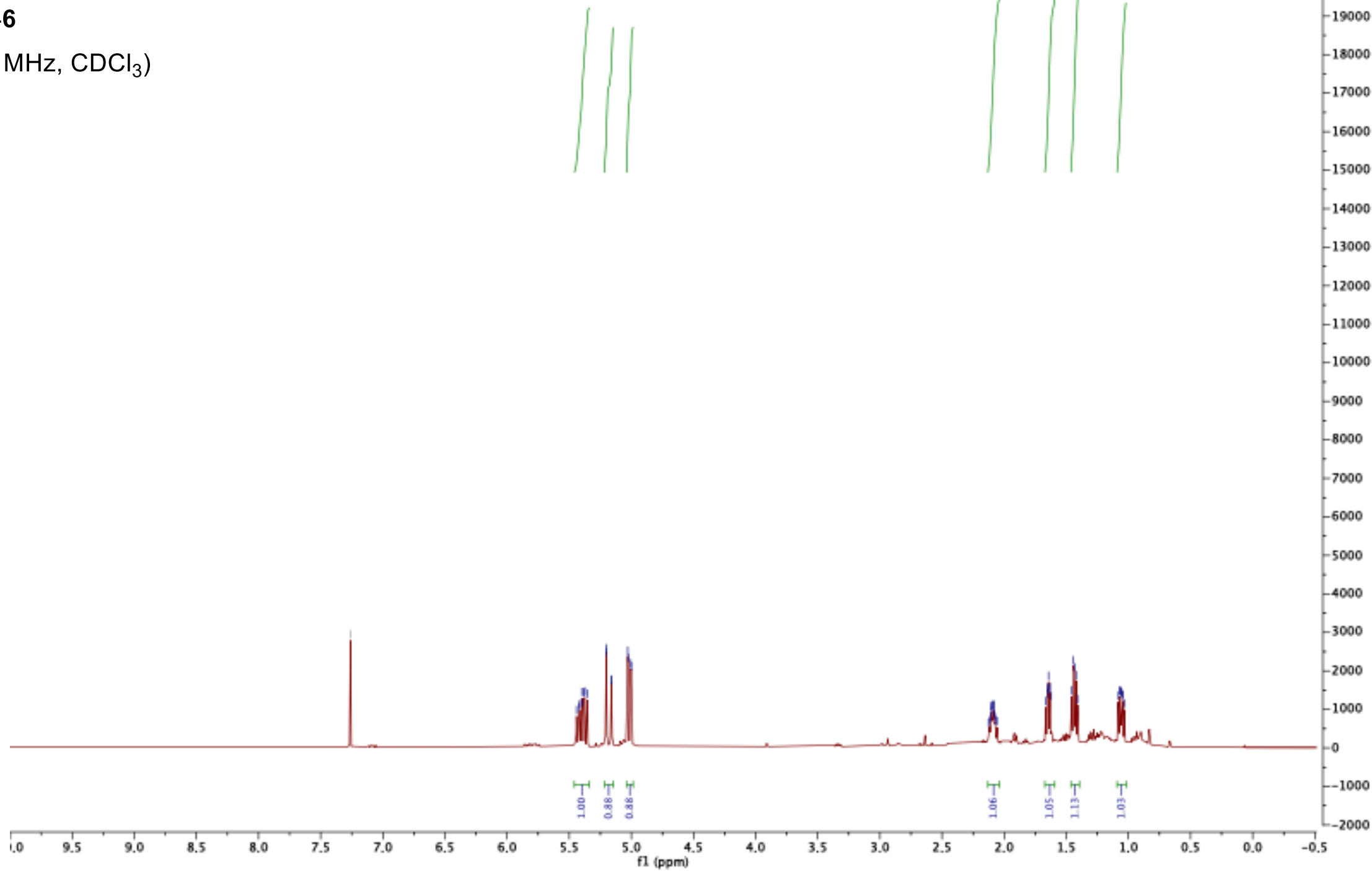
<smiles>C=C[C@H]1CC1C(=O)O</smiles>

(士)-6

${ }^{13} \mathrm{C}\left\{{ }^{1} \mathrm{H}\right\}$ NMR $\left(126 \mathrm{MHz}, \mathrm{CDCl}_{3}\right)$

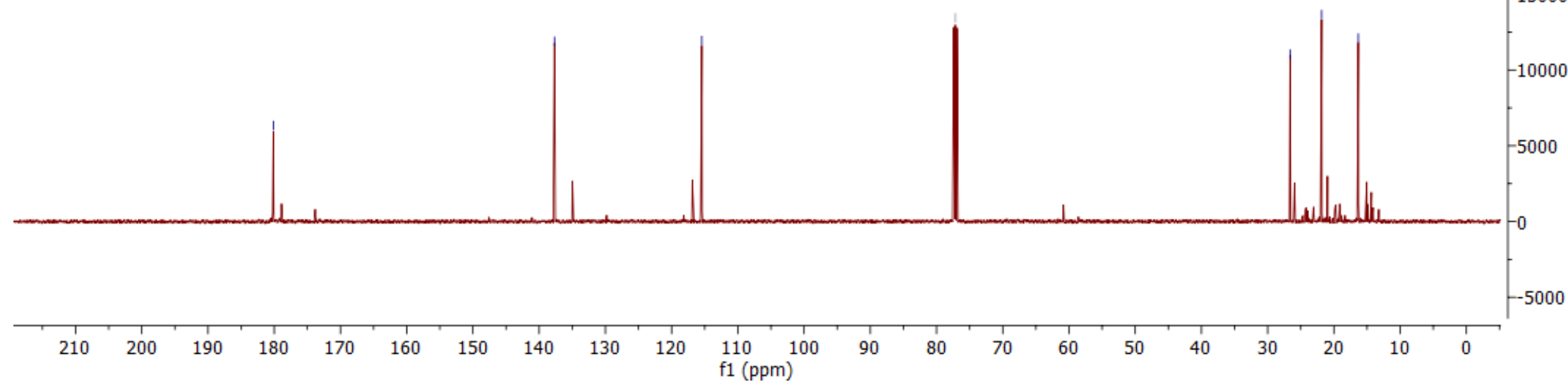




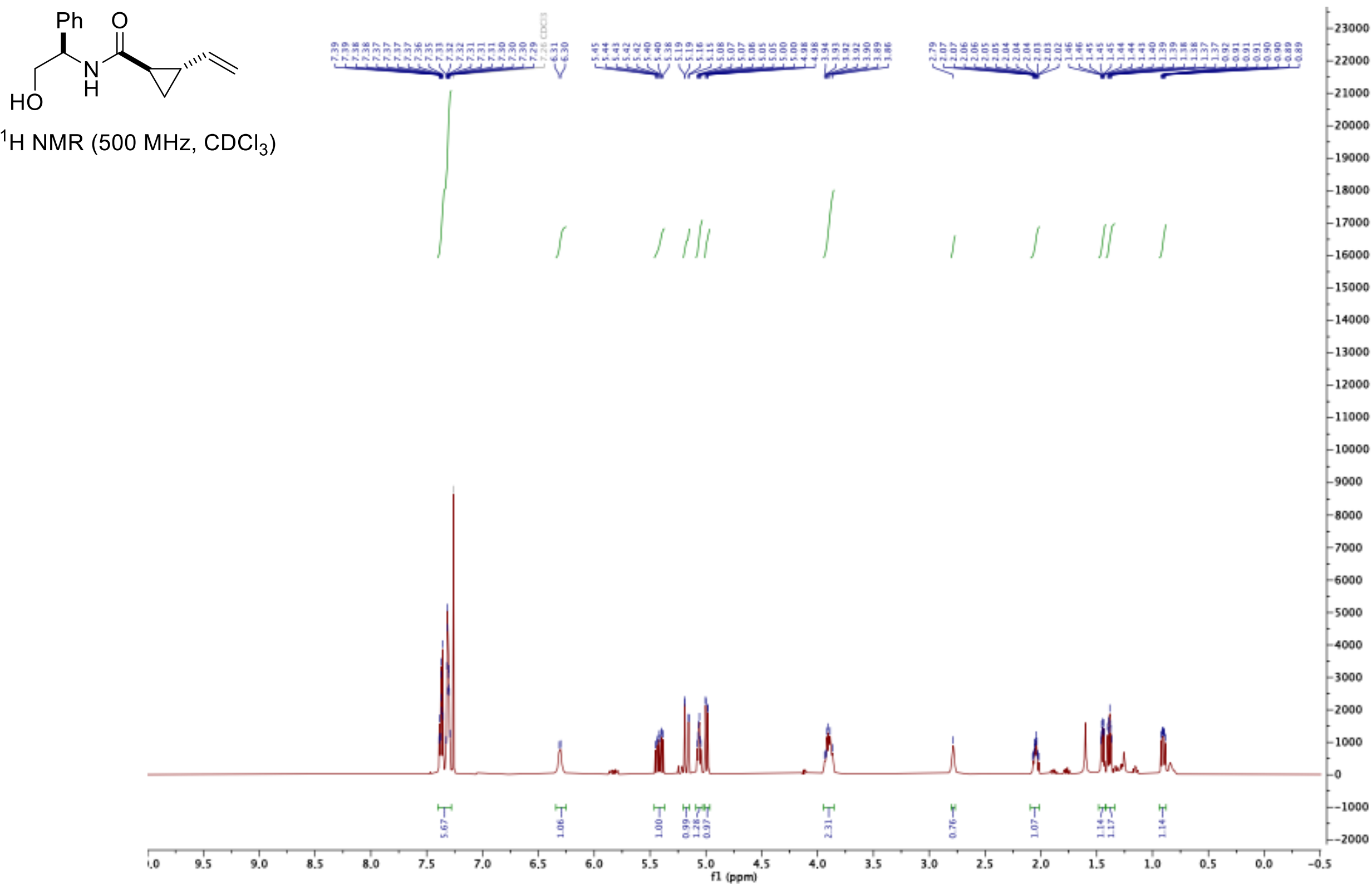

SI-11 


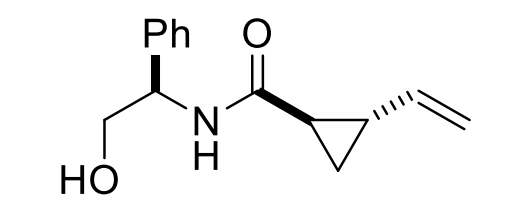

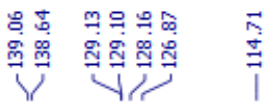

${ }^{13} \mathrm{C}\left\{{ }^{1} \mathrm{H}\right\}$ NMR $\left(126 \mathrm{MHz}, \mathrm{CDCl}_{3}\right)$
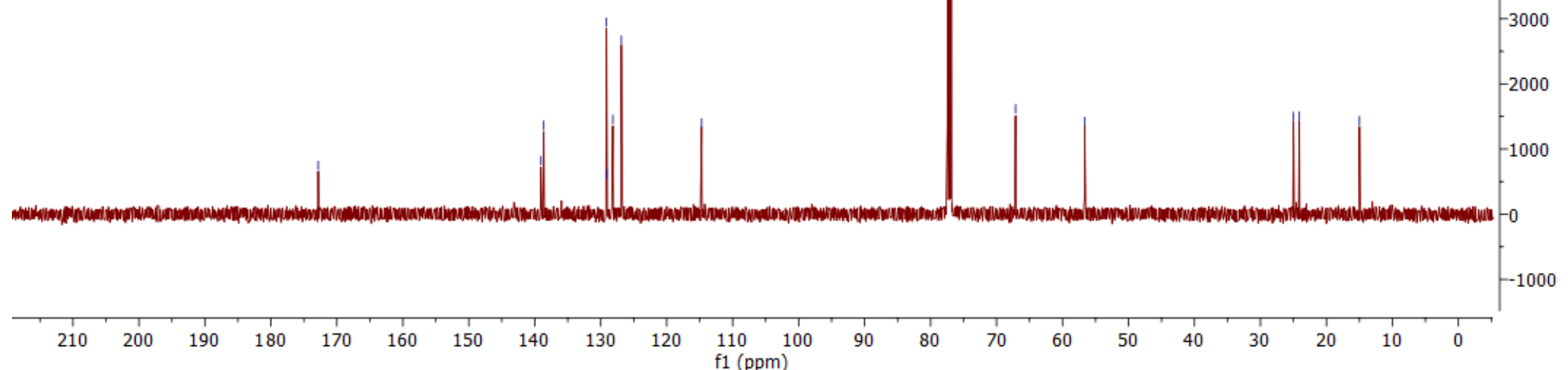

SI-12 


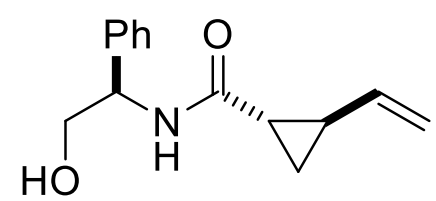

${ }^{1} \mathrm{H}$ NMR $\left(500 \mathrm{MHz}, \mathrm{CDCl}_{3}\right)$
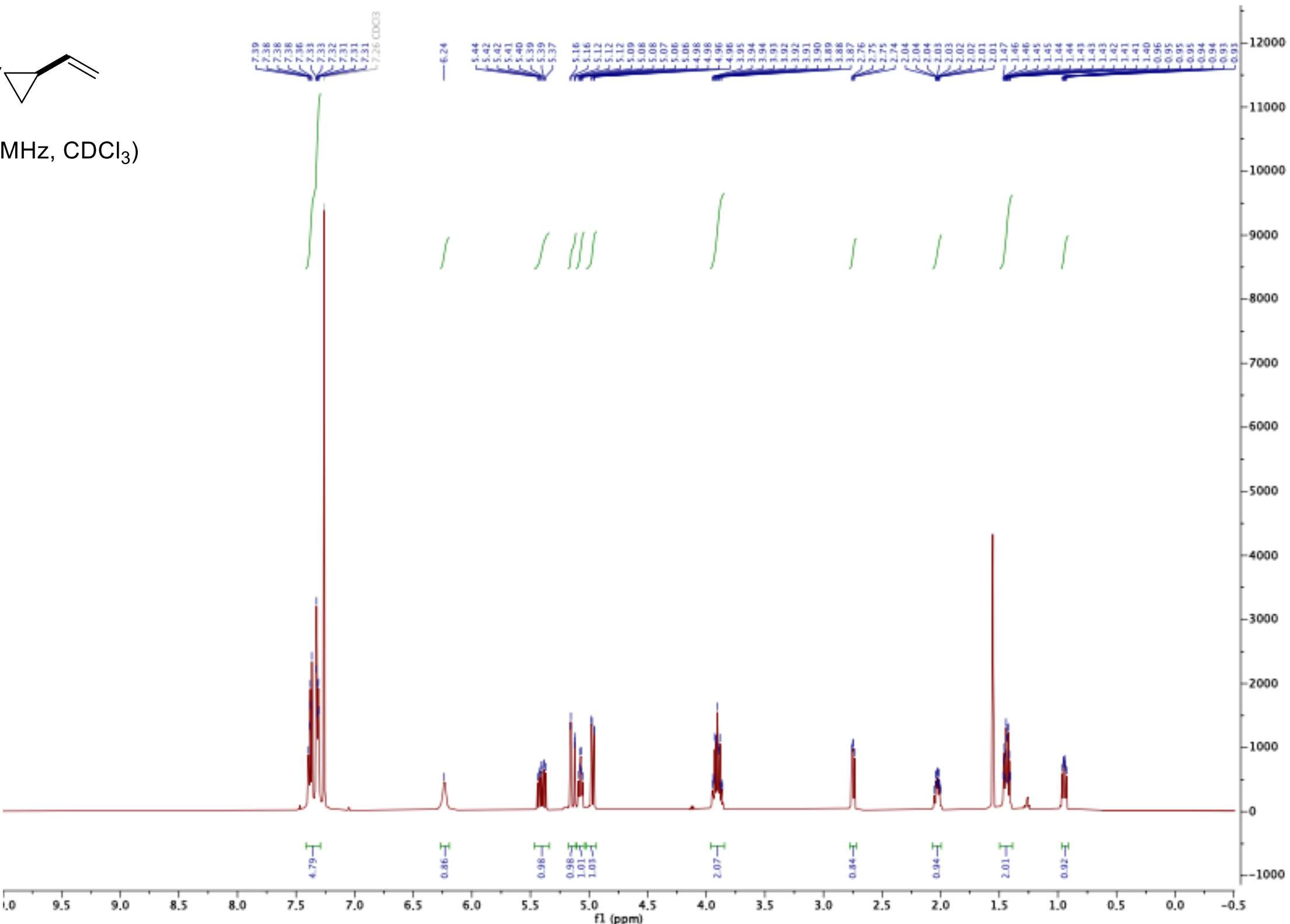

SI-13 
<smiles>C=C[C@@H]1C[C@H]1C(=O)NC(CO)c1ccccc1</smiles>

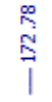

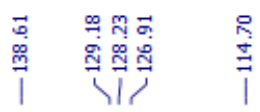

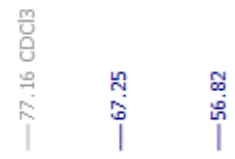

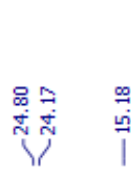

${ }^{13} \mathrm{C}\left\{{ }^{1} \mathrm{H}\right\}$ NMR $\left(126 \mathrm{MHz}, \mathrm{CDCl}_{3}\right)$

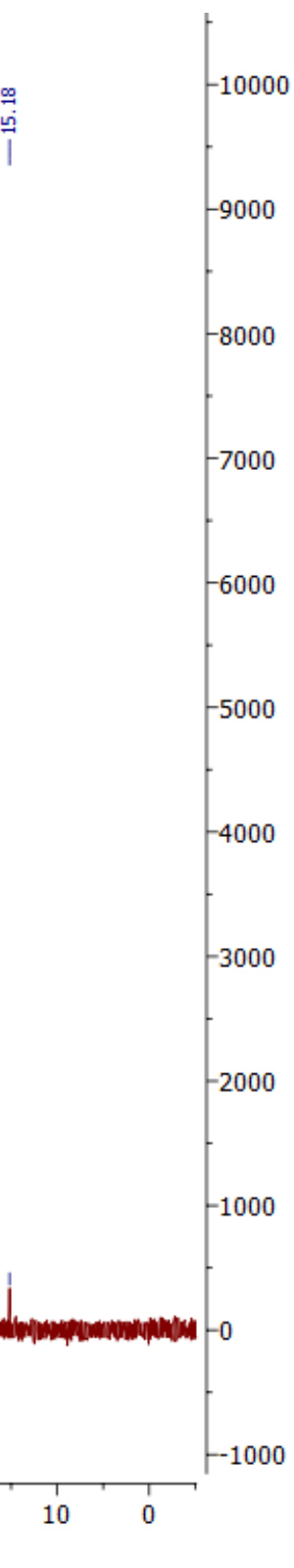

SI-14 


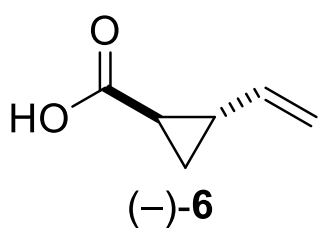

\section{๑ง}

${ }^{1} \mathrm{H}$ NMR $\left(500 \mathrm{MHz}, \mathrm{CDCl}_{3}\right)$

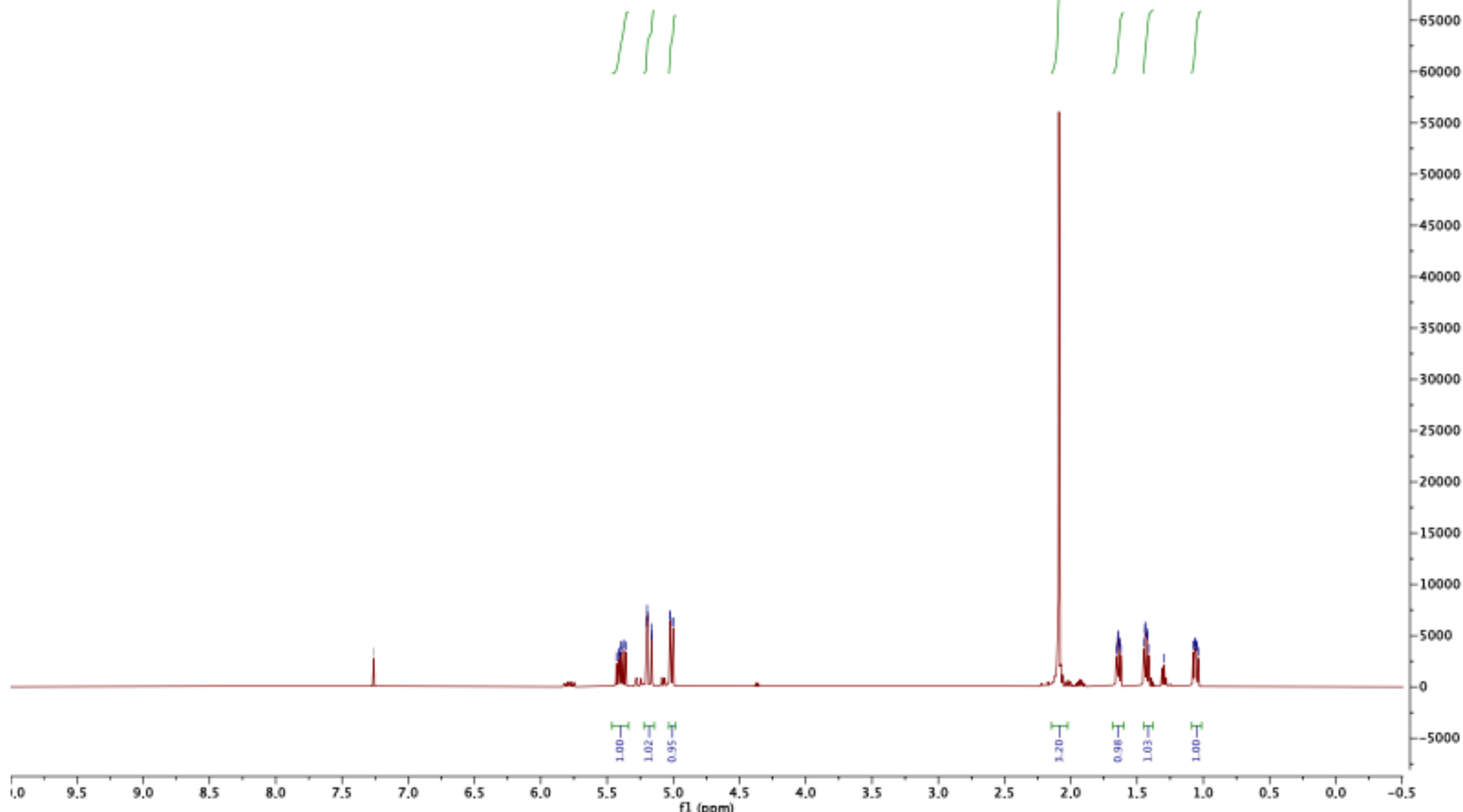

SI-15 

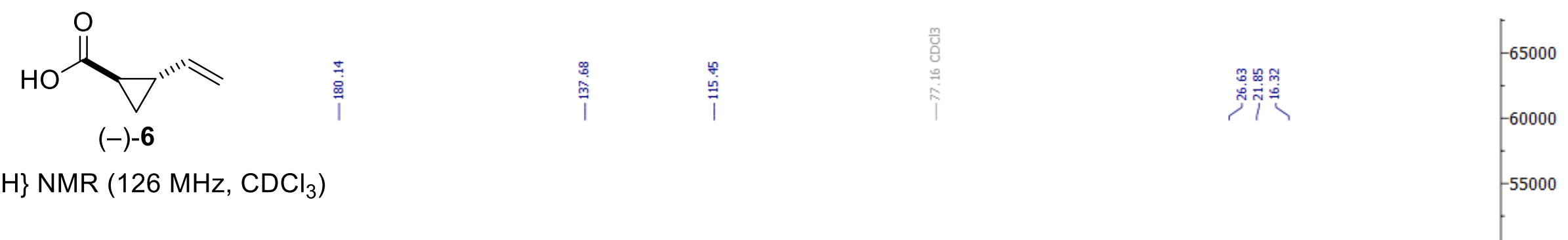

${ }^{13} \mathrm{C}\left\{{ }^{1} \mathrm{H}\right\} \mathrm{NMR}\left(126 \mathrm{MHz}, \mathrm{CDCl}_{3}\right)$
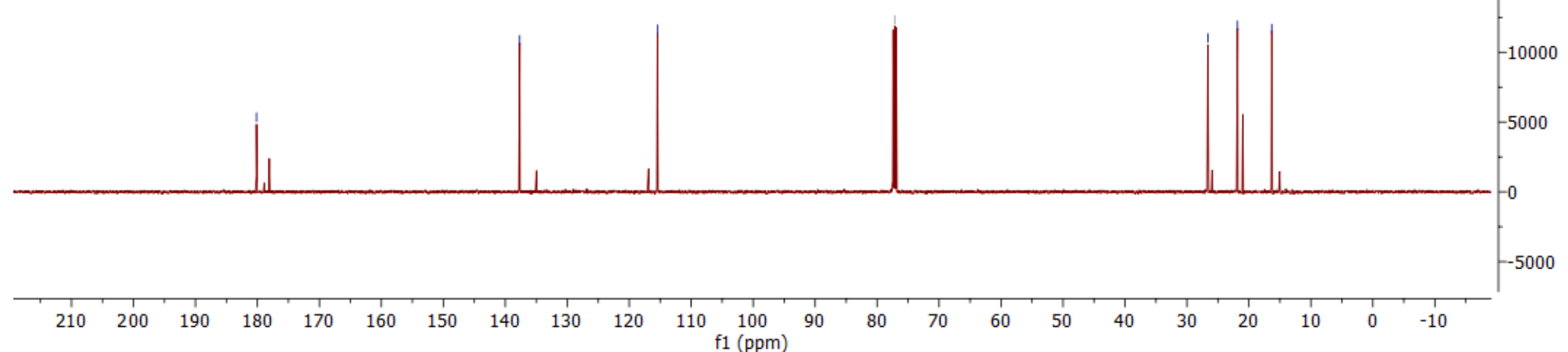

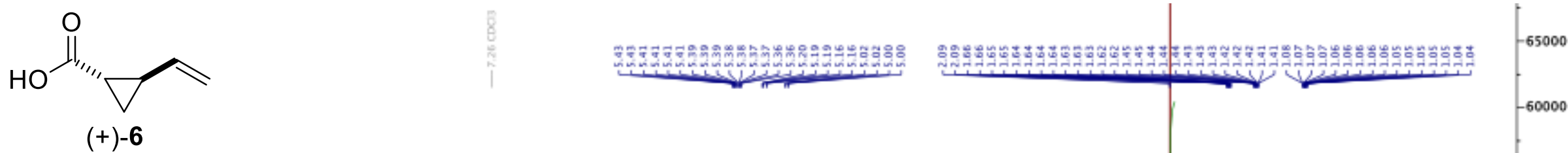

${ }^{1} \mathrm{H}$ NMR $\left(500 \mathrm{MHz}, \mathrm{CDCl}_{3}\right)$

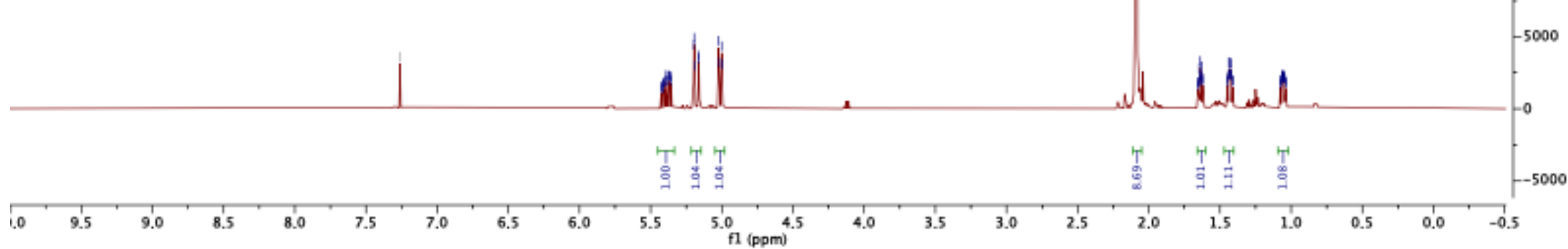

SI-17 

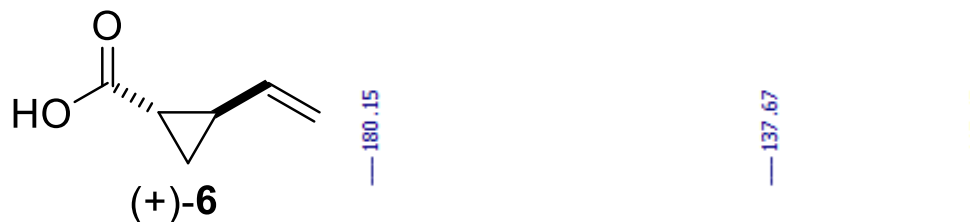

i

$\frac{m}{0}$
0
0
0

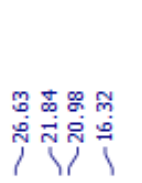

$-28000$

$-26000$

24000

${ }^{13} \mathrm{C}\left\{{ }^{1} \mathrm{H}\right\} \operatorname{NMR}\left(126 \mathrm{MHz}, \mathrm{CDCl}_{3}\right)$ 
$\underbrace{\text { HO }}_{(-)-5}{ }^{\prime \prime \prime}$

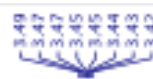

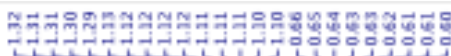

$-40000$

${ }^{1} \mathrm{H}$ NMR $\left(500 \mathrm{MHz}, \mathrm{CDCl}_{3}\right)$

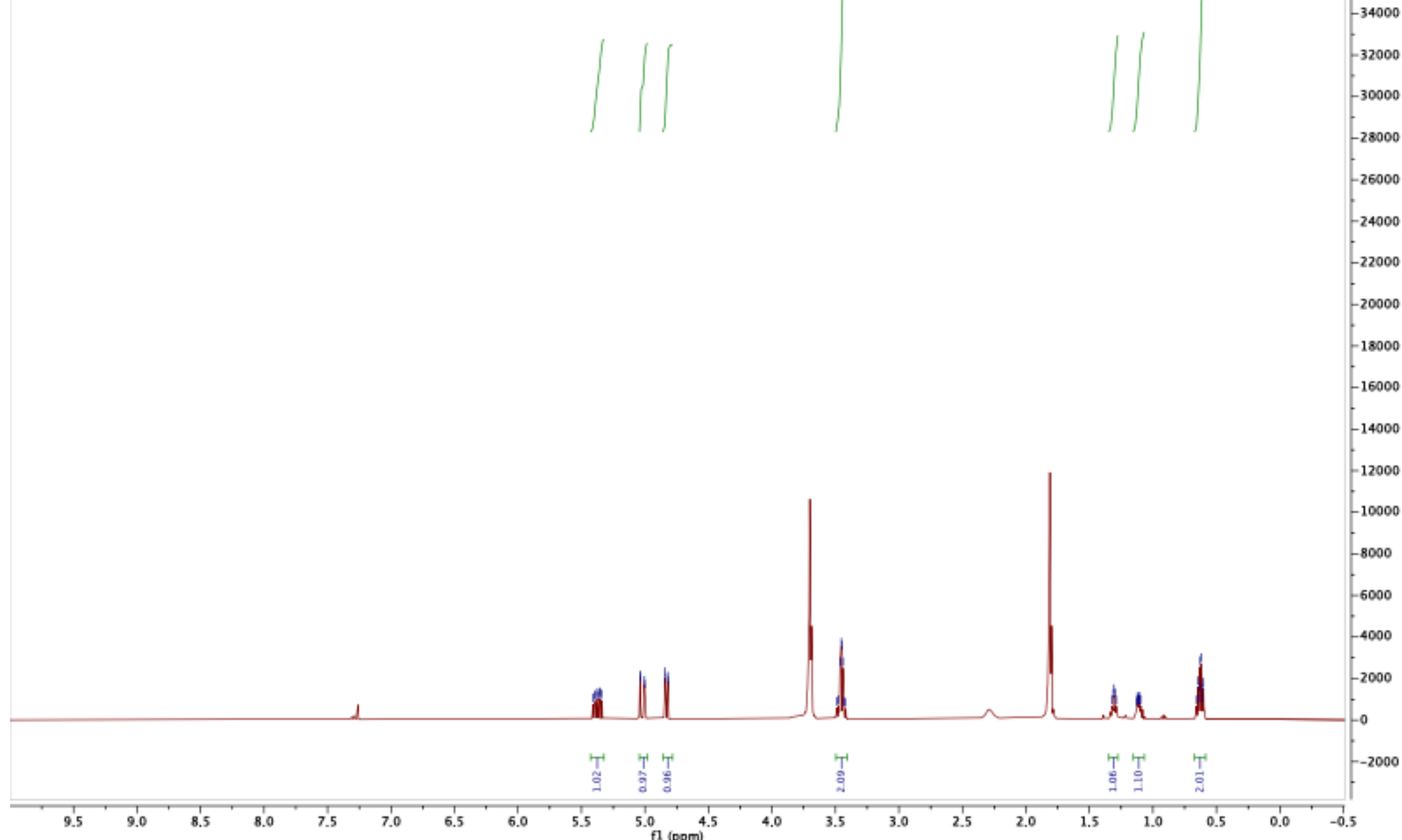

SI-19 
$\mathrm{VO}_{(-)-5}^{\text {HO' }}$

高

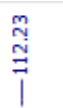

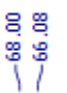

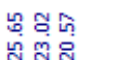

${ }^{13} \mathrm{C}\left\{{ }^{1} \mathrm{H}\right\}$ NMR $\left(126 \mathrm{MHz}, \mathrm{CDCl}_{3}\right)$

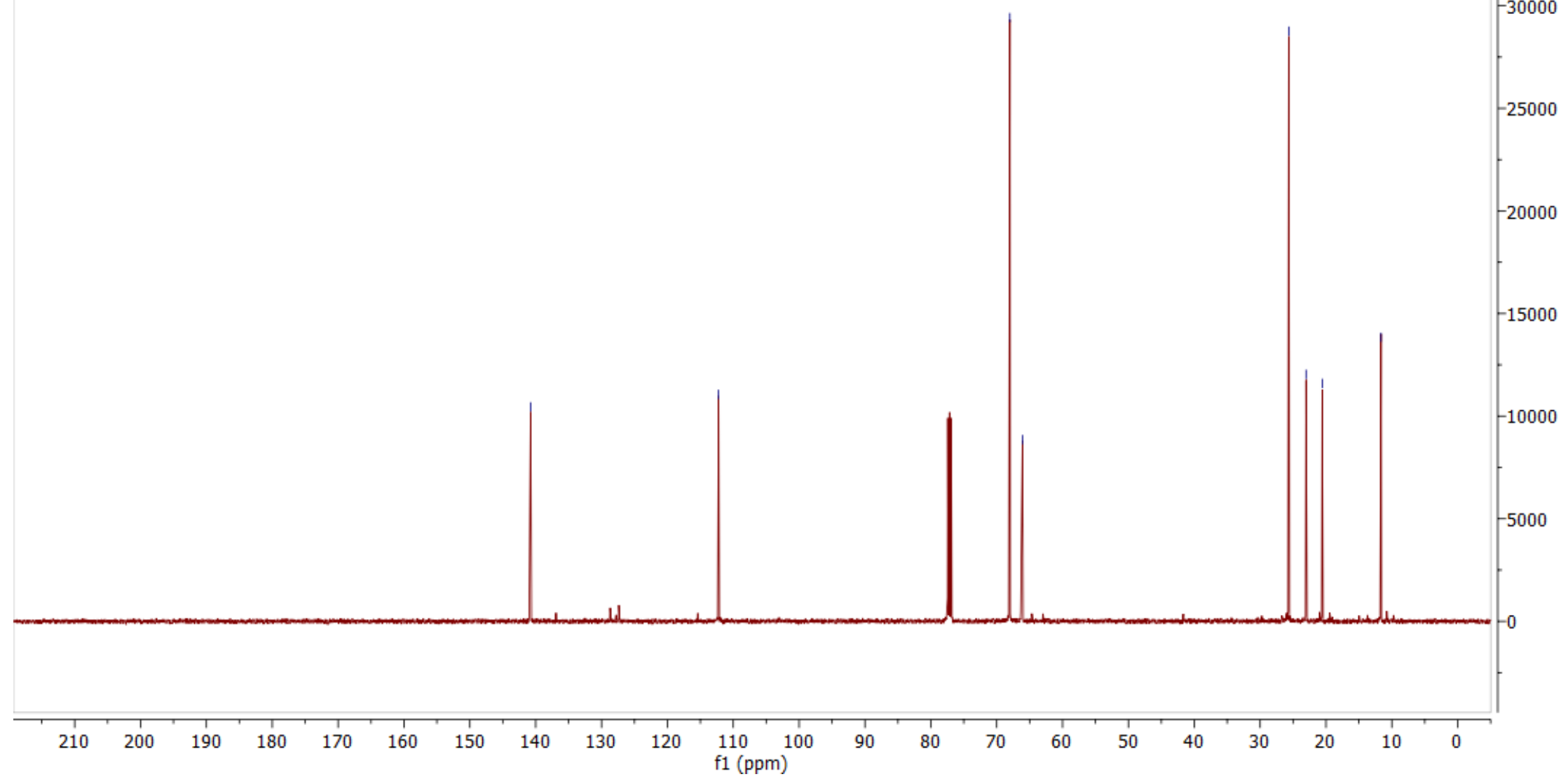


$\mathrm{HO}^{-\prime \prime \prime \prime}$

(+)-5

${ }^{1} \mathrm{H}$ NMR $\left(500 \mathrm{MHz}, \mathrm{CDCl}_{3}\right)$

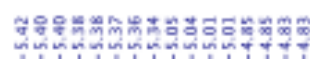

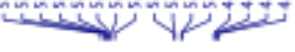

锩哿

ำกำ

30000
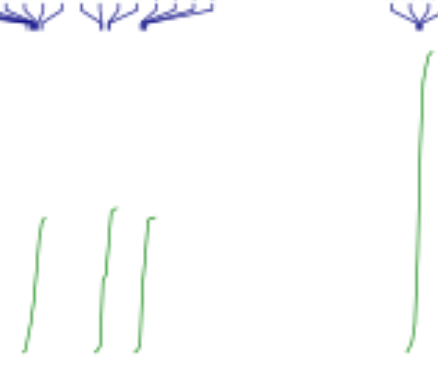

20000

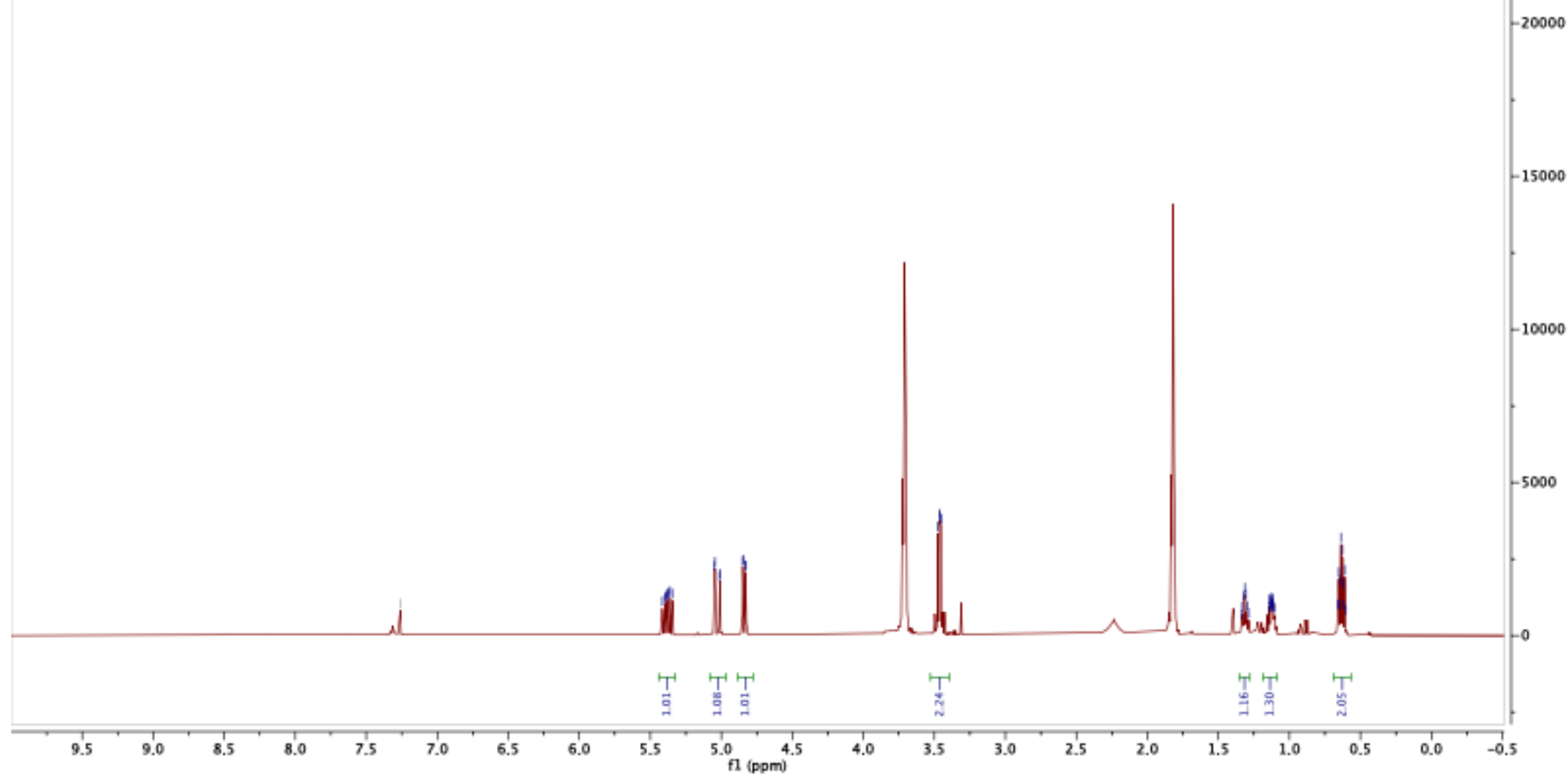

SI-21 

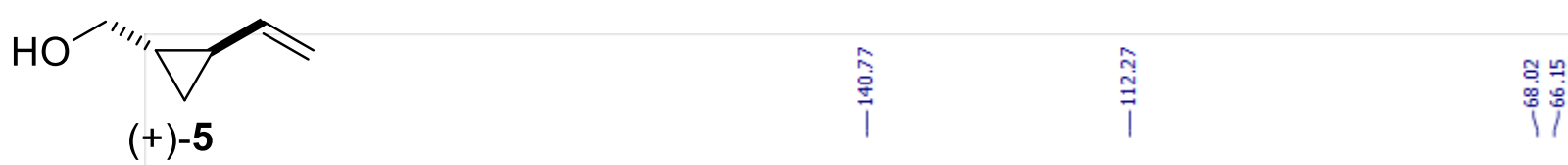

象

${ }^{13} \mathrm{C}\left\{{ }^{1} \mathrm{H}\right\}$ NMR $\left(126 \mathrm{MHz}, \mathrm{CDCl}_{3}\right)$

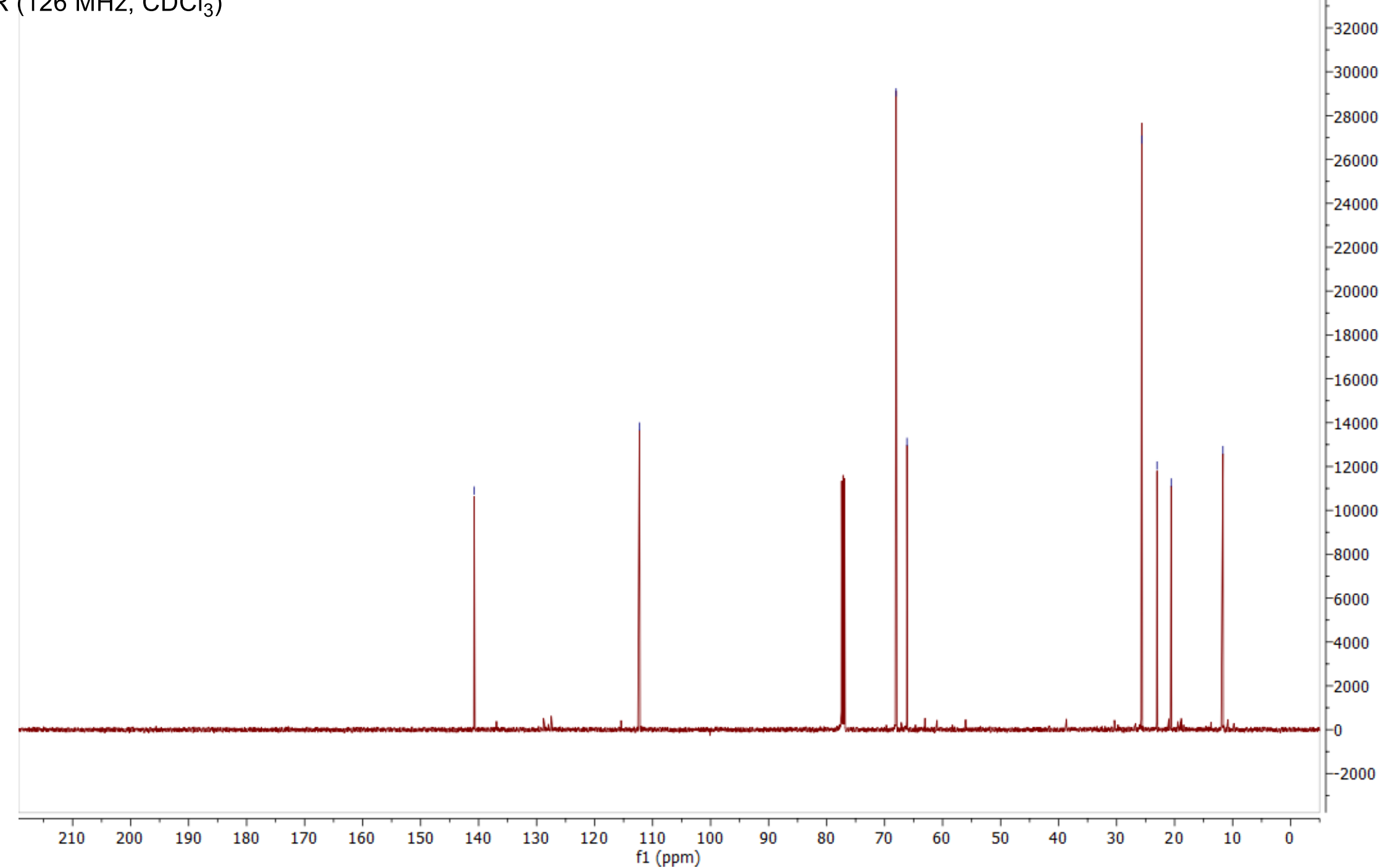


$(\mathrm{Boc})_{2}$

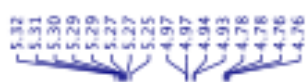

$( \pm)-7$

${ }^{1} \mathrm{H}$ NMR $\left(500 \mathrm{MHz}, \mathrm{CDCl}_{3}\right)$

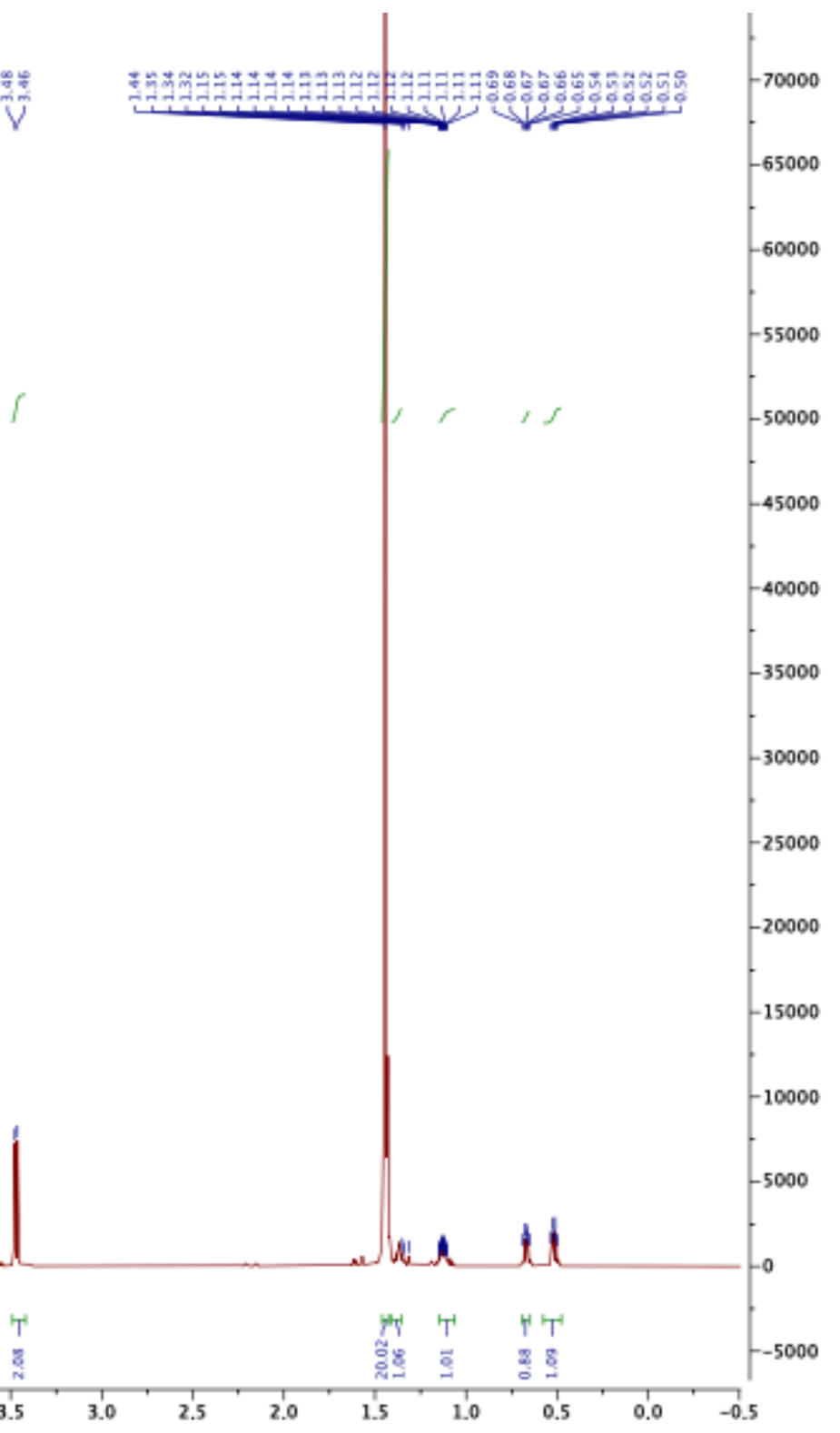

SI-23 
$(\mathrm{Boc})_{2} \mathrm{~N} \nabla^{\prime \prime \prime}$

$( \pm)-7$

${ }^{13} \mathrm{C}\left\{{ }^{1} \mathrm{H}\right\}$ NMR $\left(126 \mathrm{MHz}, \mathrm{CDCl}_{3}\right)$

I

覚

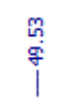

$-55000$

$-50000$

$-45000$

40000

$-35000$

30000

$-25000$

$-20000$

$-15000$

10000

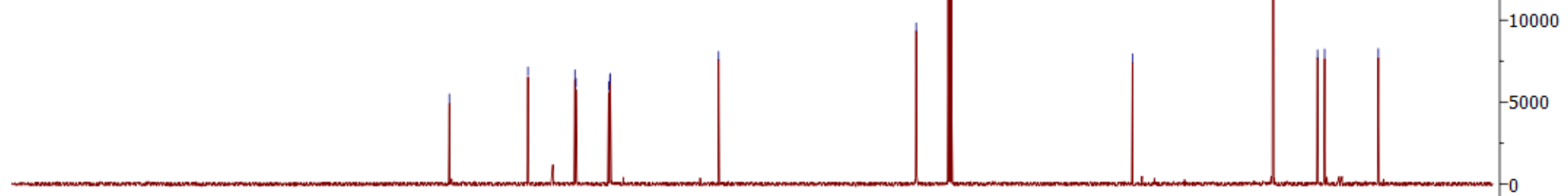

SI-24 


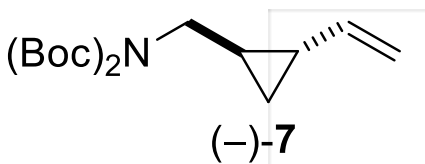

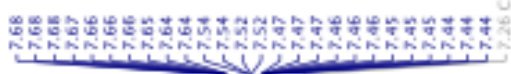

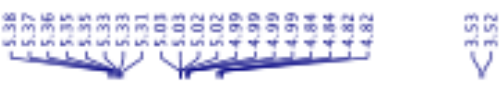

${ }^{1} \mathrm{H}$ NMR $\left(500 \mathrm{MHz}, \mathrm{CDCl}_{3}\right)$

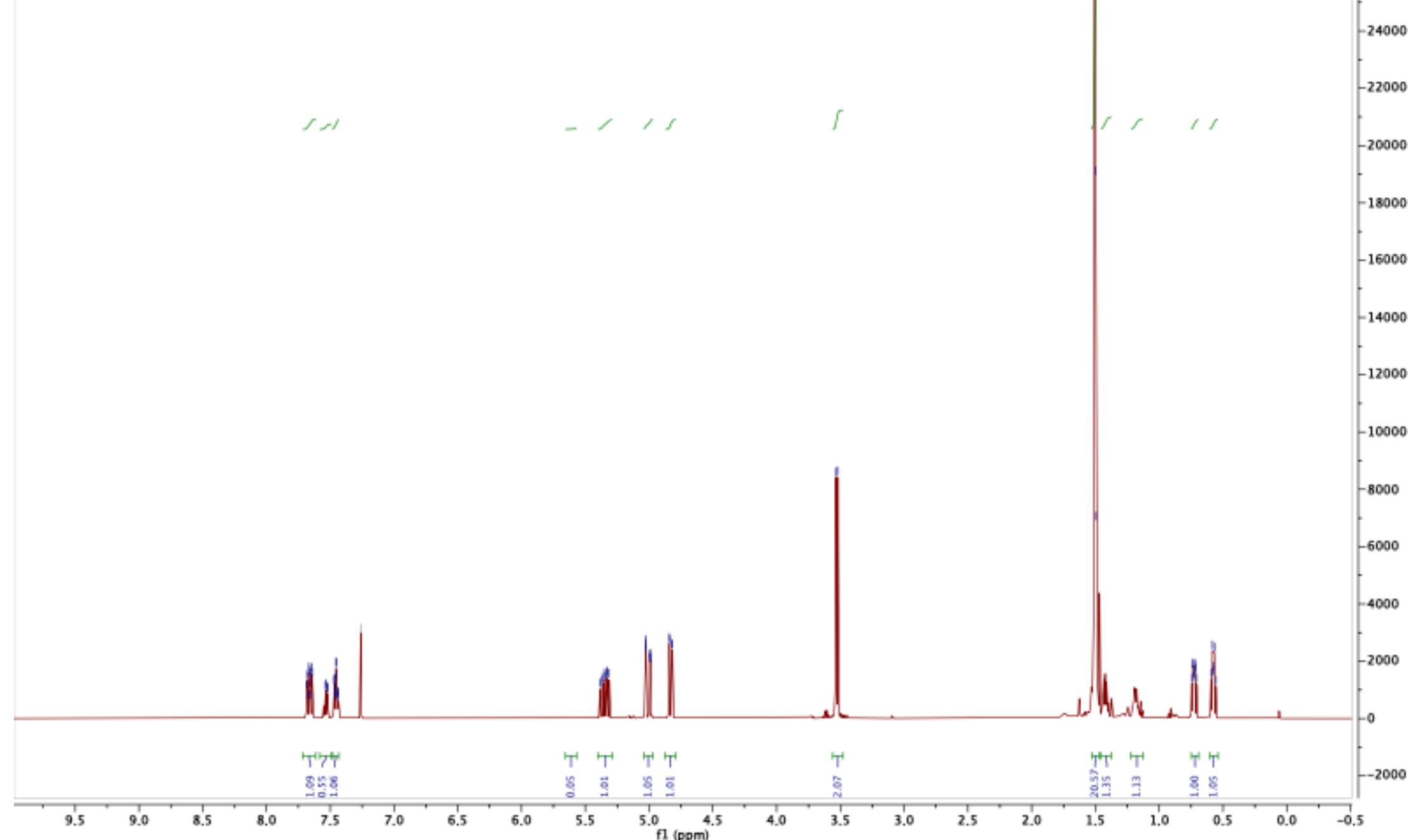


$(\mathrm{Boc})_{2} \mathrm{~N} \bigvee_{(-)-7}^{(\prime \prime \prime)}$

${ }^{13} \mathrm{C}\left\{{ }^{1} \mathrm{H}\right\} \mathrm{NMR}\left(126 \mathrm{MHz}, \mathrm{CDCl}_{3}\right)$

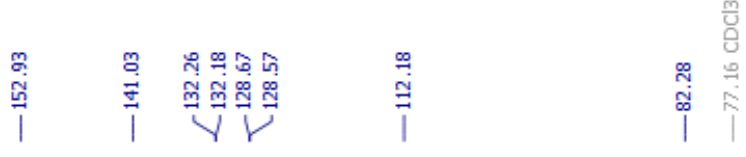

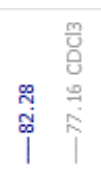

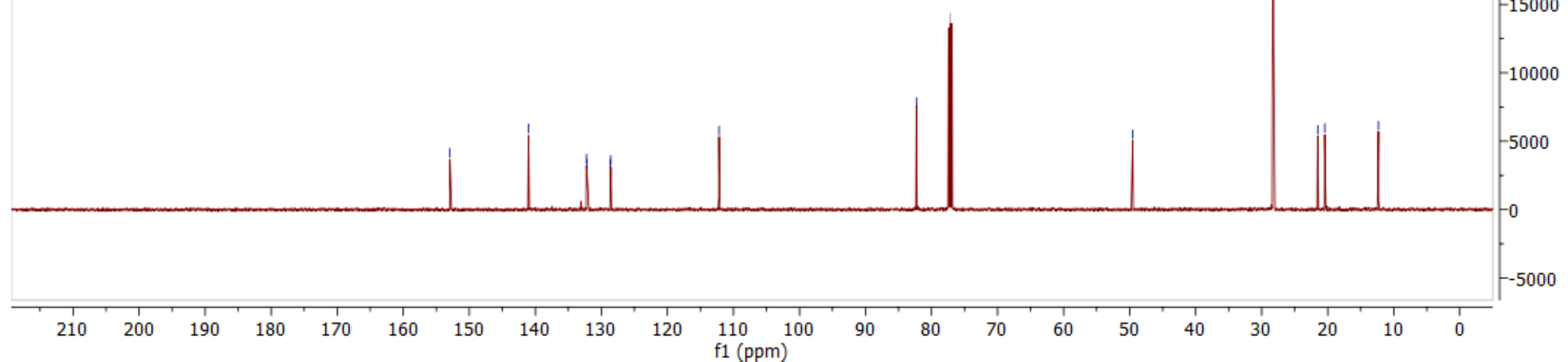


$(\mathrm{Boc})_{2} \mathrm{~N}^{\prime \prime \prime \prime}$

$(+)-7$

${ }^{1} \mathrm{H}$ NMR $\left(500 \mathrm{MHz}, \mathrm{CDCl}_{3}\right)$

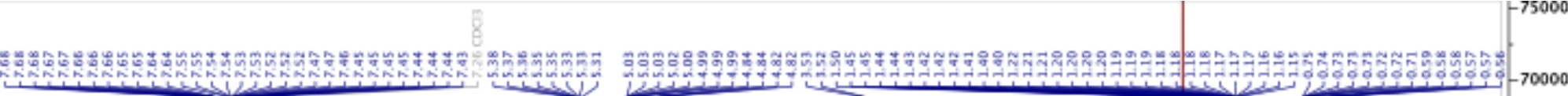

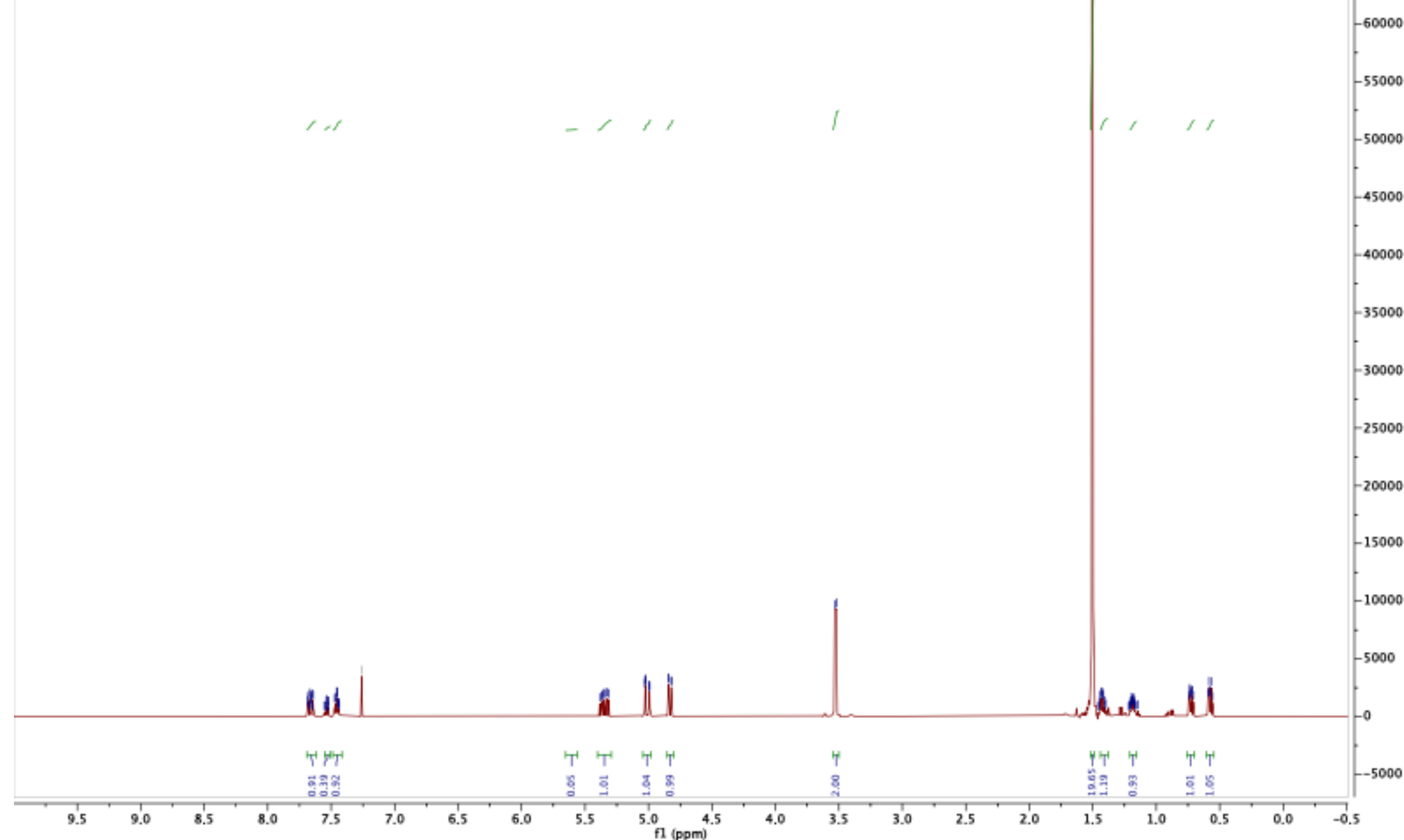

SI-27 
$(\mathrm{Boc})_{2} \mathrm{~N}^{\prime \prime \prime \prime \prime} \bigvee_{(+)-7}$

${ }^{13} \mathrm{C}\left\{{ }^{1} \mathrm{H}\right\}$ NMR (126 MHz, $\mathrm{CDCl}_{3}$ ) ll

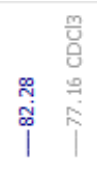

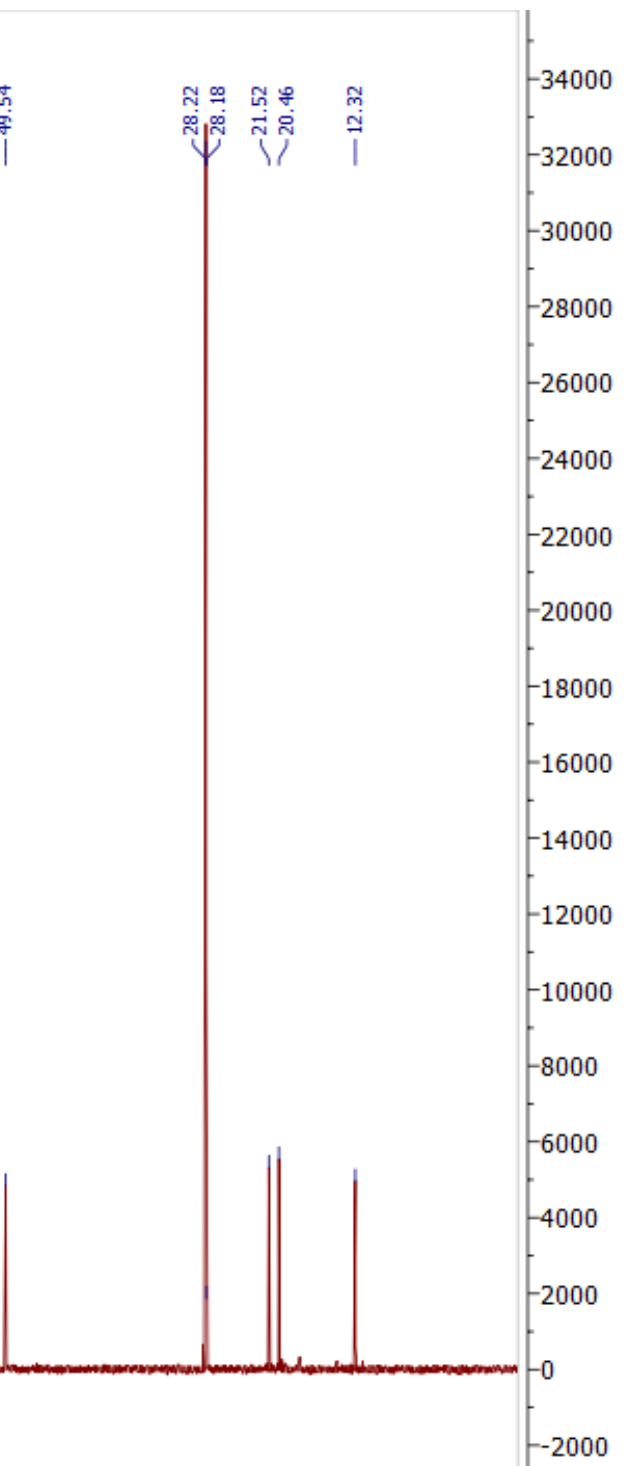

8000

000

4000

2000

0000

8000

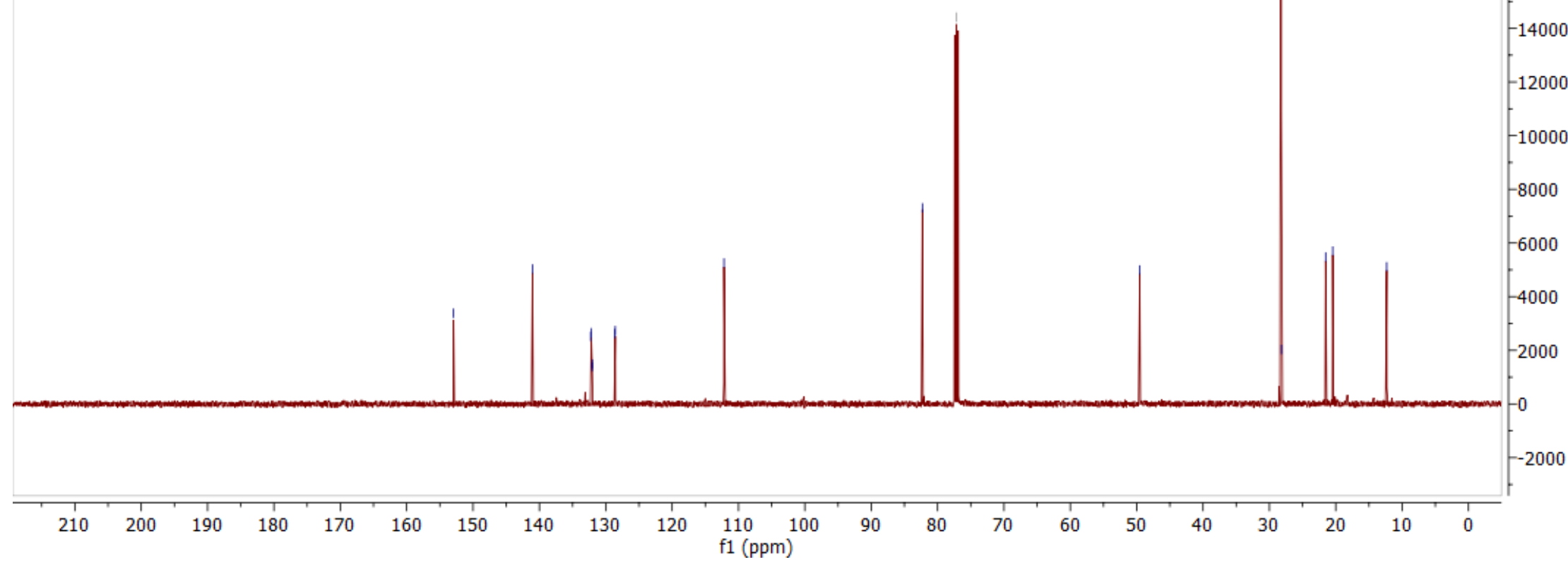

SI-28 


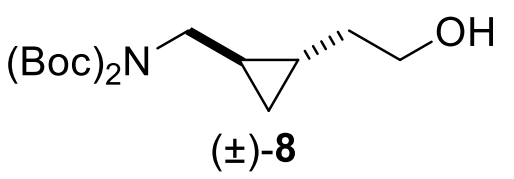

${ }^{1} \mathrm{H}$ NMR $\left(500 \mathrm{MHz}, \mathrm{CDCl}_{3}\right)$

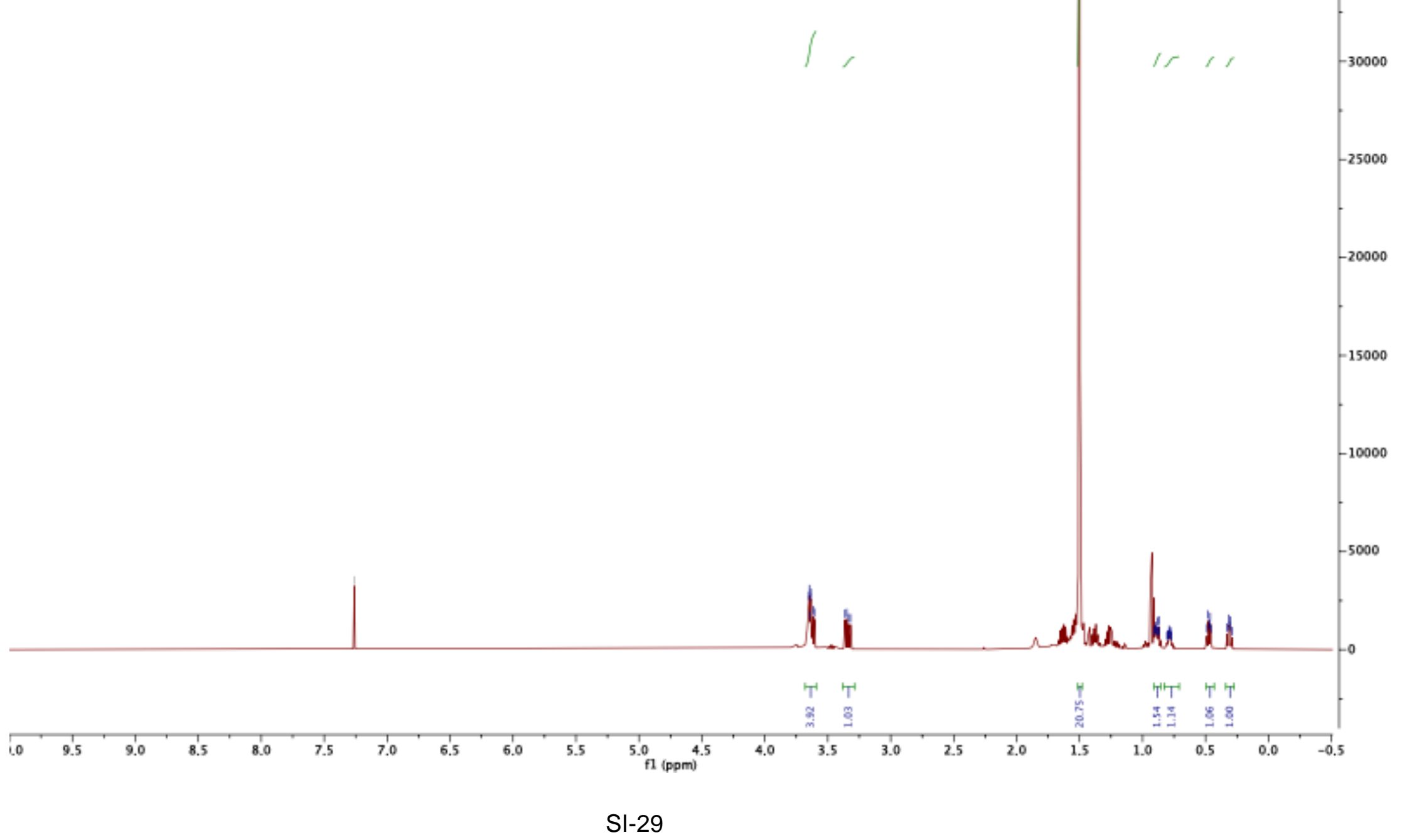




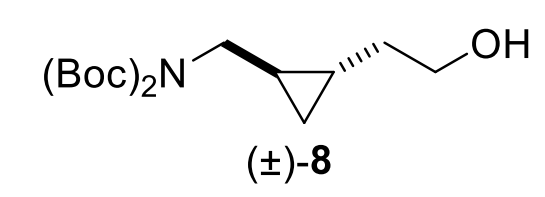

${ }^{13} \mathrm{C}\left\{{ }^{1} \mathrm{H}\right\} \operatorname{NMR}\left(126 \mathrm{MHz}, \mathrm{CDCl}_{3}\right)$

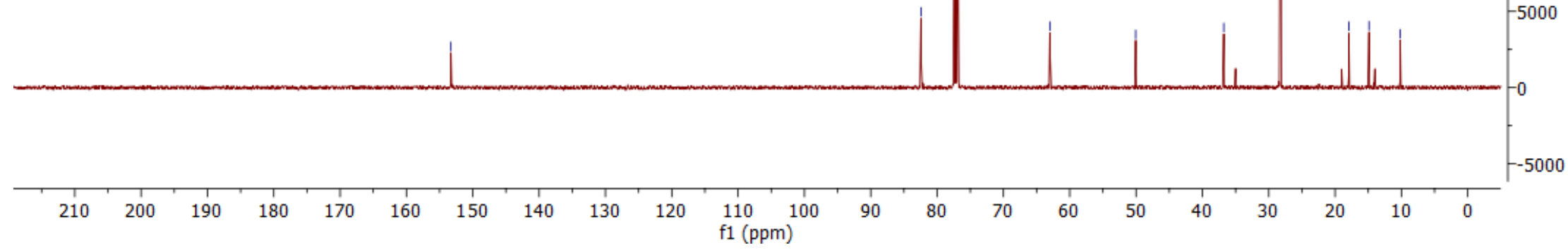



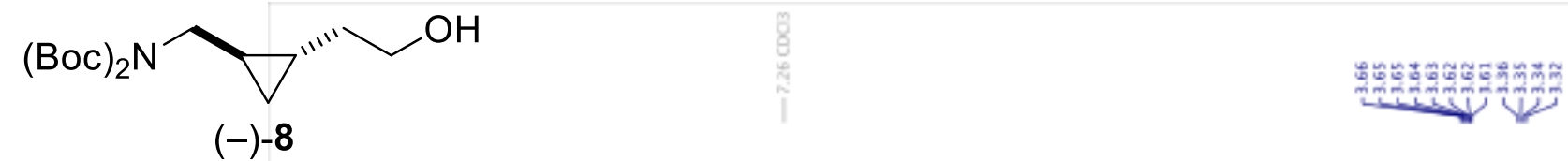

${ }^{1} \mathrm{H}$ NMR $\left(500 \mathrm{MHz}, \mathrm{CDCl}_{3}\right)$

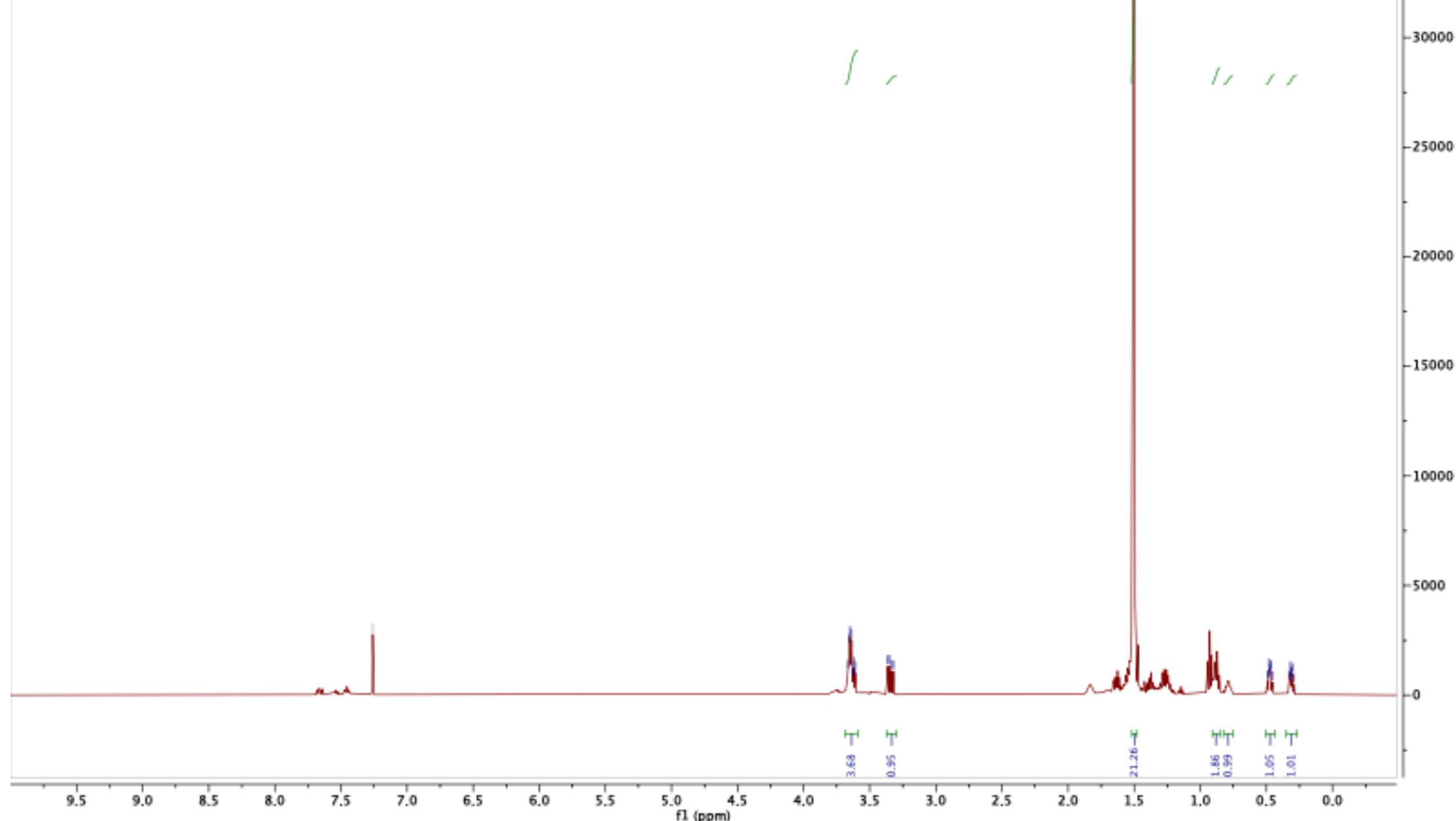

SI-31 


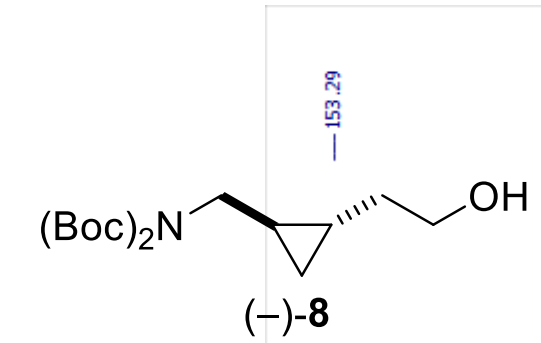

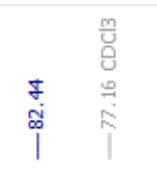
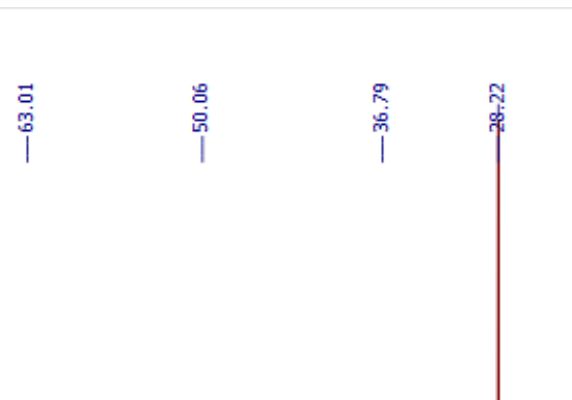

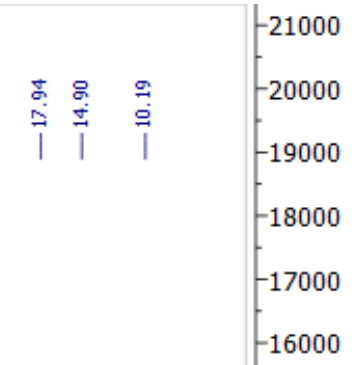

${ }^{13} \mathrm{C}\left\{{ }^{1} \mathrm{H}\right\}$ NMR $\left(126 \mathrm{MHz}, \mathrm{CDCl}_{3}\right)$

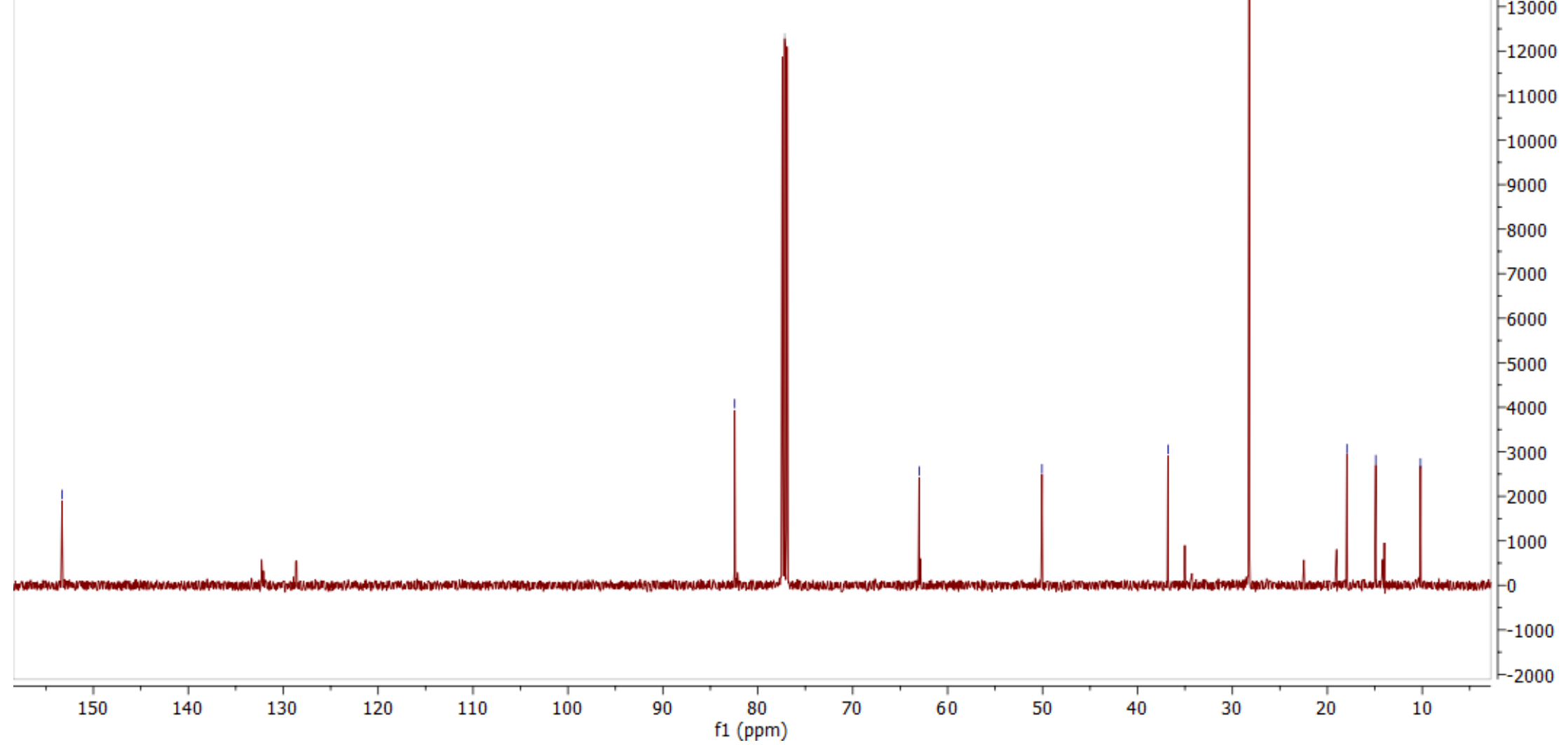

SI-32 

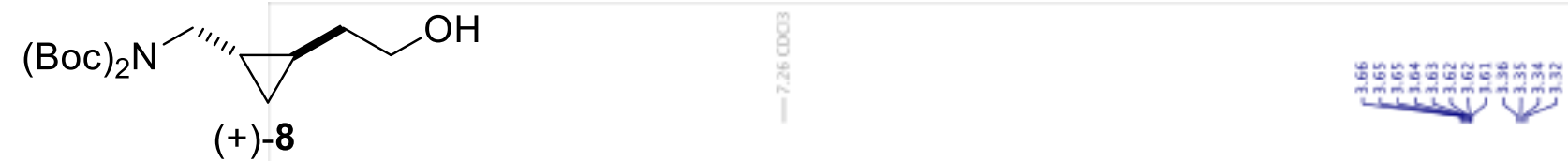

${ }^{1} \mathrm{H}$ NMR $\left(500 \mathrm{MHz}, \mathrm{CDCl}_{3}\right)$

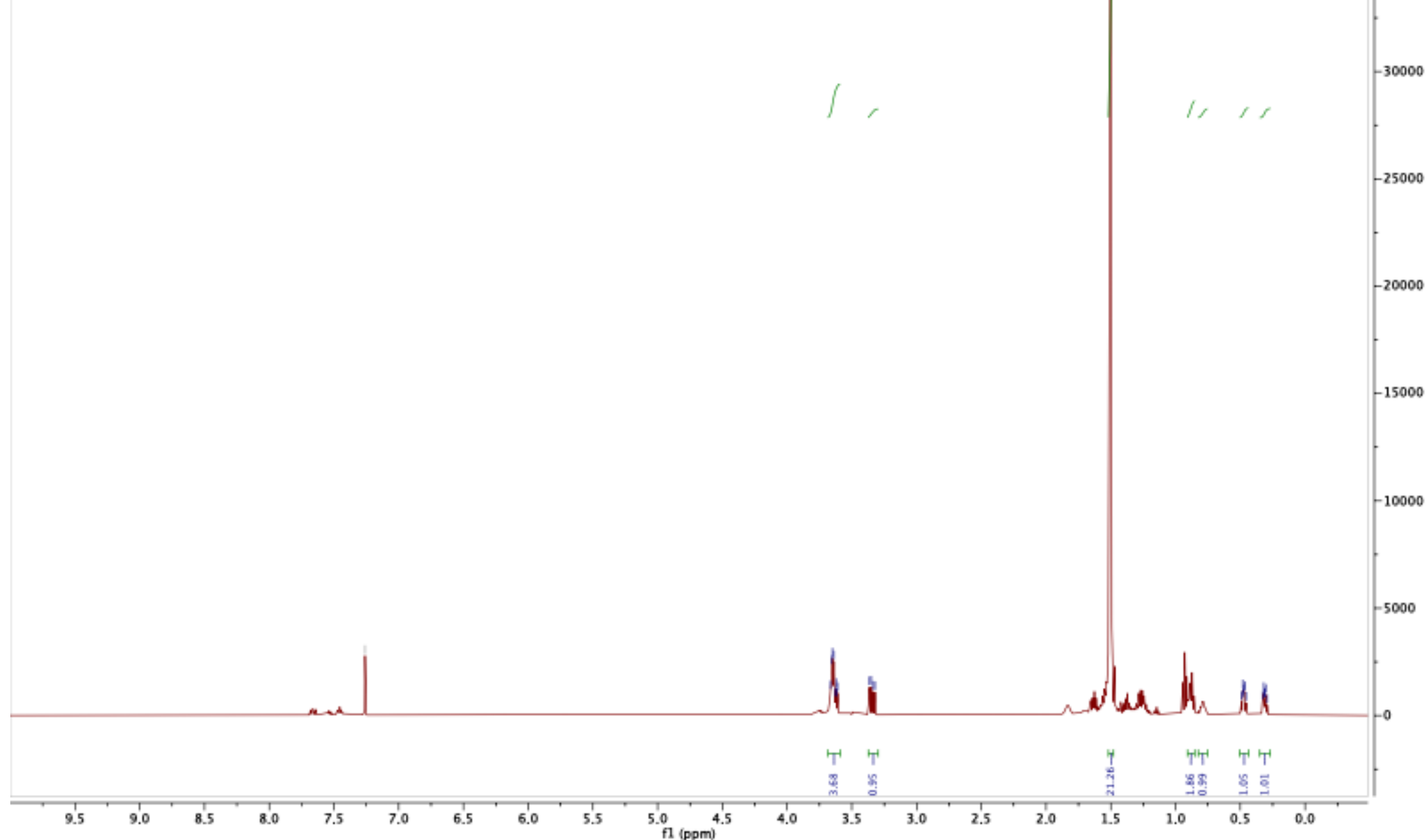




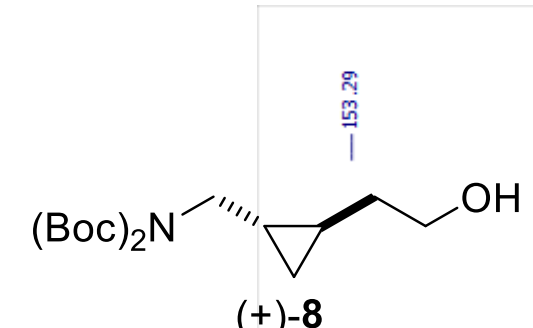

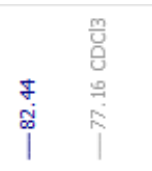
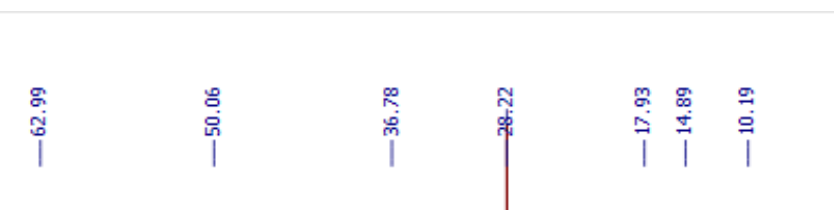

$-24000$

$(+)$

${ }^{13} \mathrm{C}\left\{{ }^{1} \mathrm{H}\right\}$ NMR $\left(126 \mathrm{MHz}, \mathrm{CDCl}_{3}\right)$

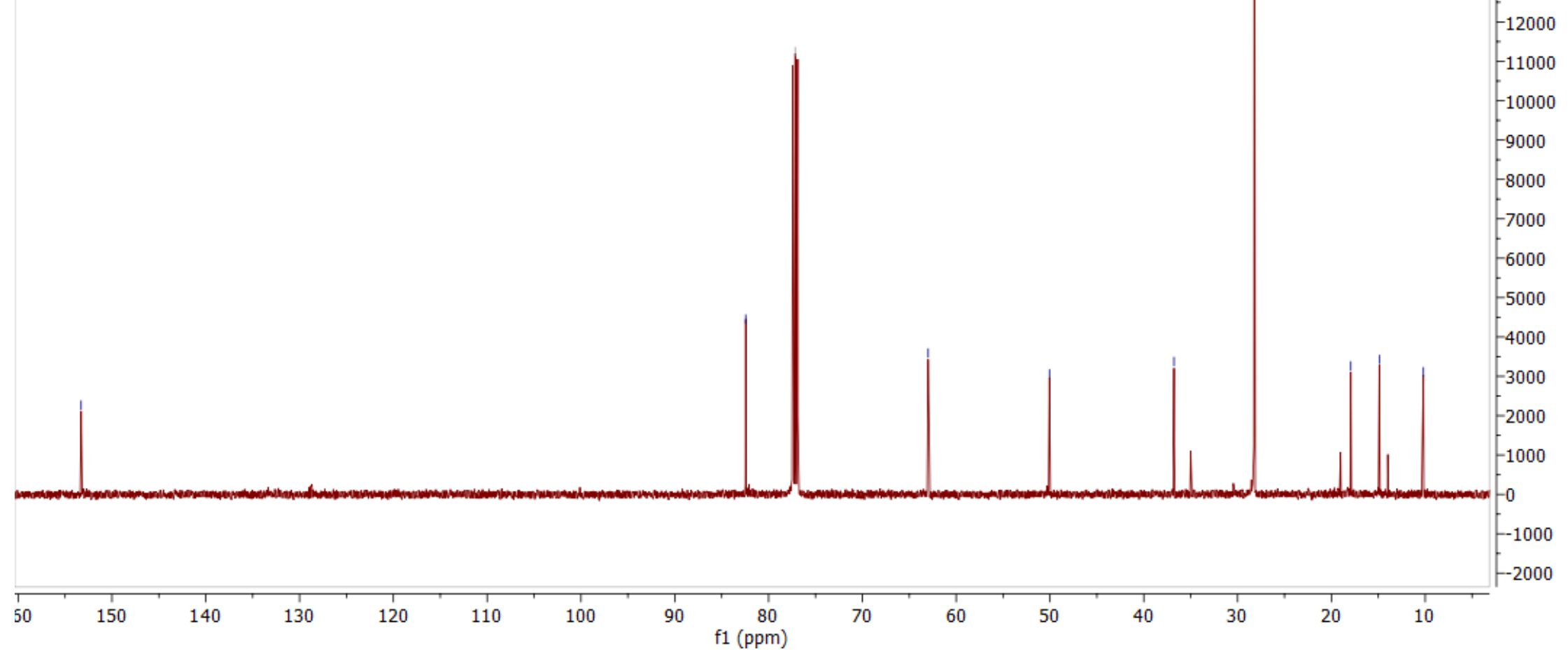

SI-34 


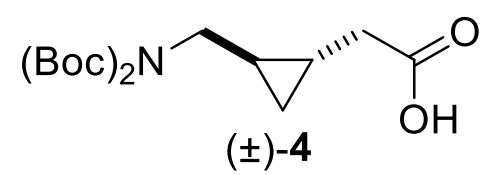

${ }^{1} \mathrm{H}$ NMR $\left(500 \mathrm{MHz}, \mathrm{CDCl}_{3}\right)$

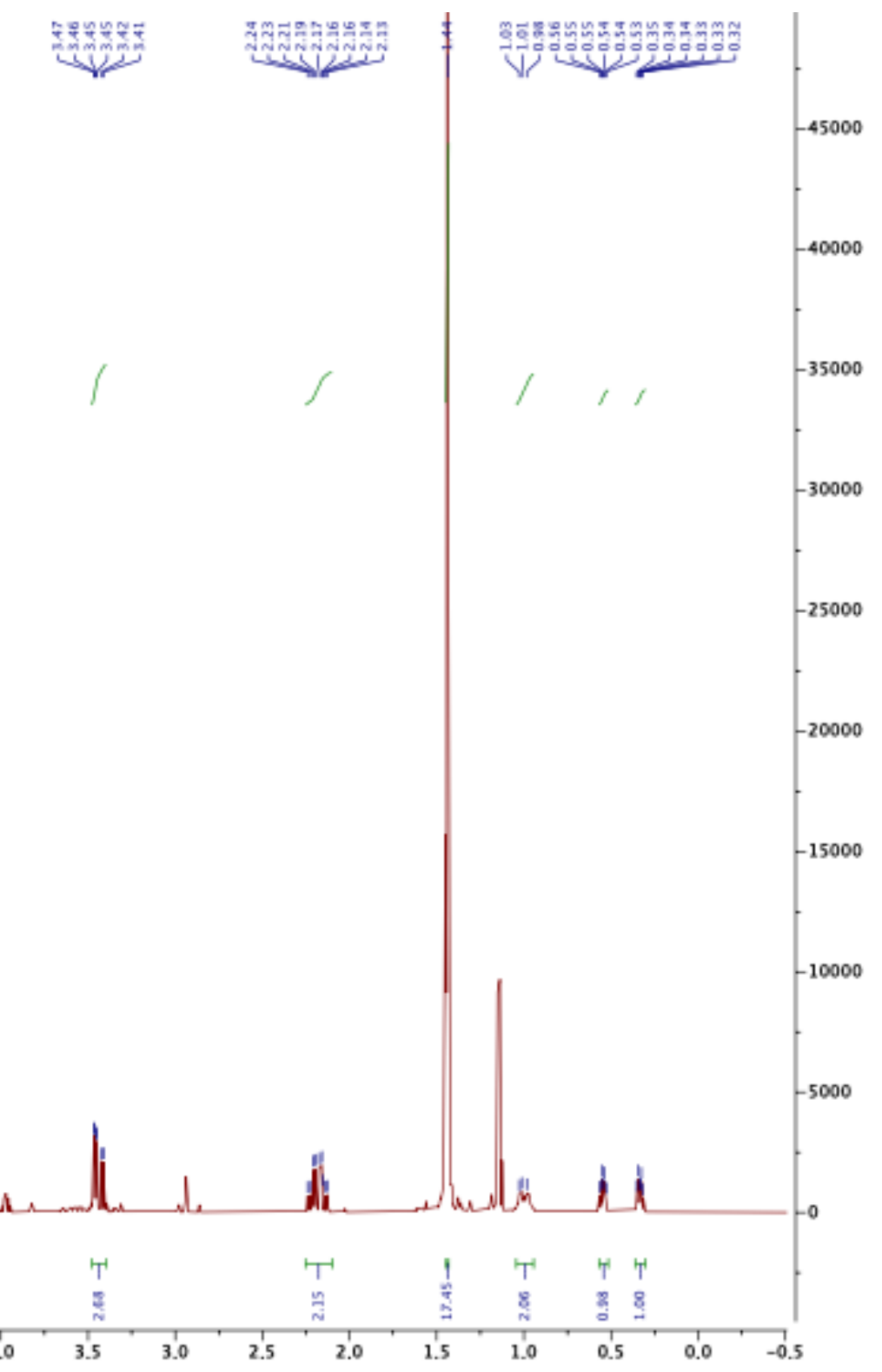

SI-35 


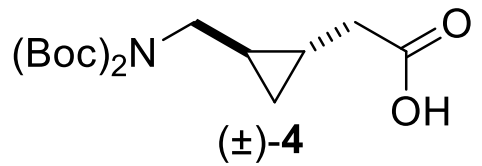

${ }^{13} \mathrm{C}\left\{{ }^{1} \mathrm{H}\right\}$ NMR $\left(126 \mathrm{MHz}, \mathrm{CDCl}_{3}\right)$
550 


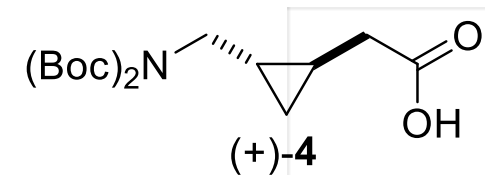

${ }^{1} \mathrm{H}$ NMR $\left(500 \mathrm{MHz}, \mathrm{CDCl}_{3}\right)$

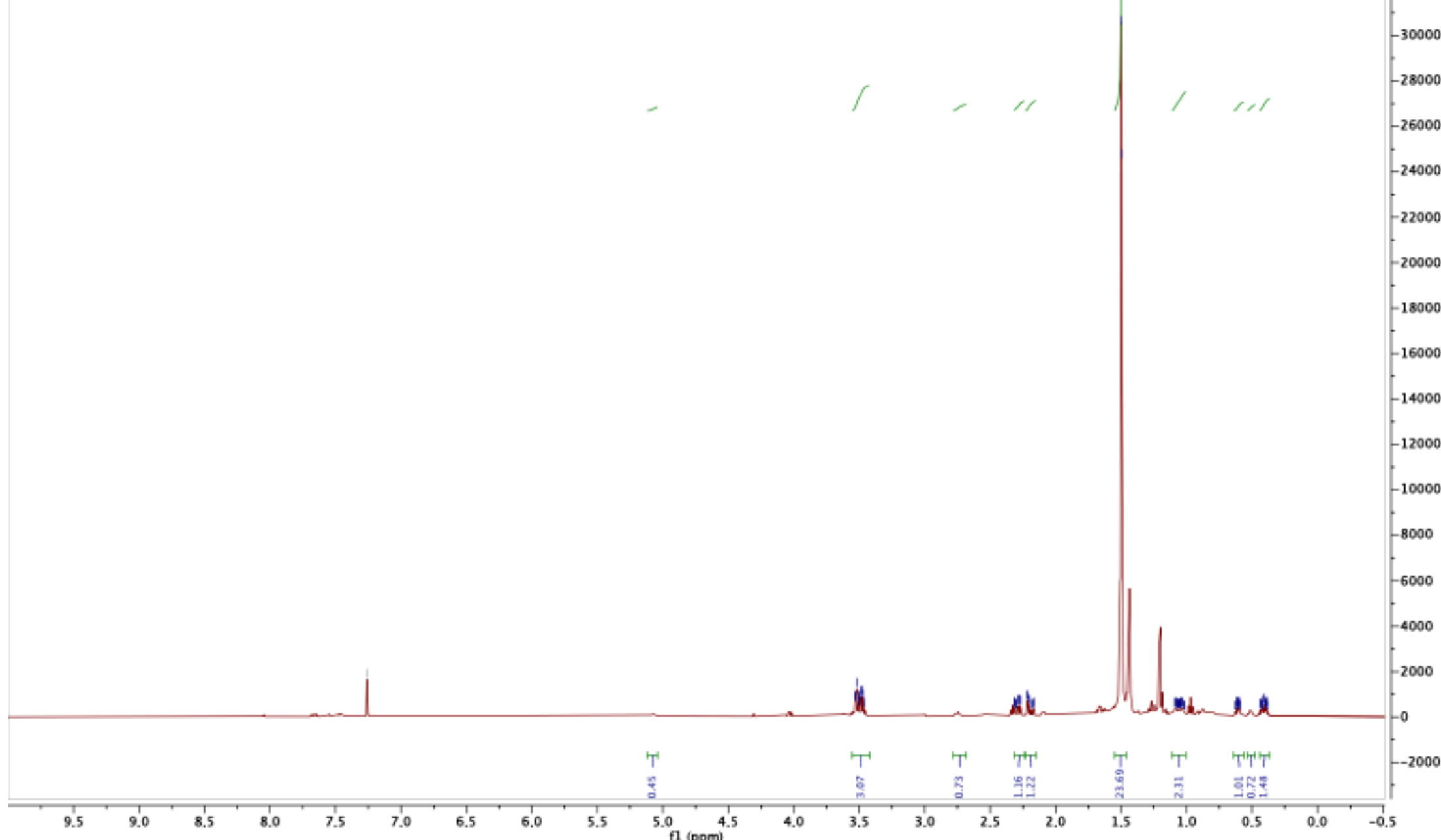




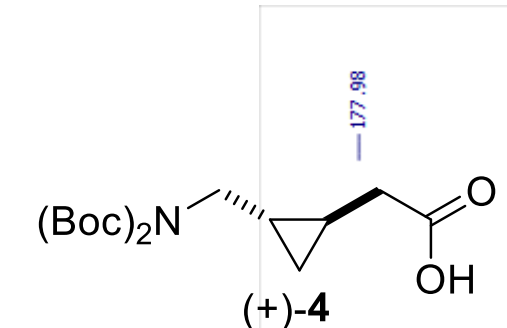

${ }^{13} \mathrm{C}\left\{{ }^{1} \mathrm{H}\right\}$ NMR $\left(126 \mathrm{Mz}, \mathrm{CDCl}_{3}\right)$

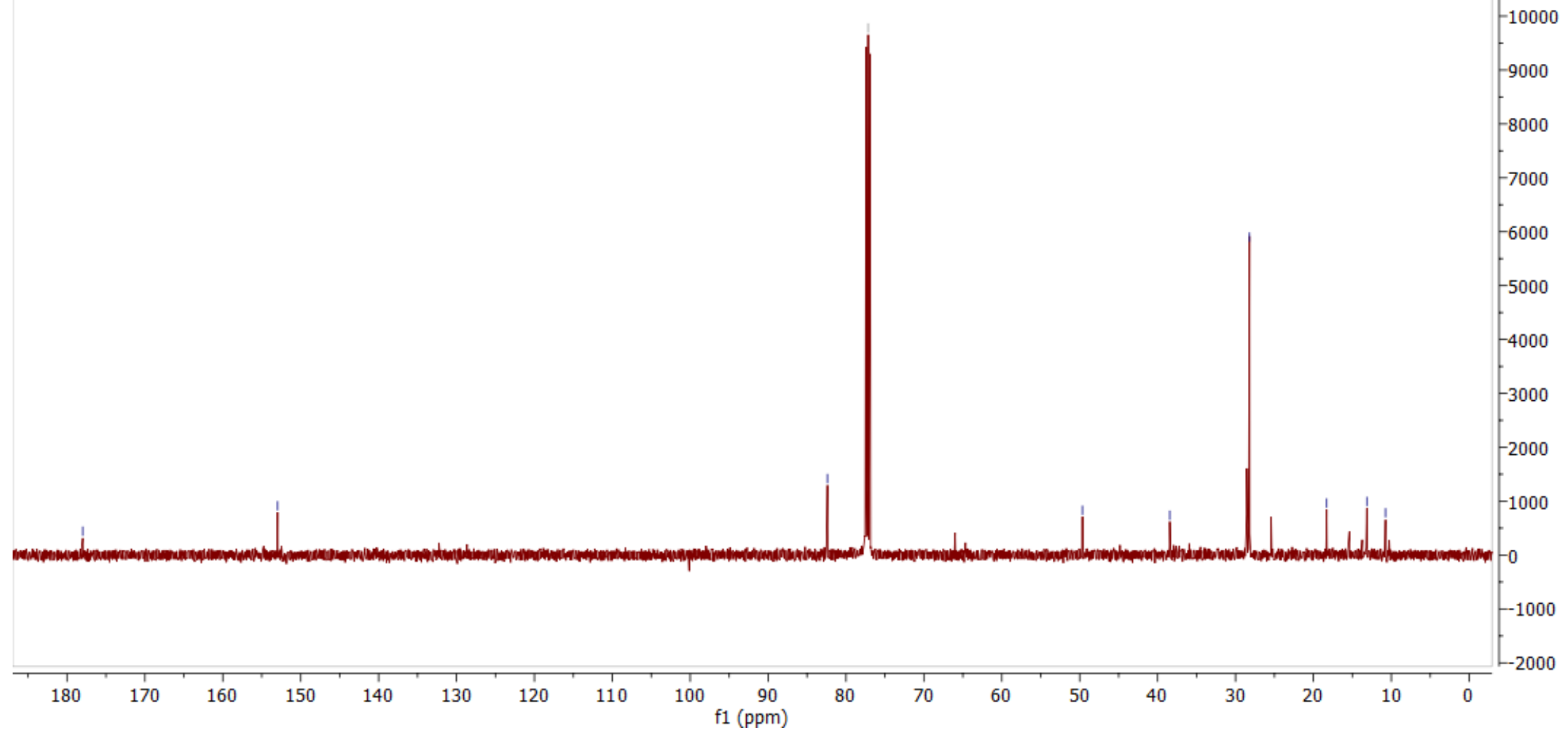




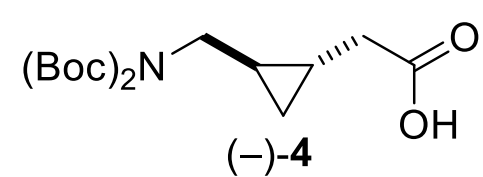

${ }^{1} \mathrm{H}$ NMR $\left(500 \mathrm{MHz}, \mathrm{CDCl}_{3}\right)$

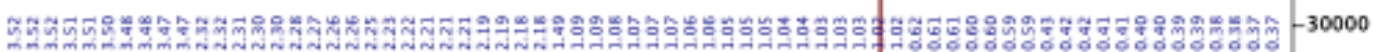
r

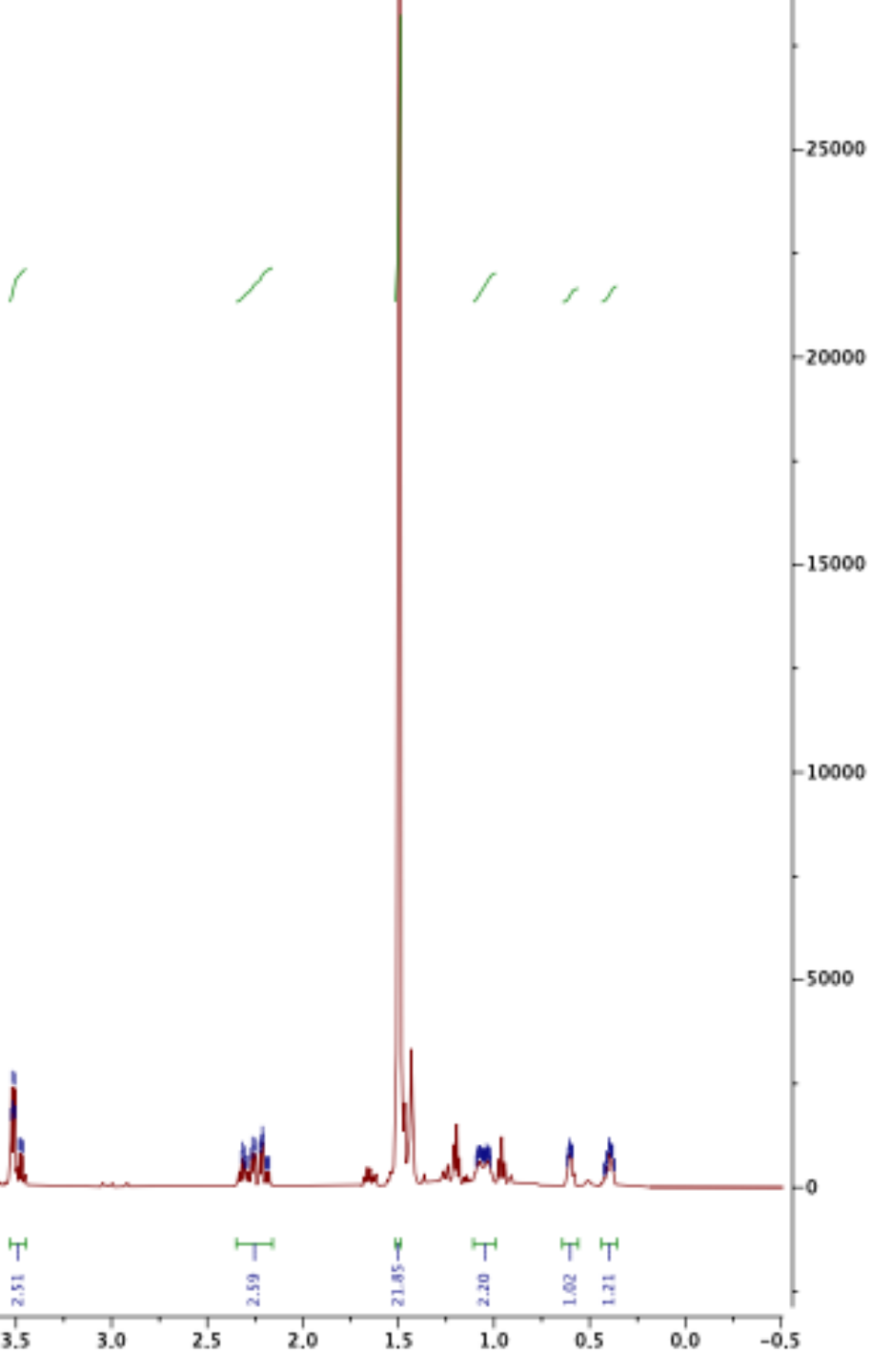

SI-39 


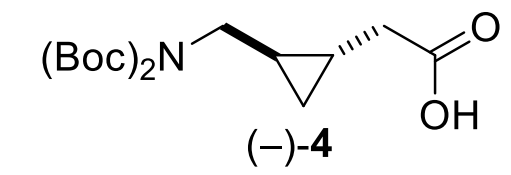

${ }^{13} \mathrm{C}\left\{{ }^{1} \mathrm{H}\right\} \operatorname{NMR}\left(126 \mathrm{MHz}, \mathrm{CDCl}_{3}\right)$
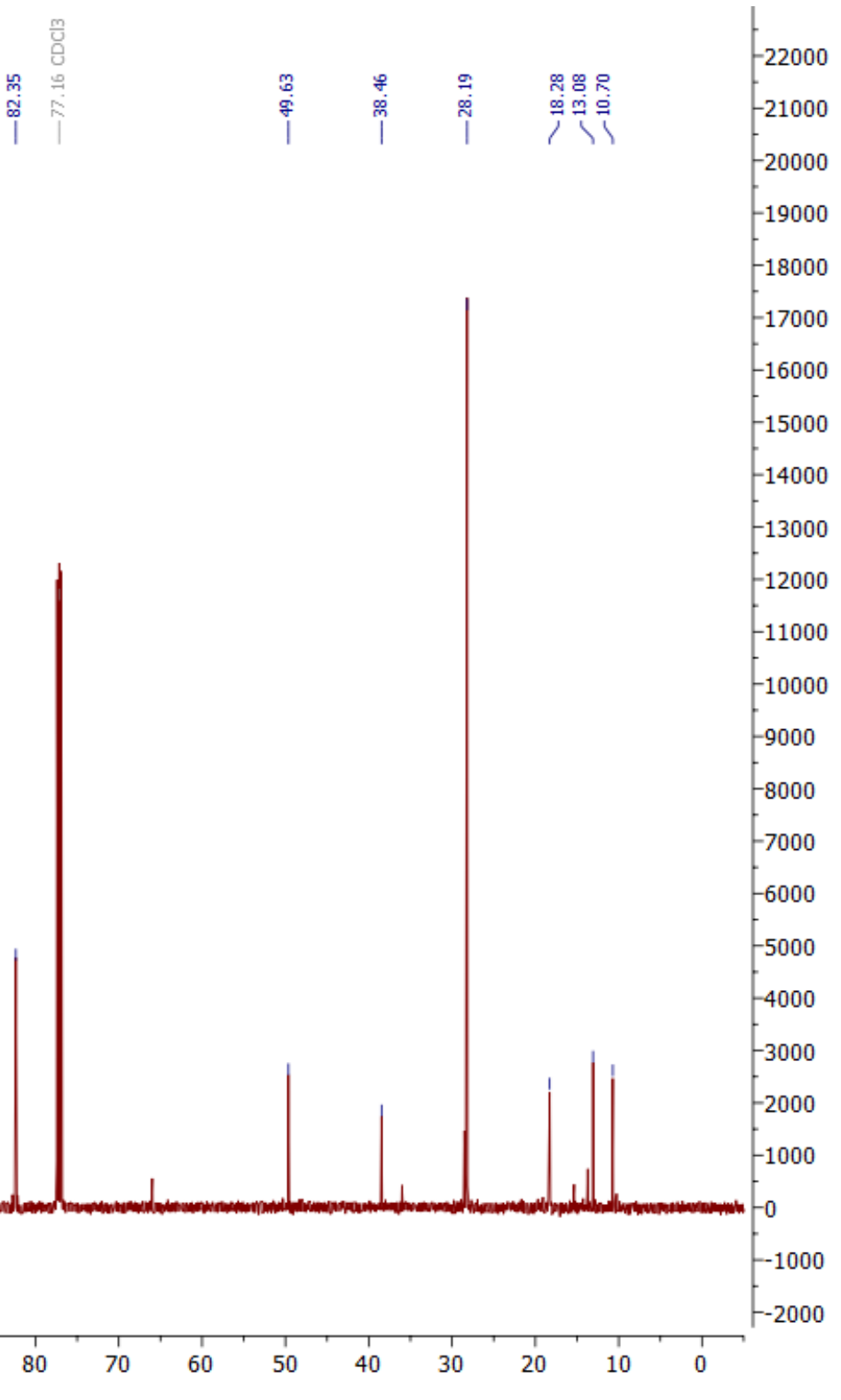

SI-40 

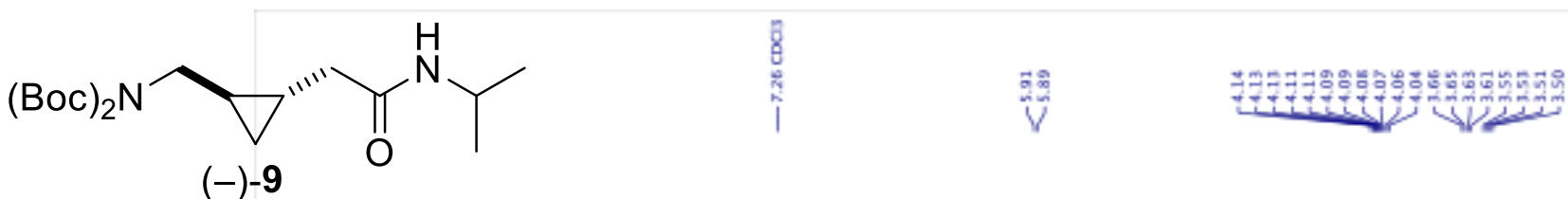

${ }^{1} \mathrm{H}$ NMR $\left(400 \mathrm{MHz}, \mathrm{CDCl}_{3}\right)$

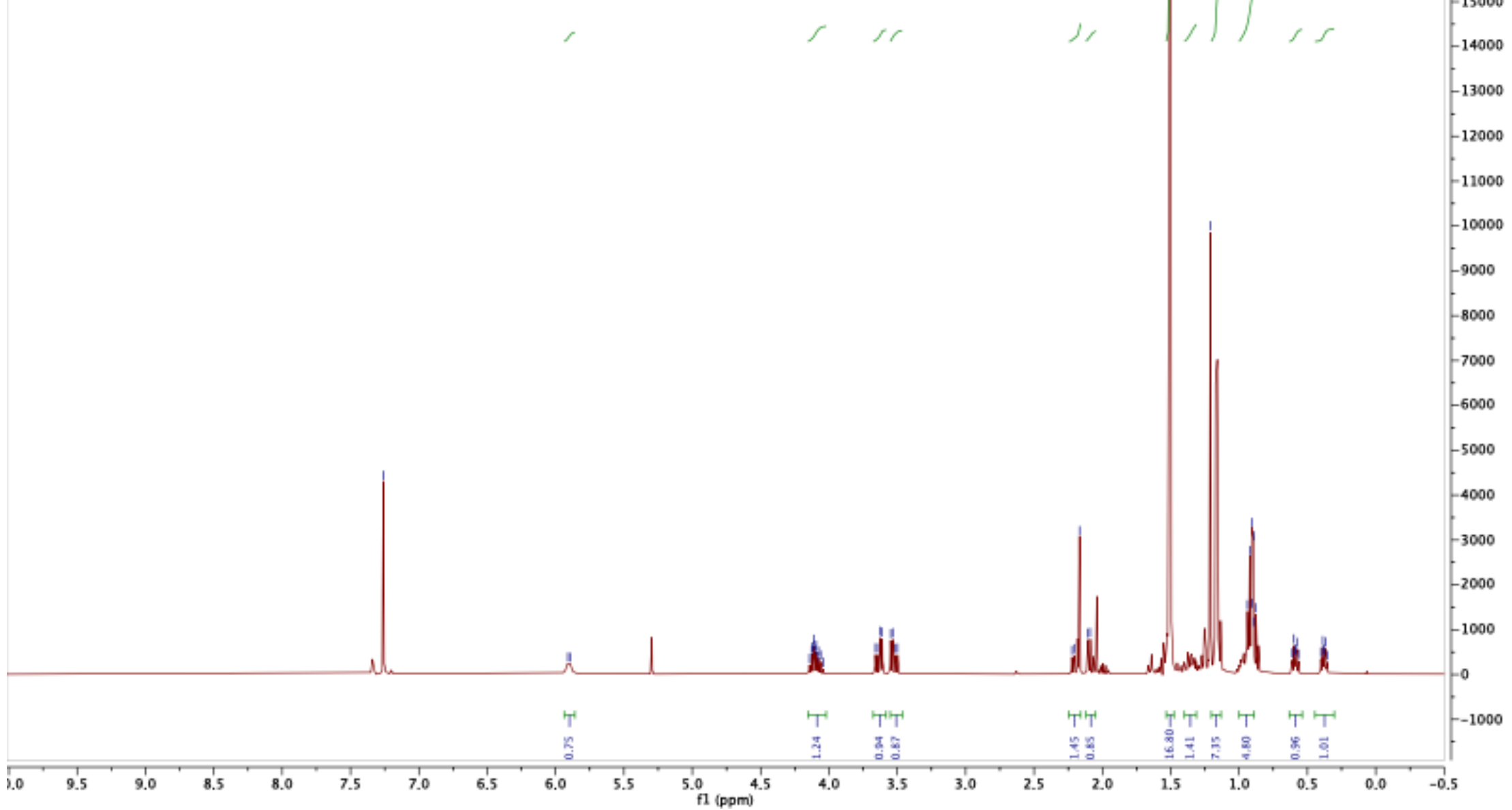




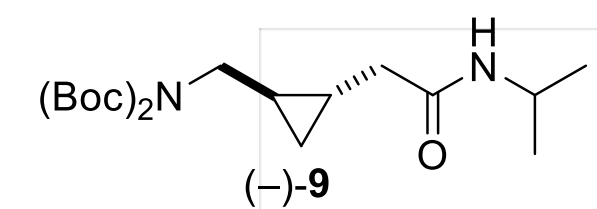

$\stackrel{\sqrt{1}}{\sqrt[1]{2}}$

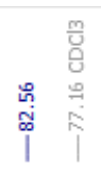

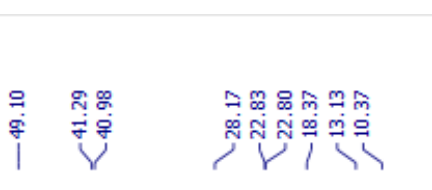

$-230000$

$-220000$

210000

$-200000$

${ }^{13} \mathrm{C}\left\{{ }^{1} \mathrm{H}\right\}$ NMR $\left(101 \mathrm{MHz}, \mathrm{CDCl}_{3}\right)$

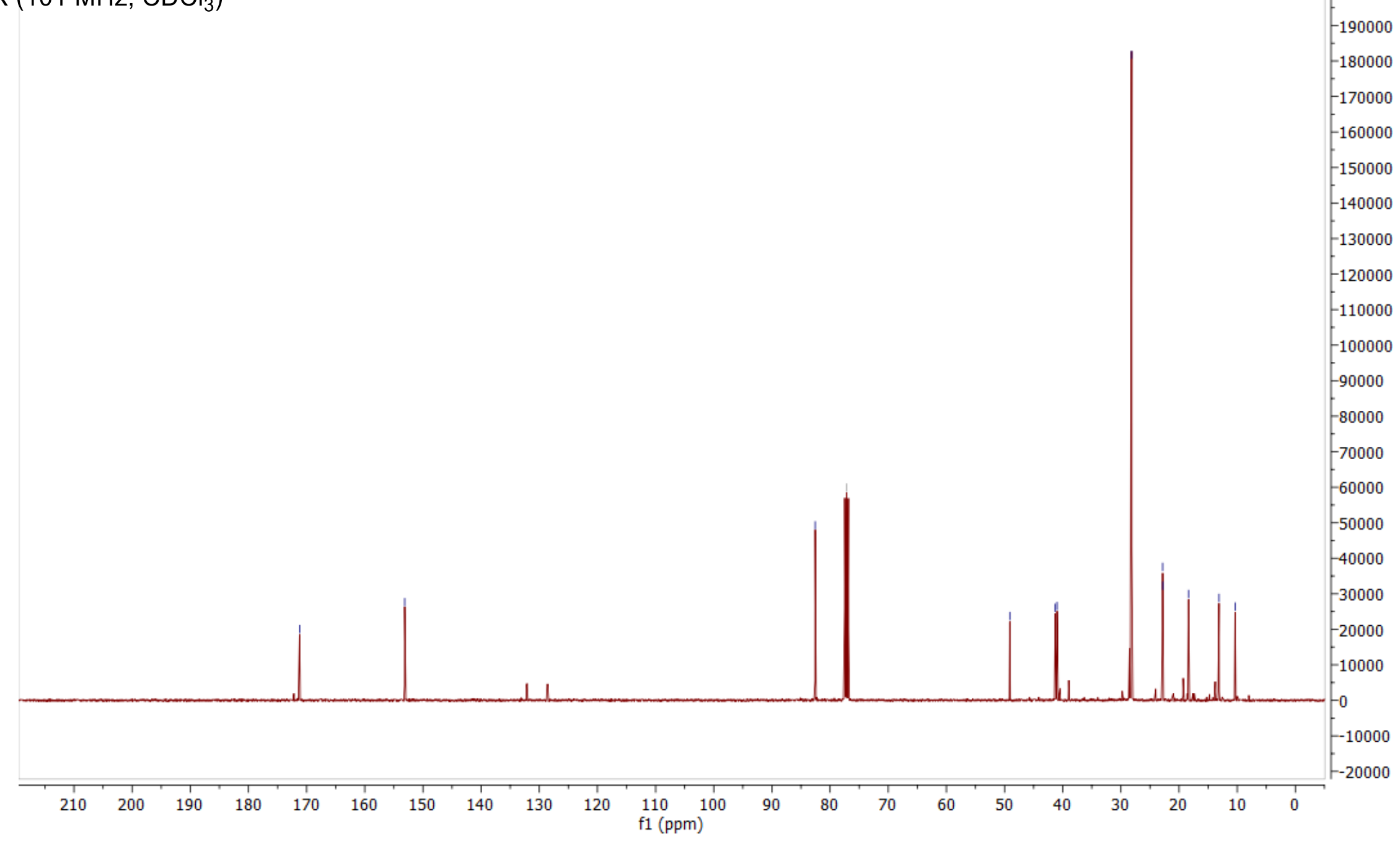




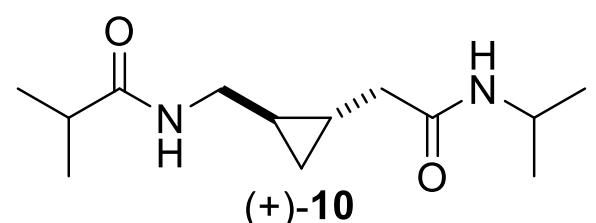

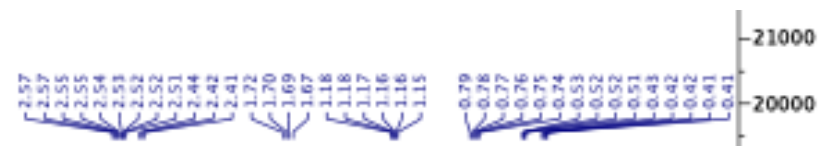

$$
\text { (+)-10 }
$$

${ }^{1} \mathrm{H}$ NMR $\left(500 \mathrm{MHz}, \mathrm{CDCl}_{3}\right)$

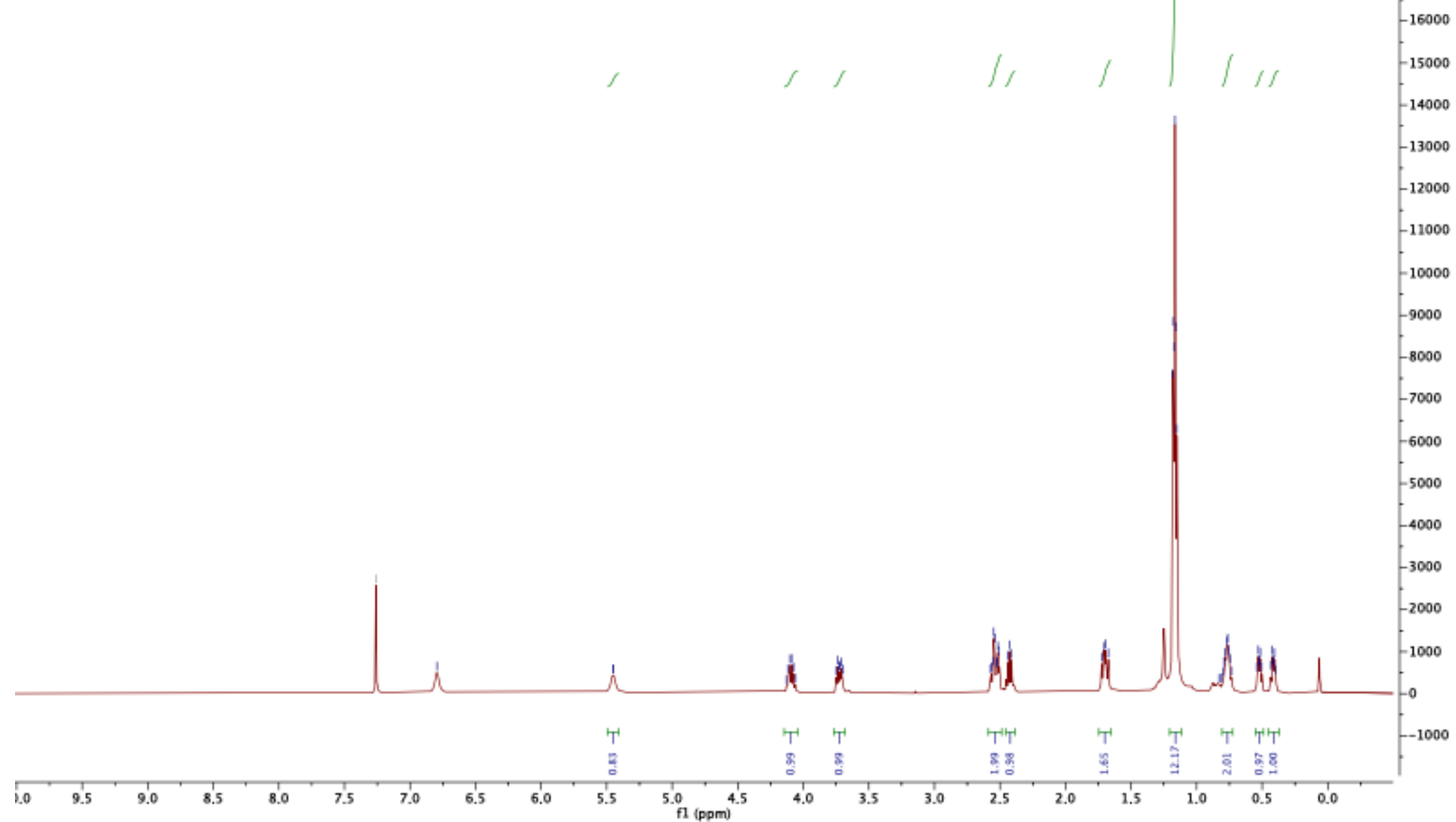




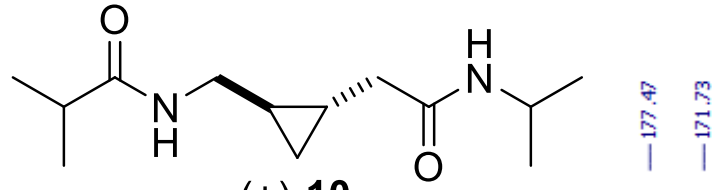

(+)-10

${ }^{13} \mathrm{C}\left\{{ }^{1} \mathrm{H}\right\}$ NMR $\left(126 \mathrm{MHz}, \mathrm{CDCl}_{3}\right)$

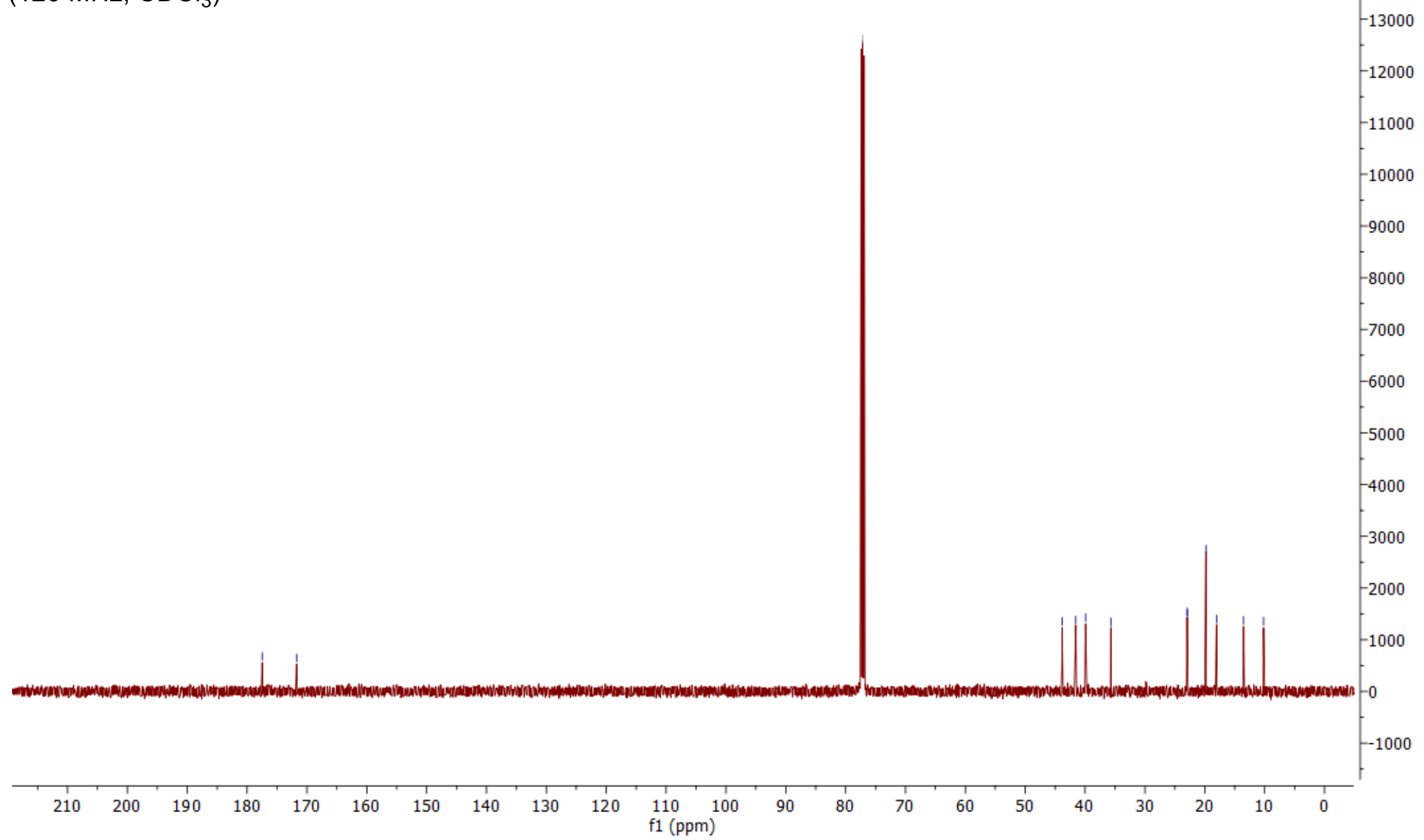


$(\text { Boc })_{2} \mathrm{~N} \searrow_{(-)-11}^{\prime \prime \prime \prime} \prod_{O}^{N}$

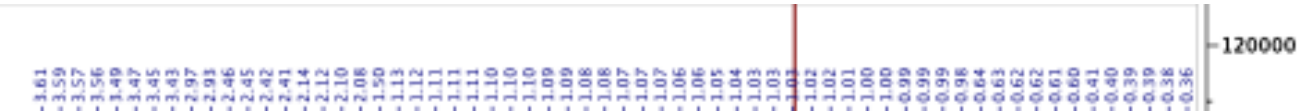

(-)-11

${ }^{1} \mathrm{H}$ NMR $\left(400 \mathrm{MHz}, \mathrm{CDCl}_{3}\right)$

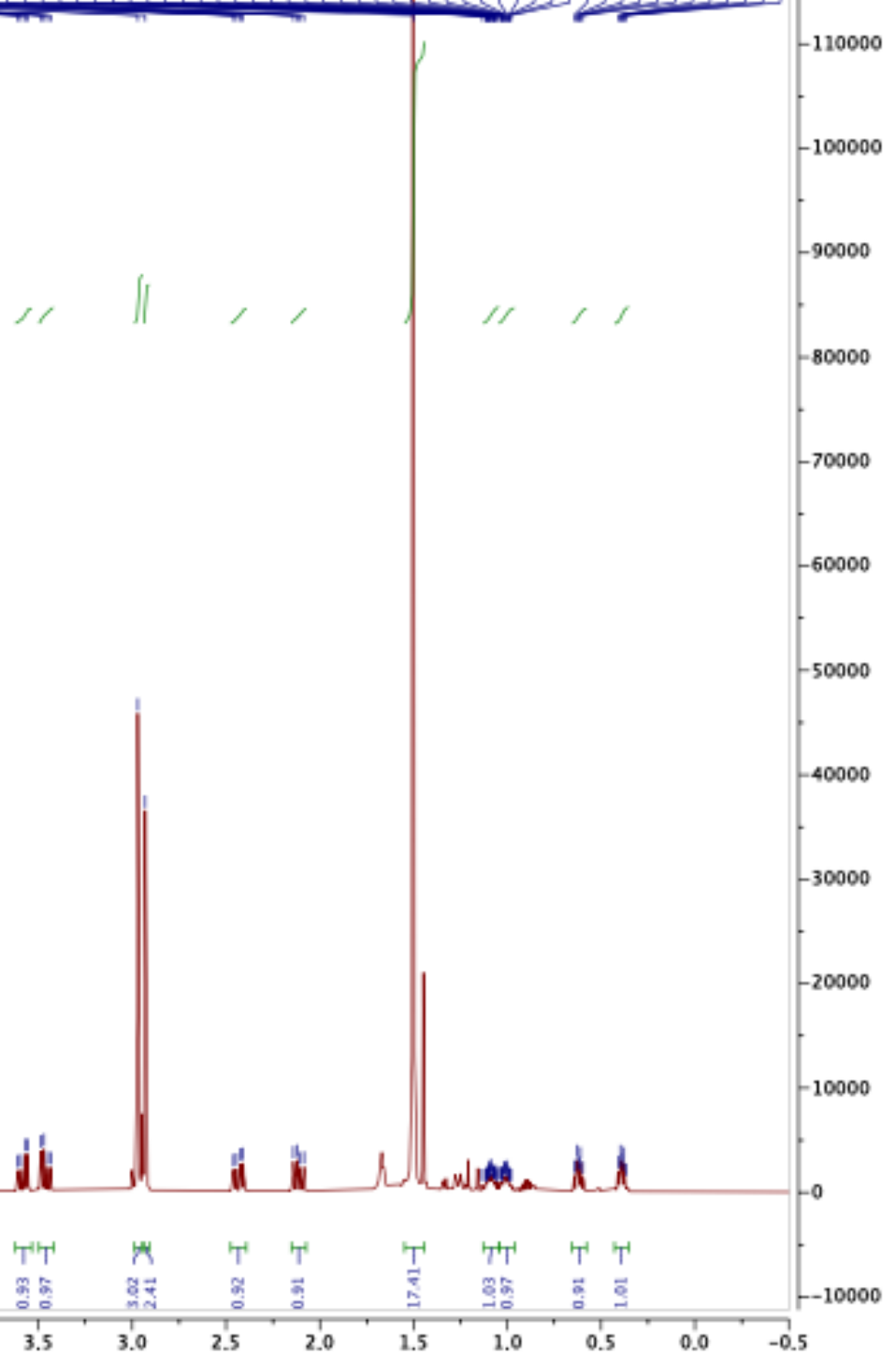

SI-45 


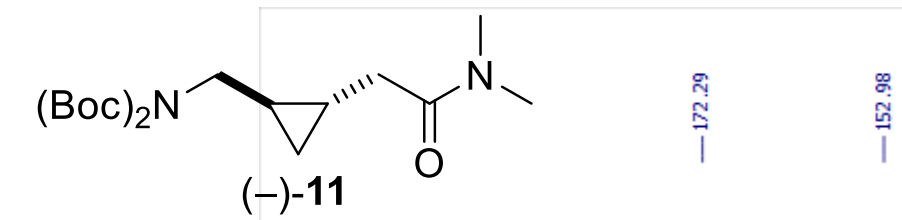

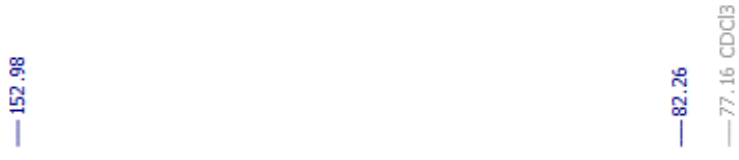

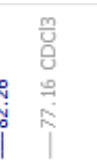

$(-)-11$

${ }^{13} \mathrm{C}\left\{{ }^{1} \mathrm{H}\right\}$ NMR $\left(101 \mathrm{Mz}, \mathrm{CDCl}_{3}\right)$

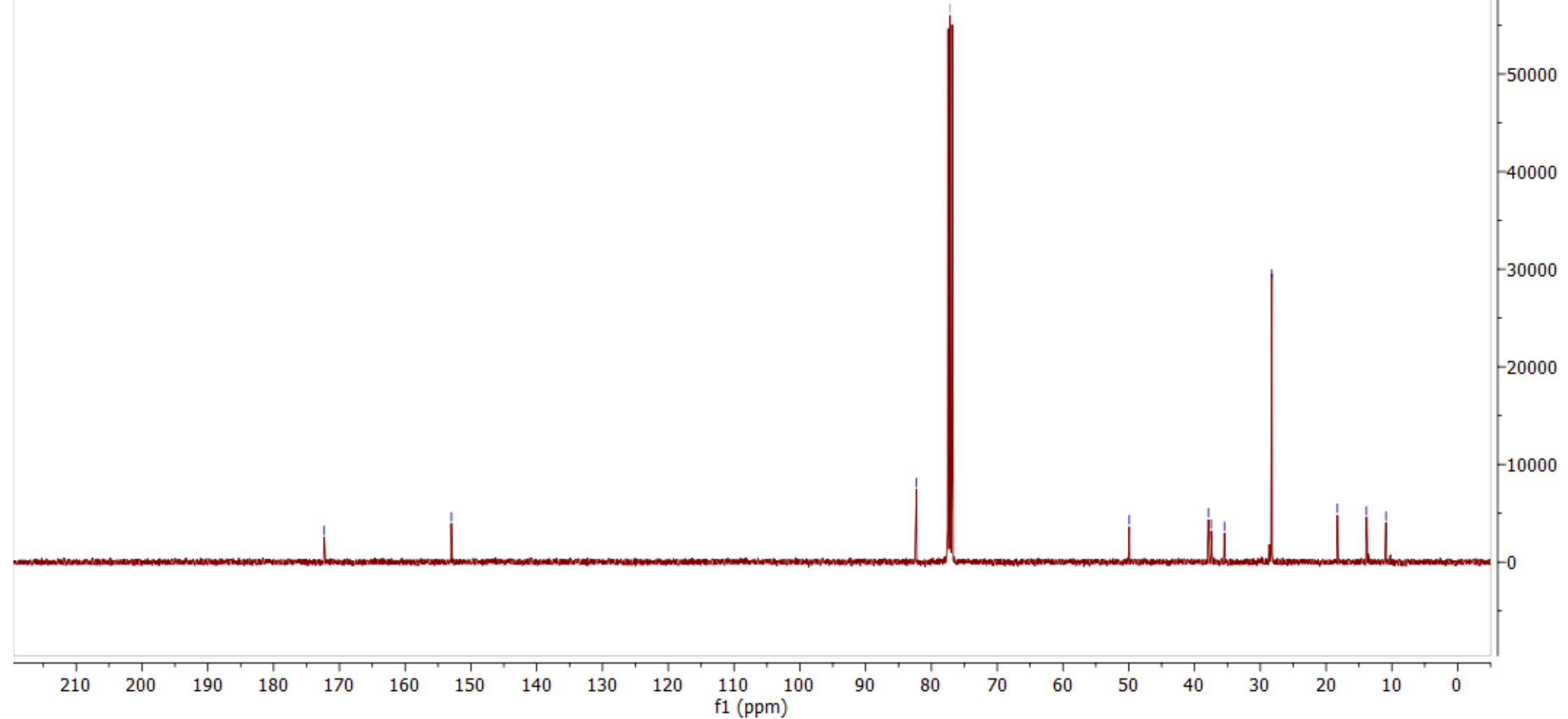




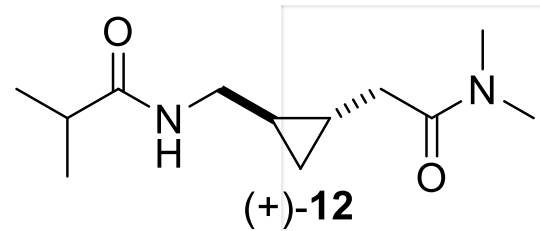

$$
(+)-12
$$

${ }^{1} \mathrm{H}$ NMR $\left(500 \mathrm{MHz}, \mathrm{CDCl}_{3}\right)$

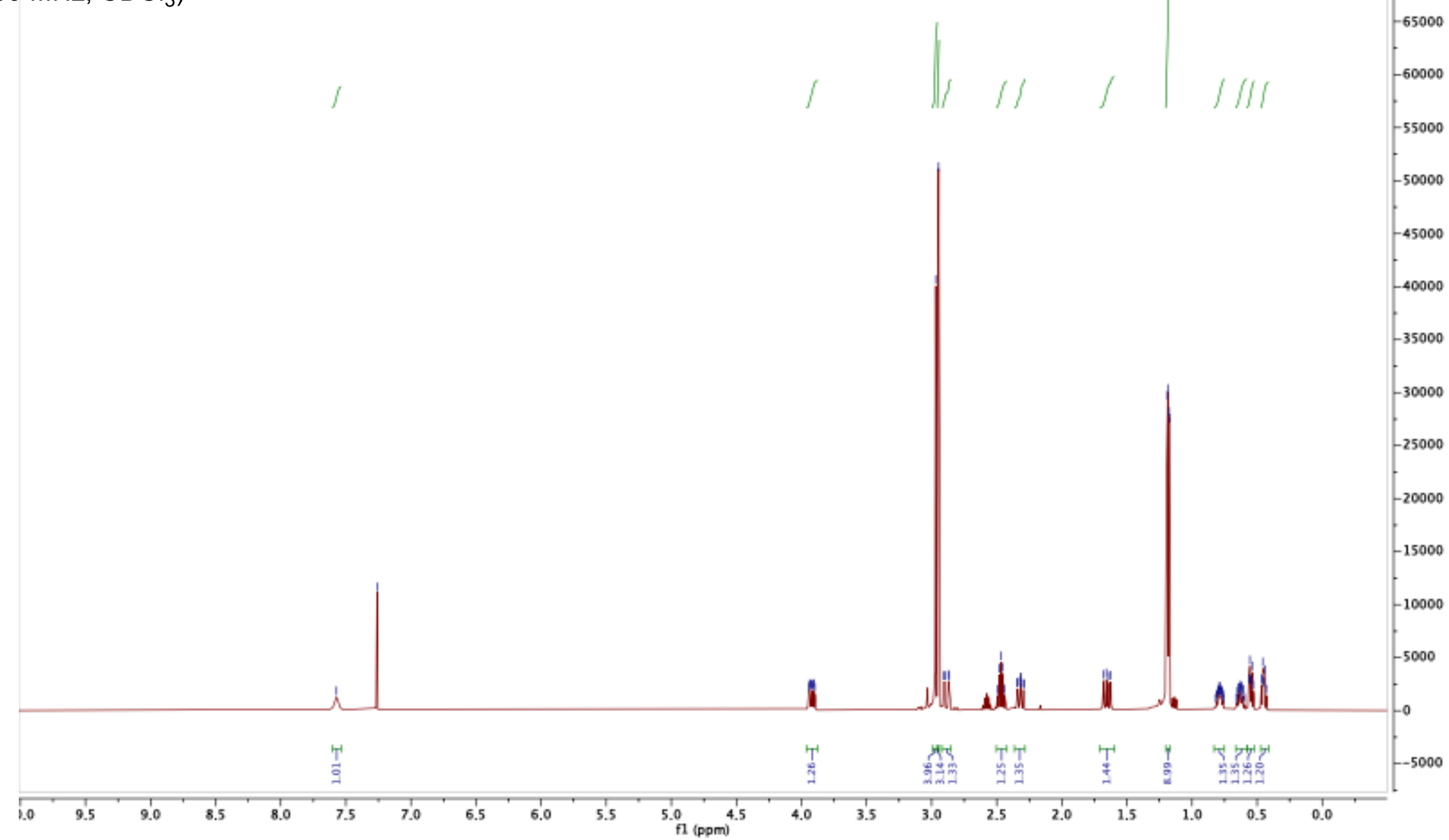

SI-47 


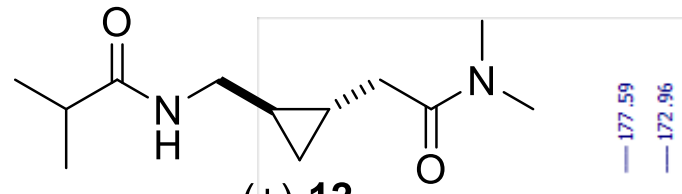

(+)-12

${ }^{13} \mathrm{C}\left\{{ }^{1} \mathrm{H}\right\}$ NMR $\left(126 \mathrm{MHz}, \mathrm{CDCl}_{3}\right)$

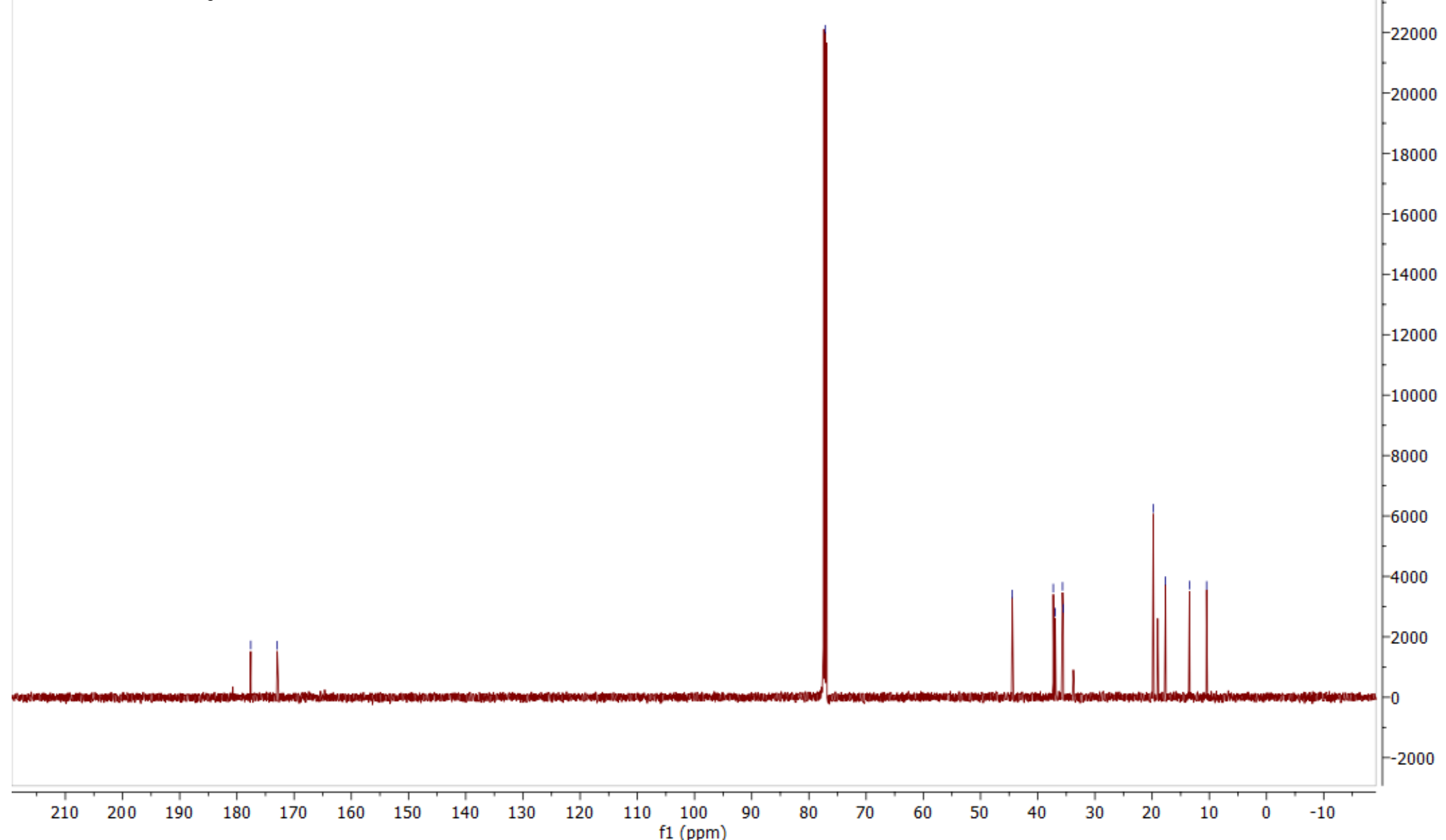




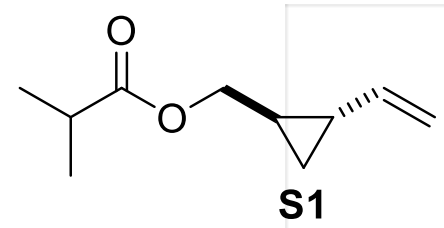

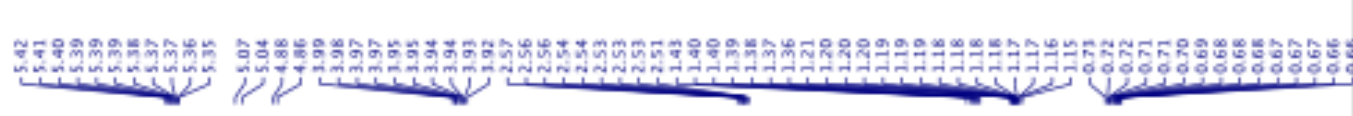

$-70000$

${ }^{1} \mathrm{H}$ NMR $\left(500 \mathrm{MHz}, \mathrm{CDCl}_{3}\right)$

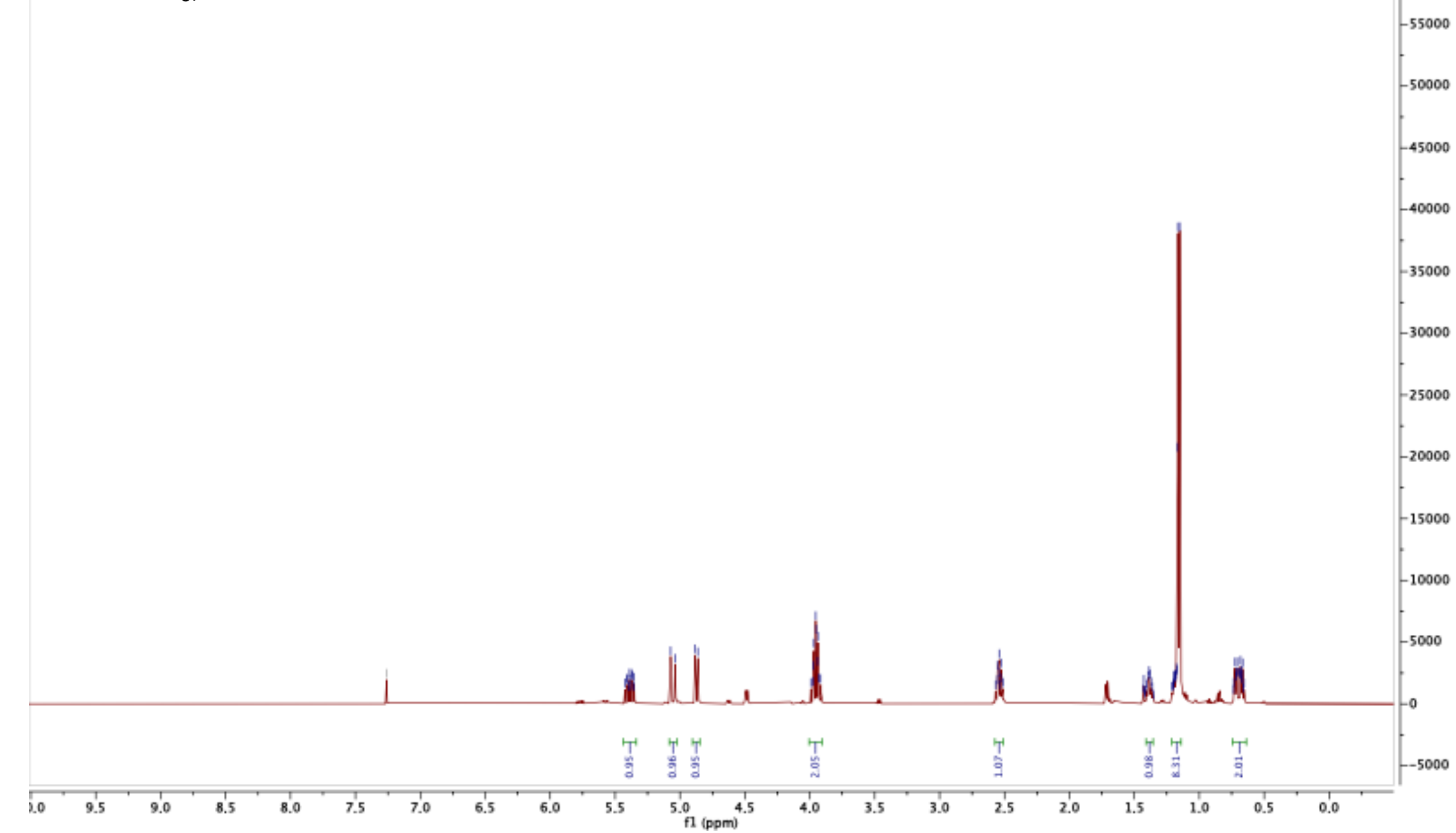

SI-49 


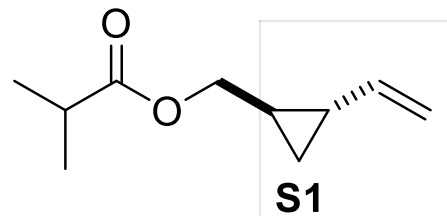

${ }^{13} \mathrm{C}\left\{{ }^{1} \mathrm{H}\right\}$ NMR (126 MHz, $\left.\mathrm{CDCl}_{3}\right)$

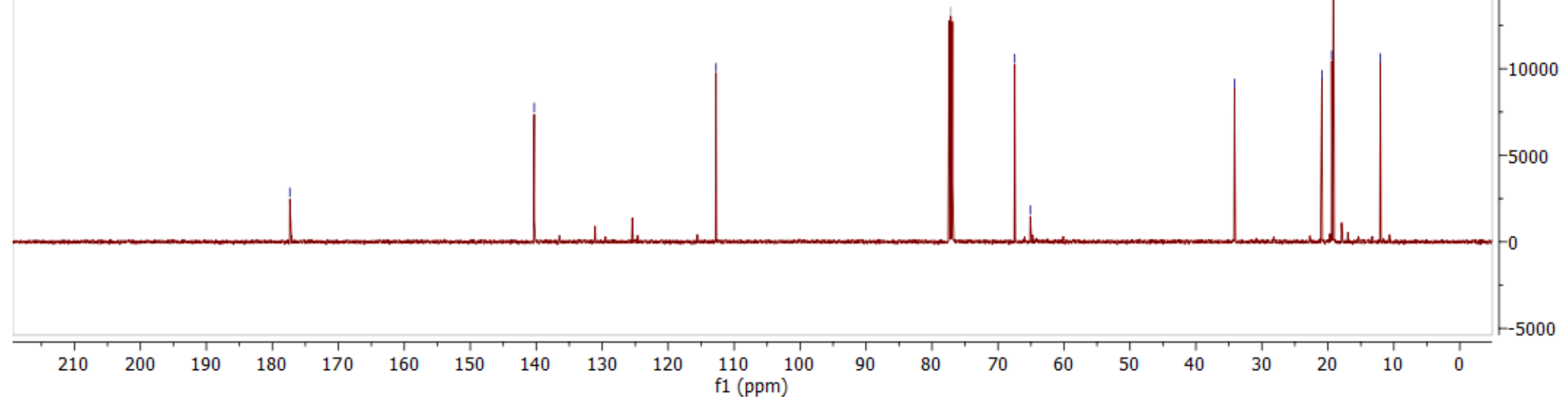




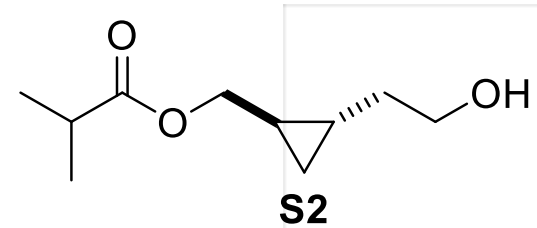

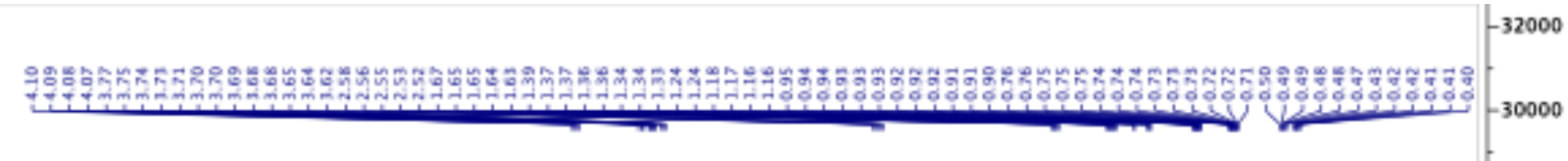

${ }^{1} \mathrm{H}$ NMR $\left(500 \mathrm{MHz}, \mathrm{CDCl}_{3}\right)$

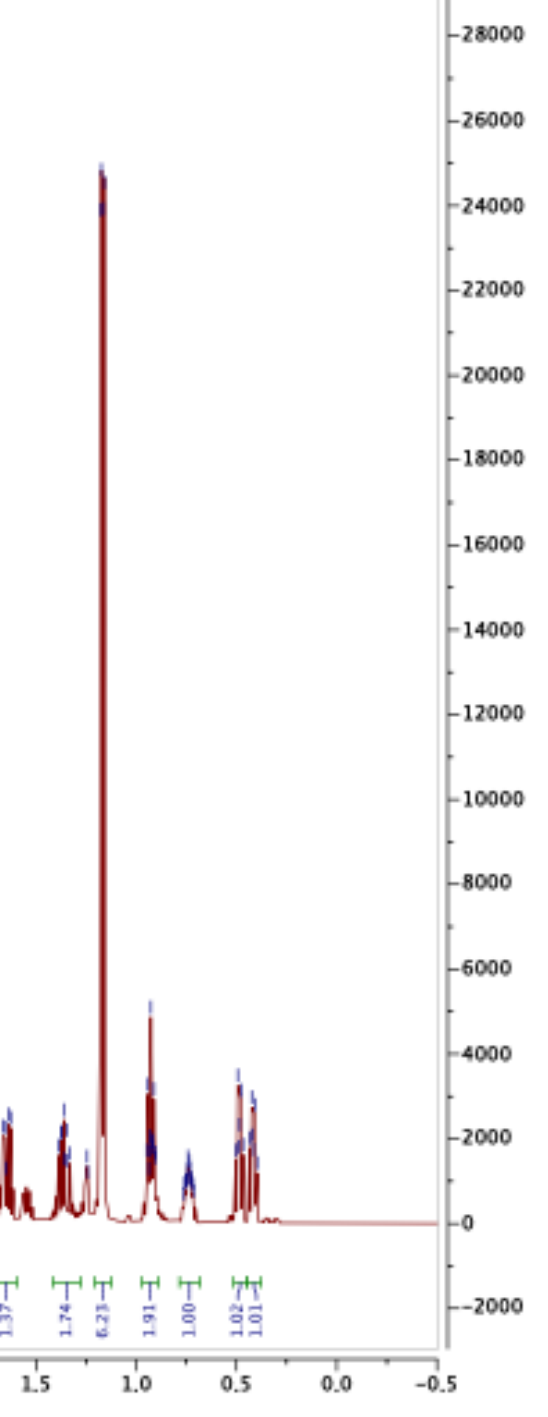

SI-51 


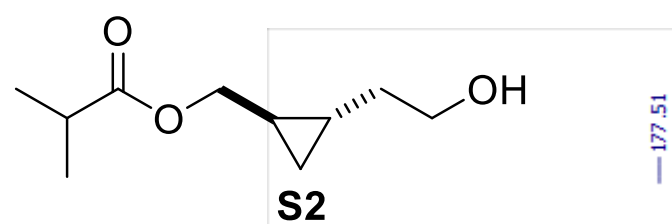

S2

${ }^{13} \mathrm{C}\left\{{ }^{1} \mathrm{H}\right\}$ NMR (126 MHz, $\left.\mathrm{CDCl}_{3}\right)$

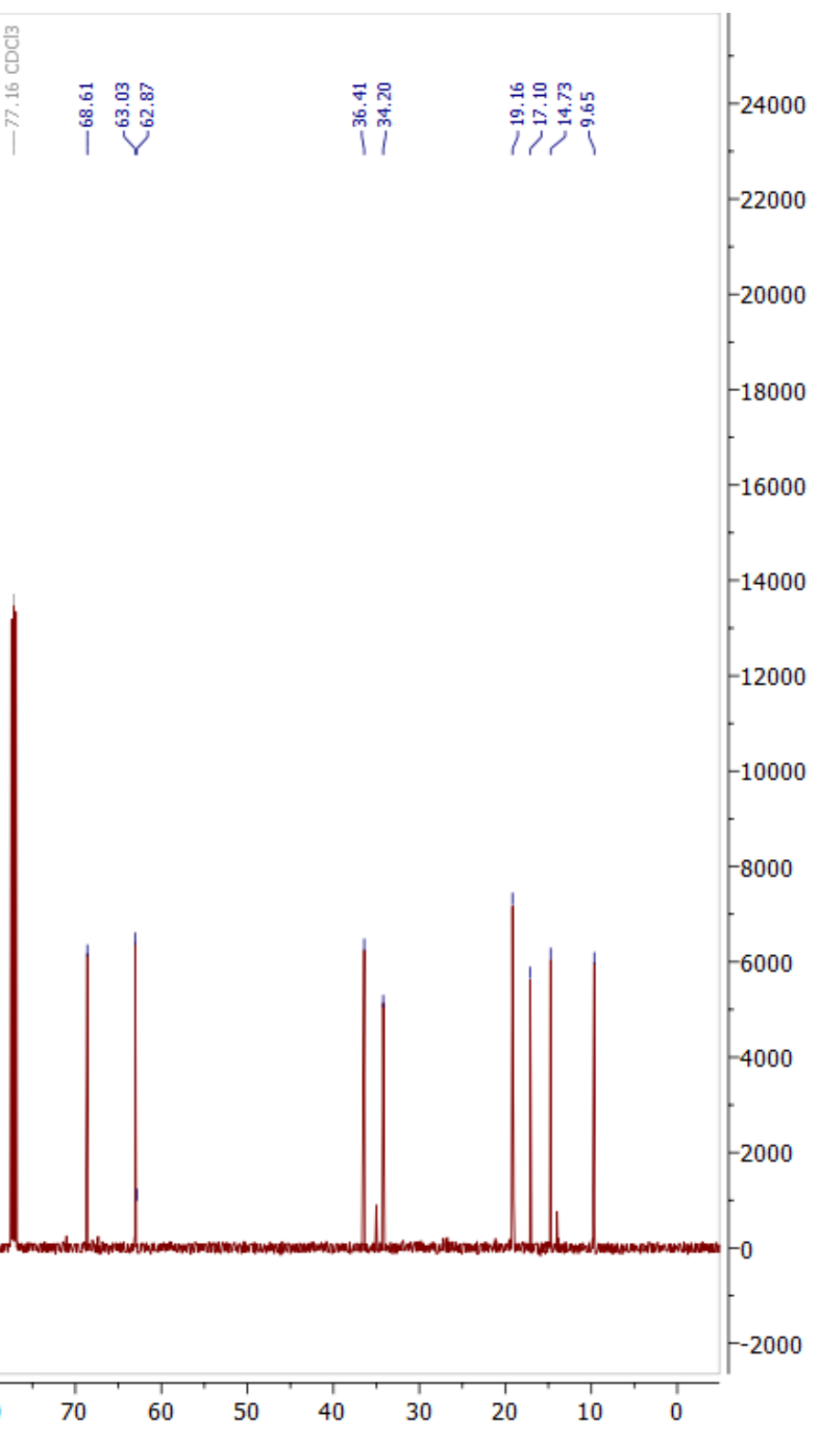

SI-52 


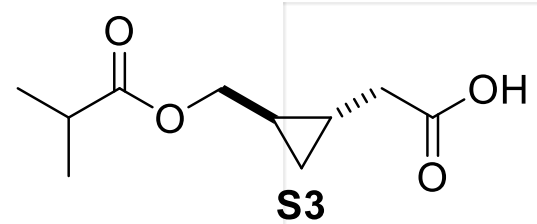

${ }^{1} \mathrm{H}$ NMR $\left(500 \mathrm{MHz}, \mathrm{CDCl}_{3}\right)$

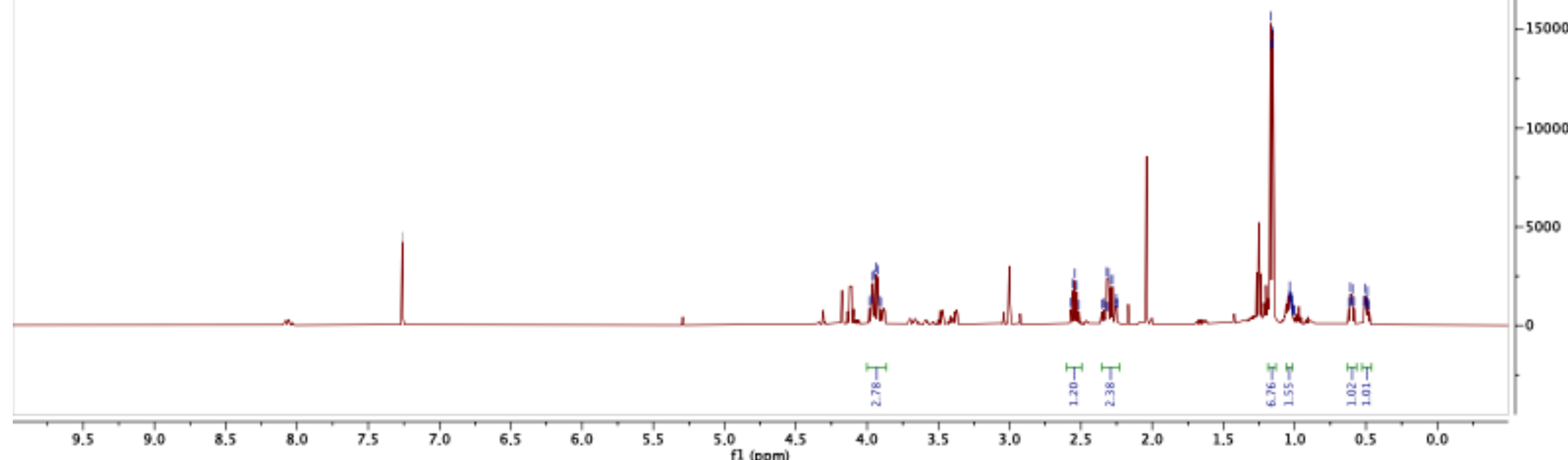




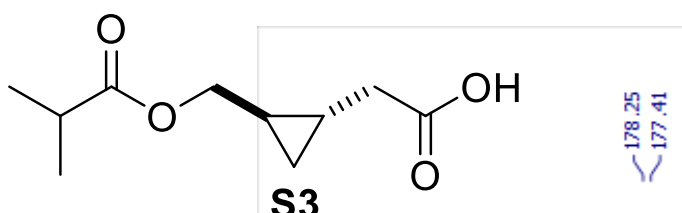

${ }^{13} \mathrm{C}\left\{{ }^{1} \mathrm{H}\right\}$ NMR $\left(126 \mathrm{MHz}, \mathrm{CDCl}_{3}\right)$

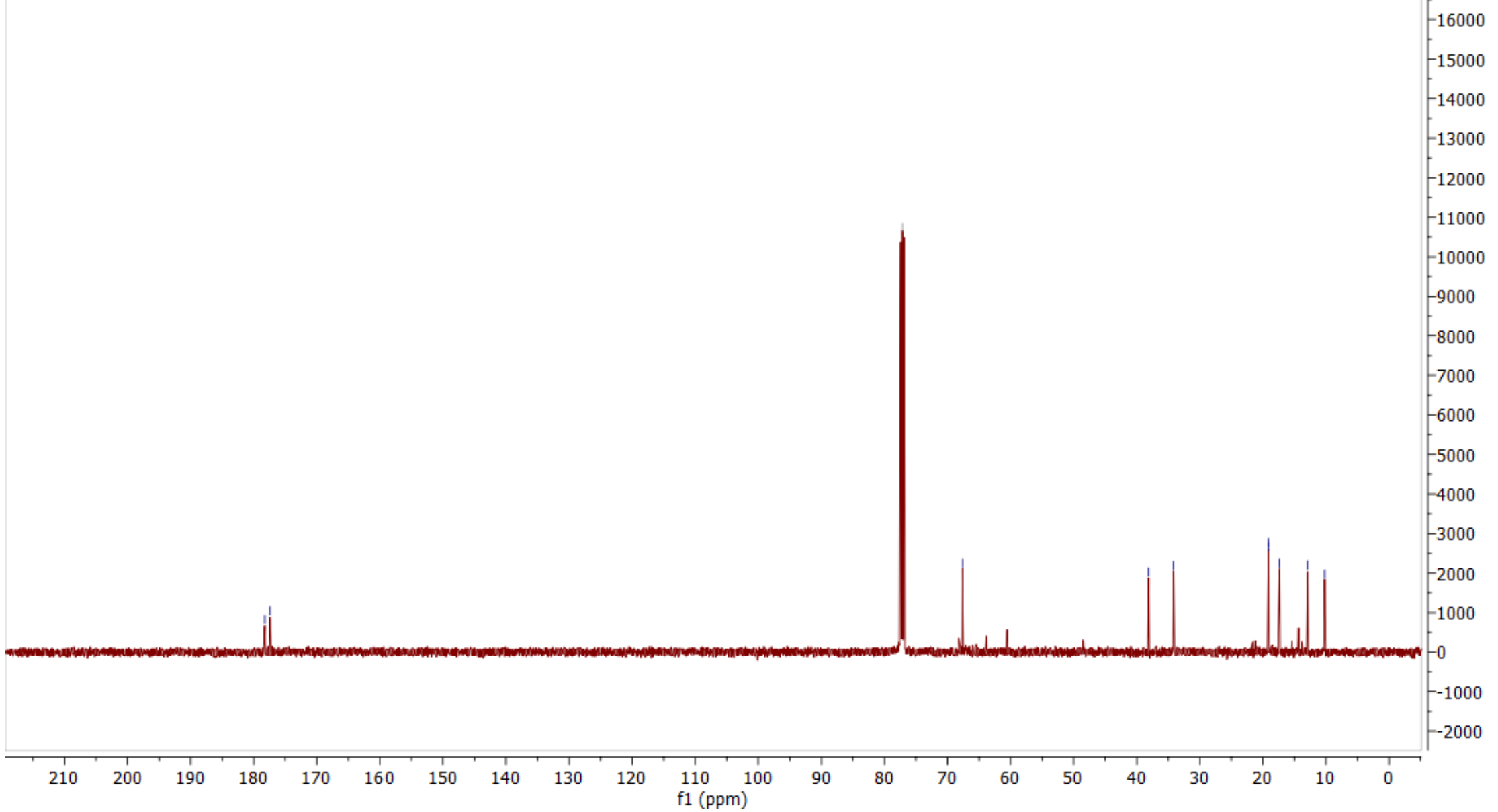




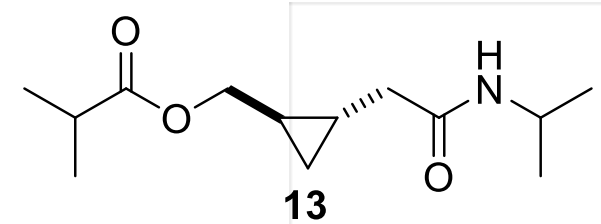

${ }^{1} \mathrm{H}$ NMR $\left(500 \mathrm{MHz}, \mathrm{CDCl}_{3}\right)$

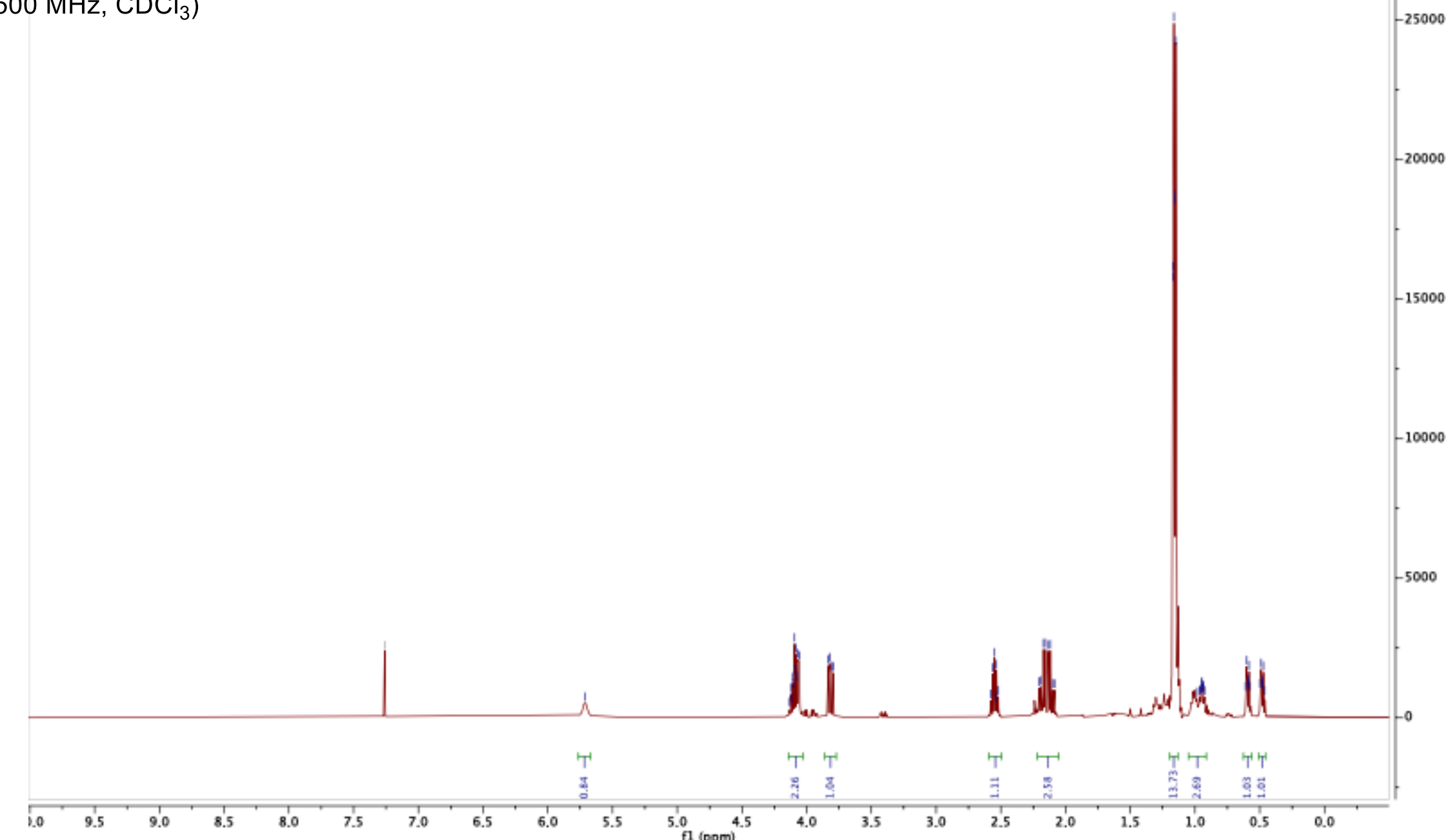




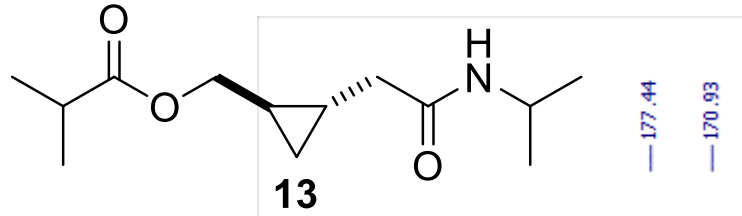

${ }^{13} \mathrm{C}\left\{{ }^{1} \mathrm{H}\right\} \mathrm{NMR}\left(126 \mathrm{MHz}, \mathrm{CDCl}_{3}\right)$
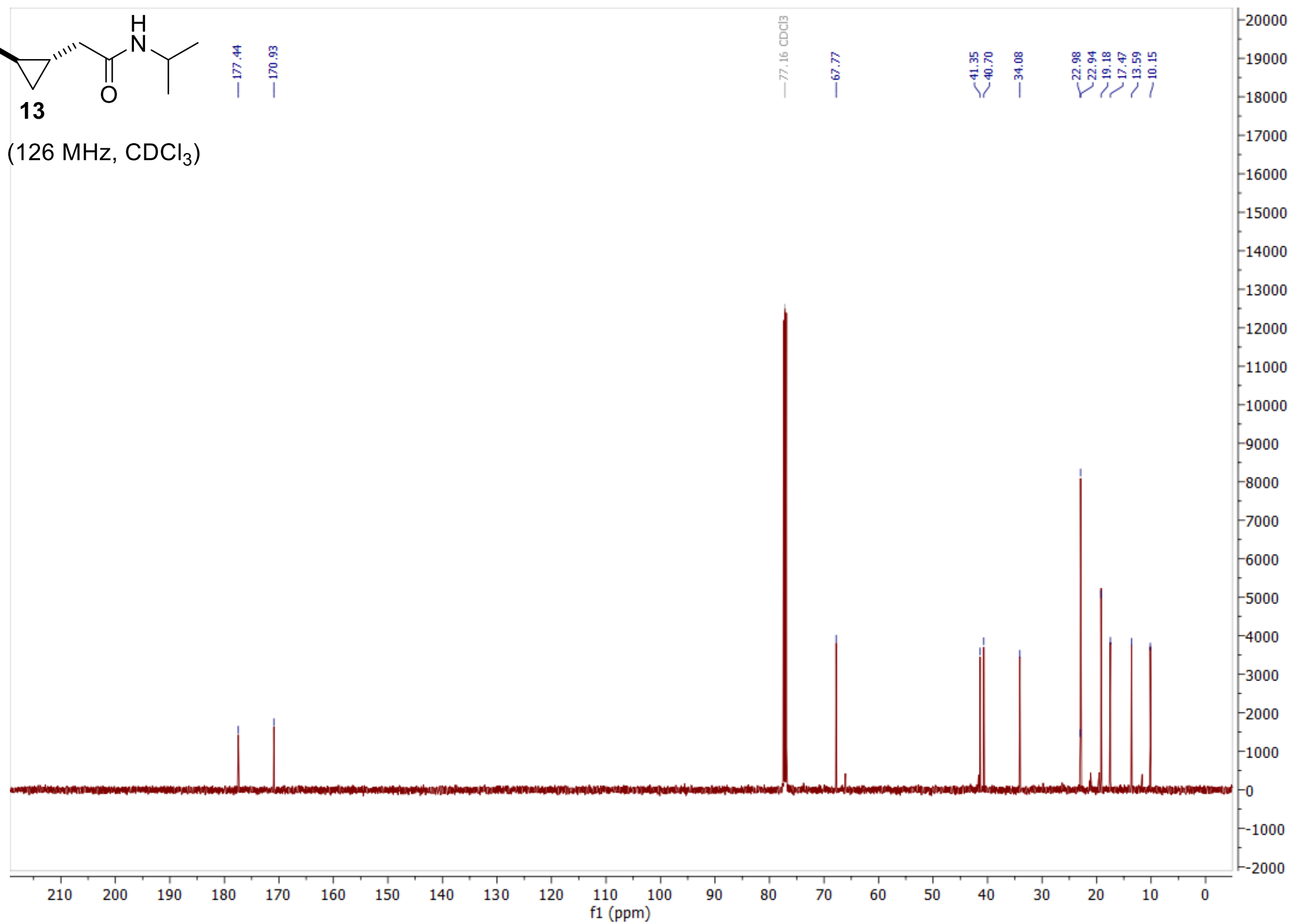

SI-56 


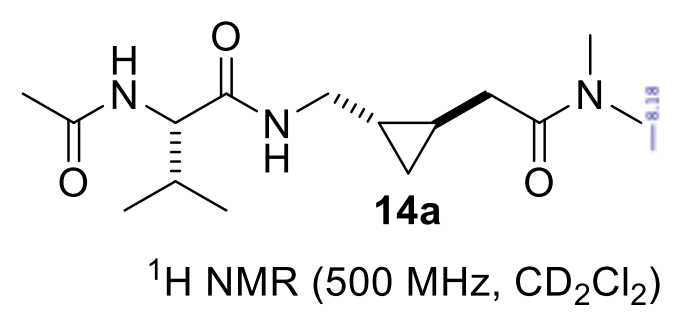

궁
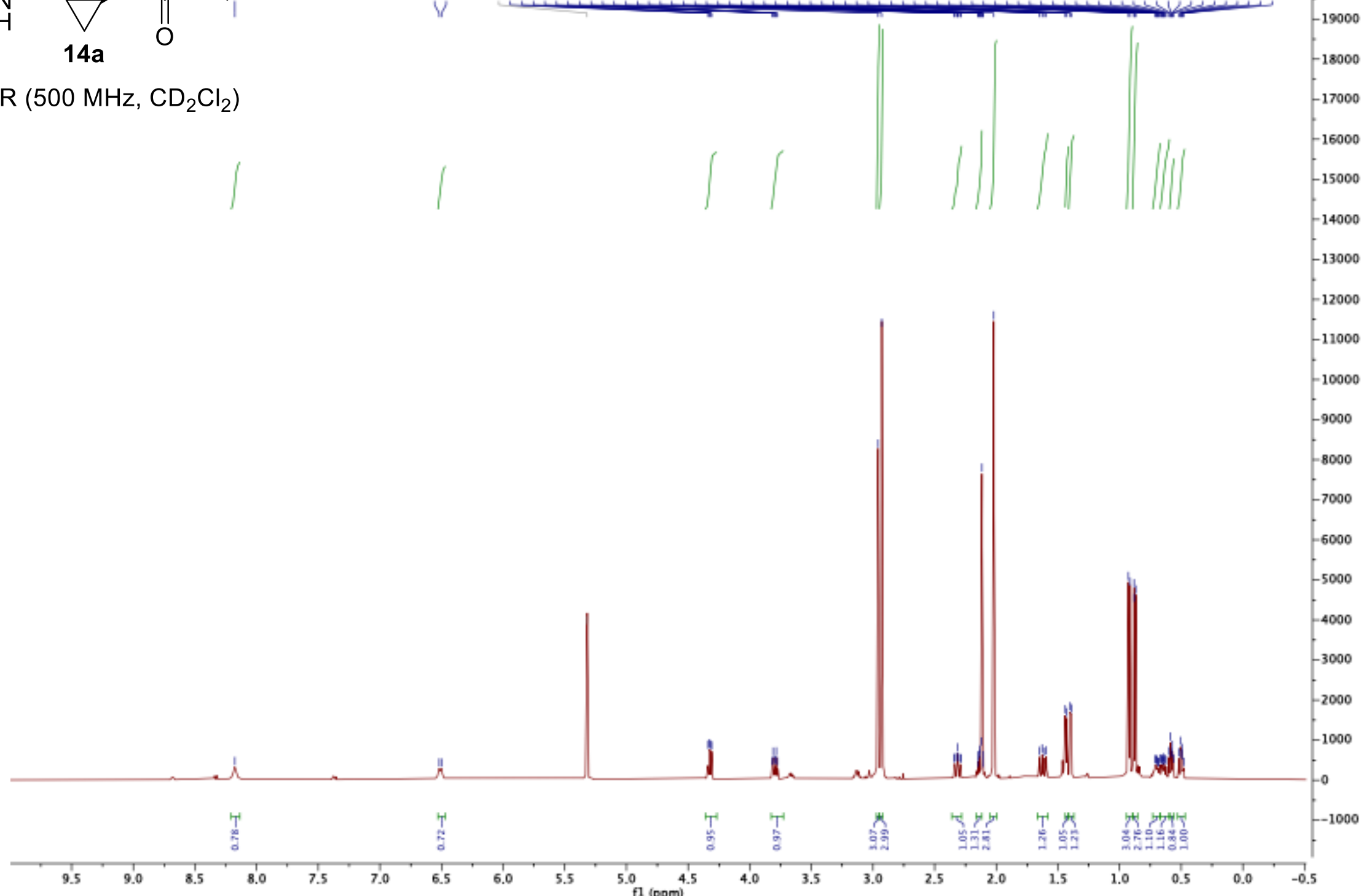

SI-57 


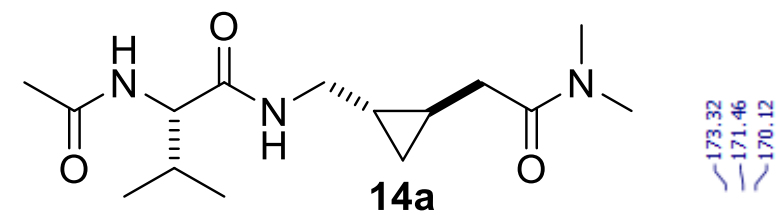

${ }^{13} \mathrm{C}\left\{{ }^{1} \mathrm{H}\right\}$ NMR $\left(126 \mathrm{MHz}, \mathrm{CD}_{2} \mathrm{Cl}_{2}\right)$

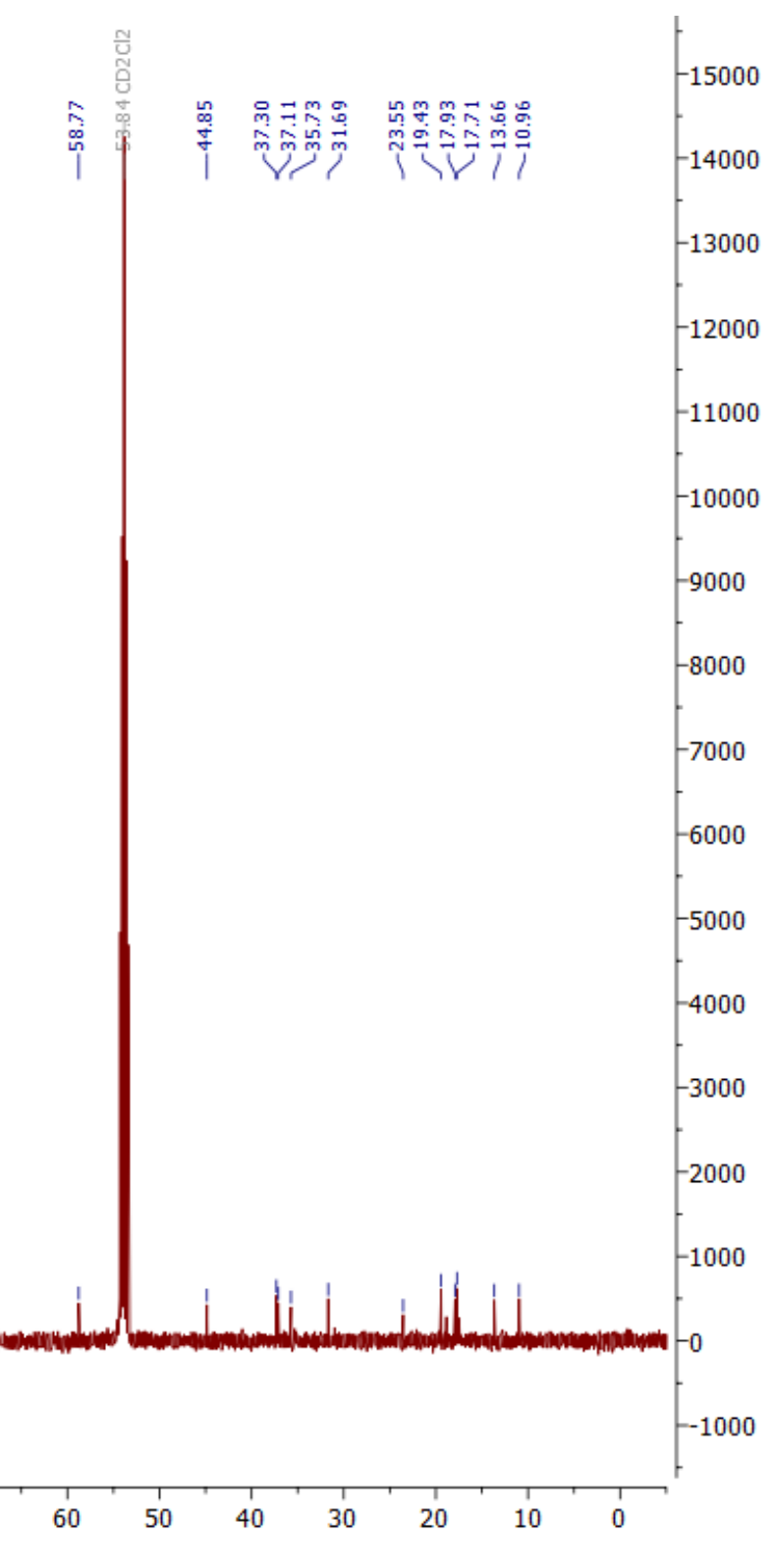

SI-58 


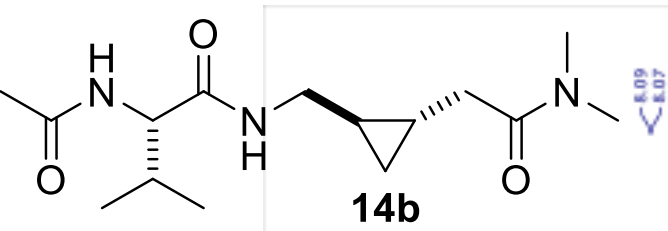

${ }^{1} \mathrm{H}$ NMR $\left(400 \mathrm{MHz}, \mathrm{CDCl}_{3}\right)$

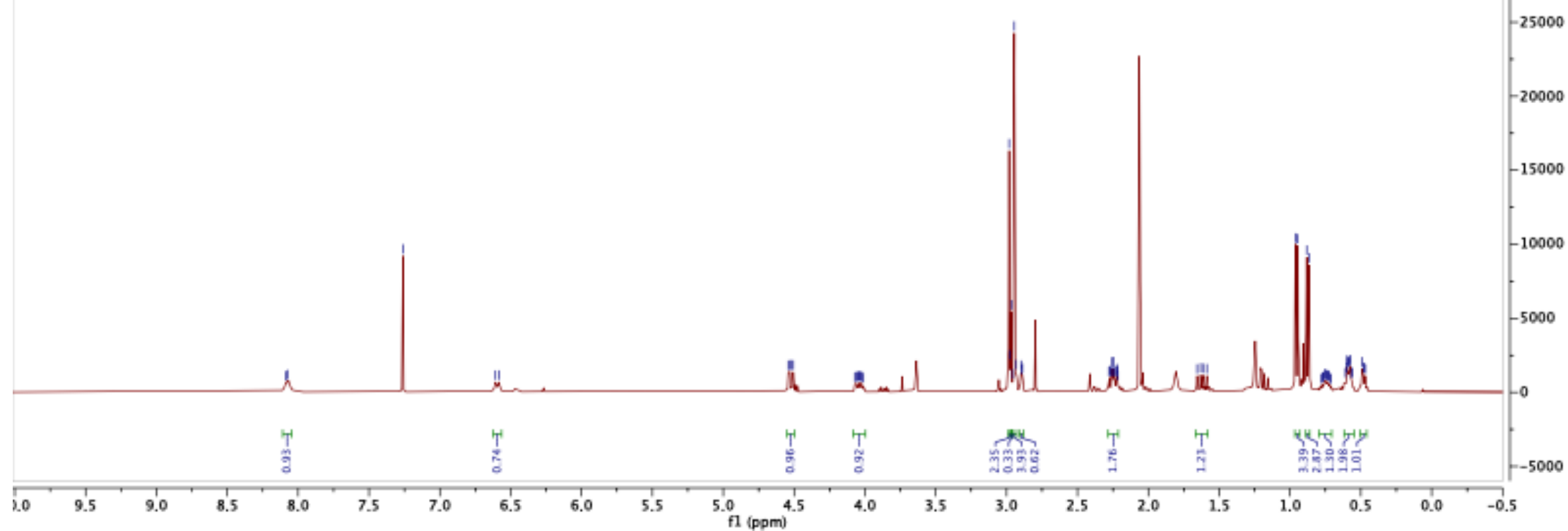

SI-59 

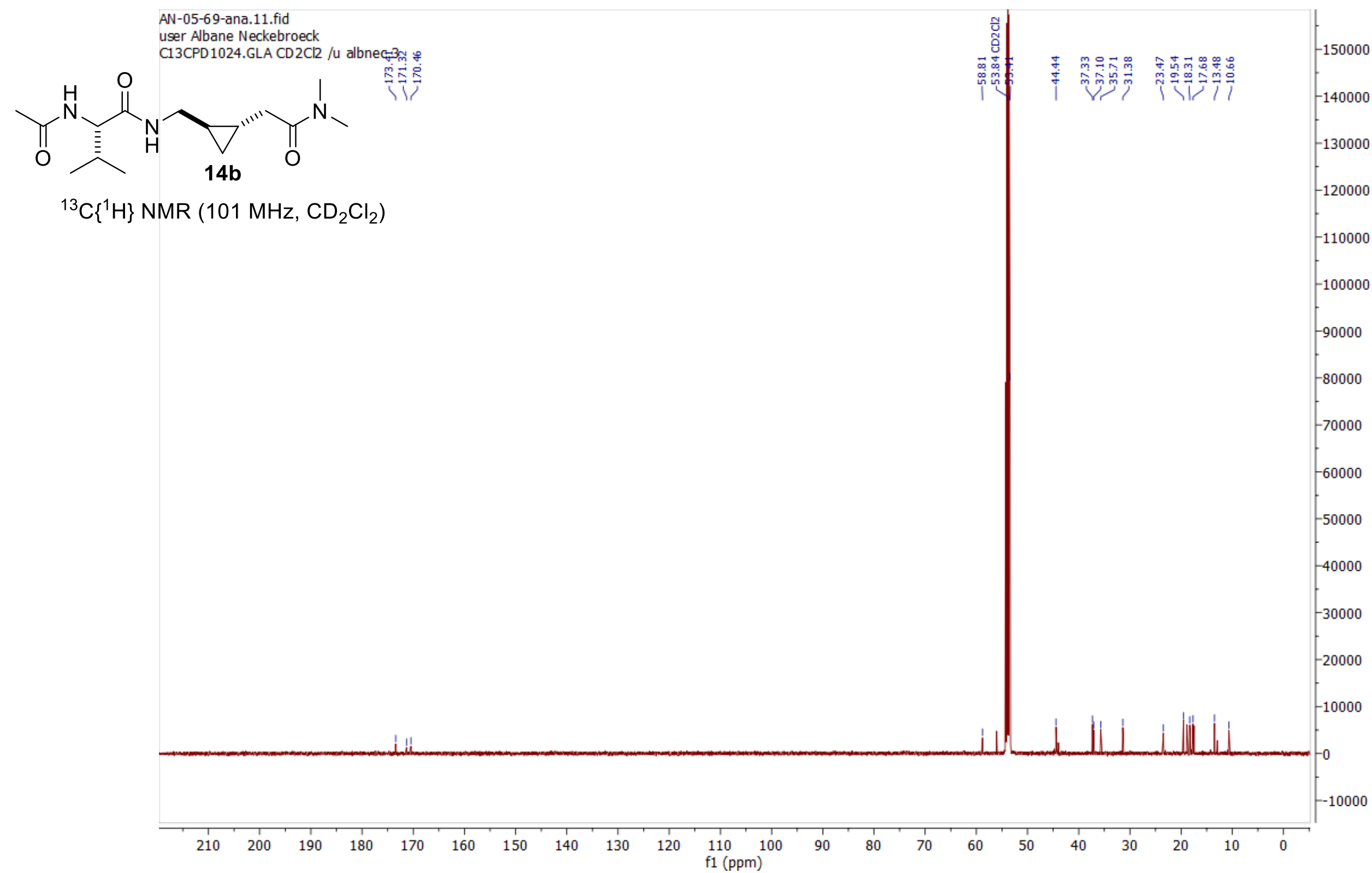

${ }^{13} \mathrm{C}\left\{{ }^{1} \mathrm{H}\right\}$ NMR (101 MHz, $\left.\mathrm{CD}_{2} \mathrm{Cl}_{2}\right)$ 


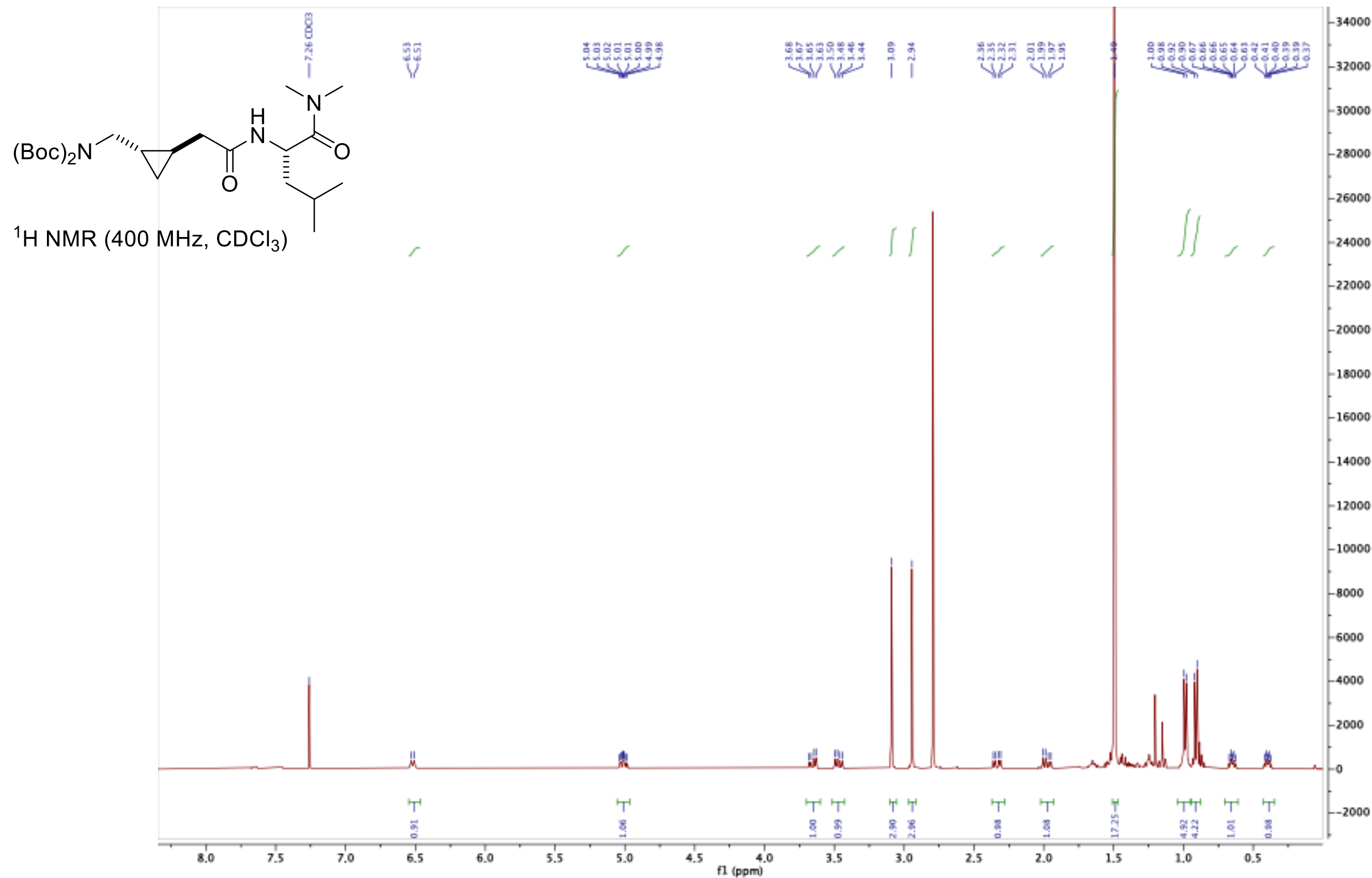

SI-61 


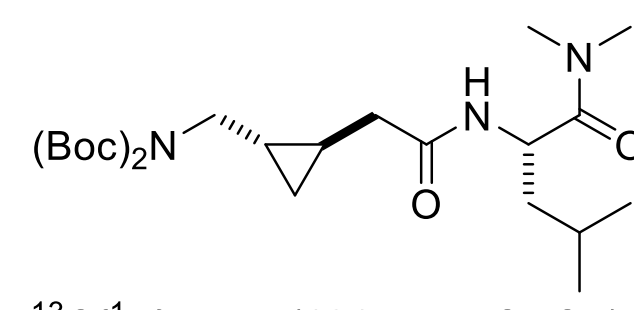

${ }^{13} \mathrm{C}\left\{{ }^{1} \mathrm{H}\right\}$ NMR (101 MHz, CDCl 3 )
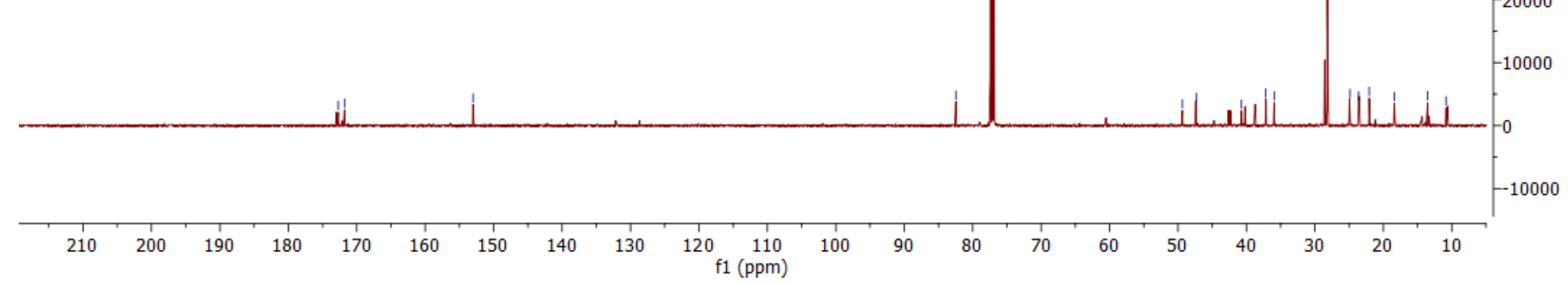


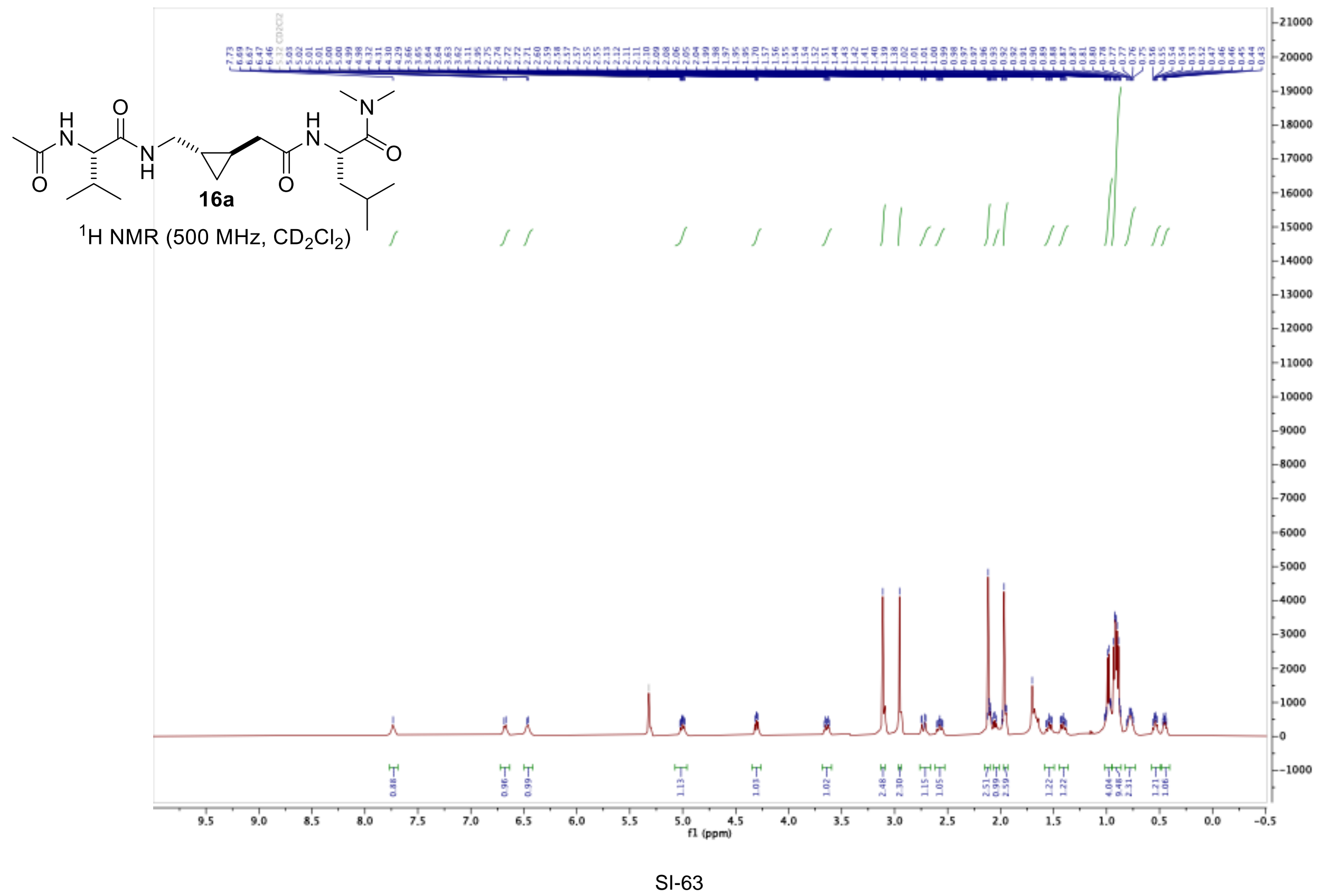



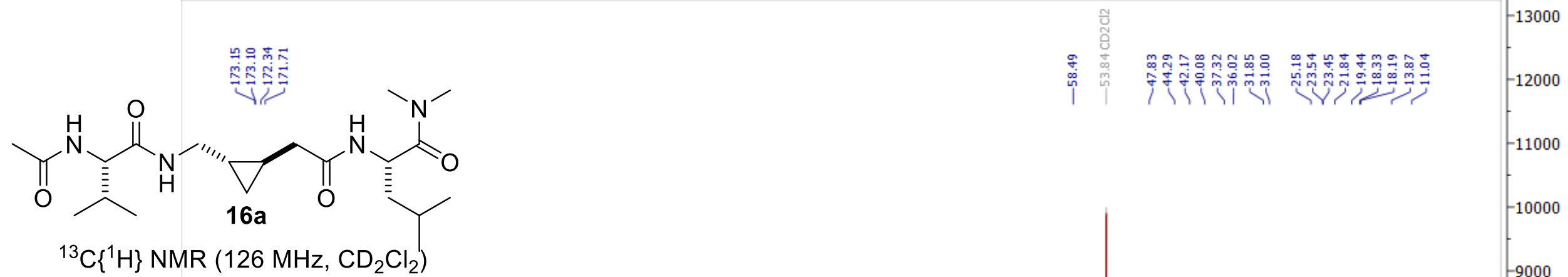

MR (126 MHz, $\mathrm{CD}_{2} \mathrm{Cl}_{2}$ )

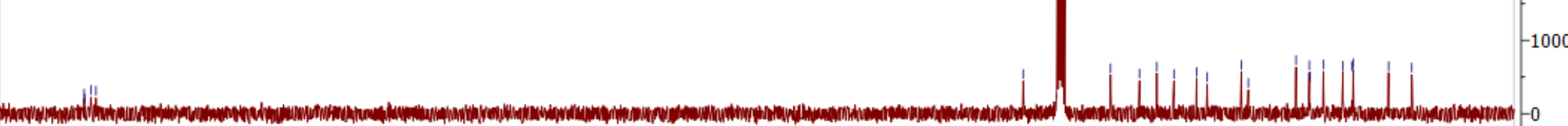

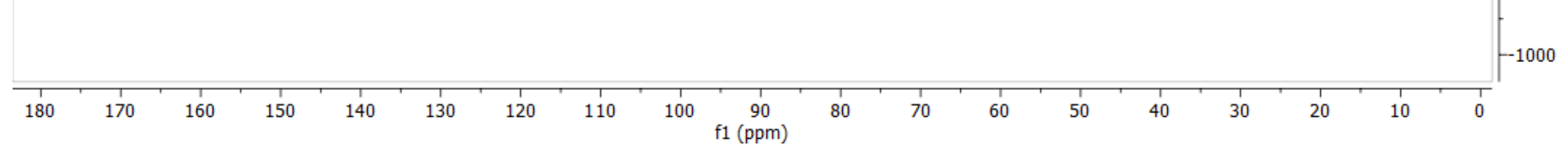

SI-64 

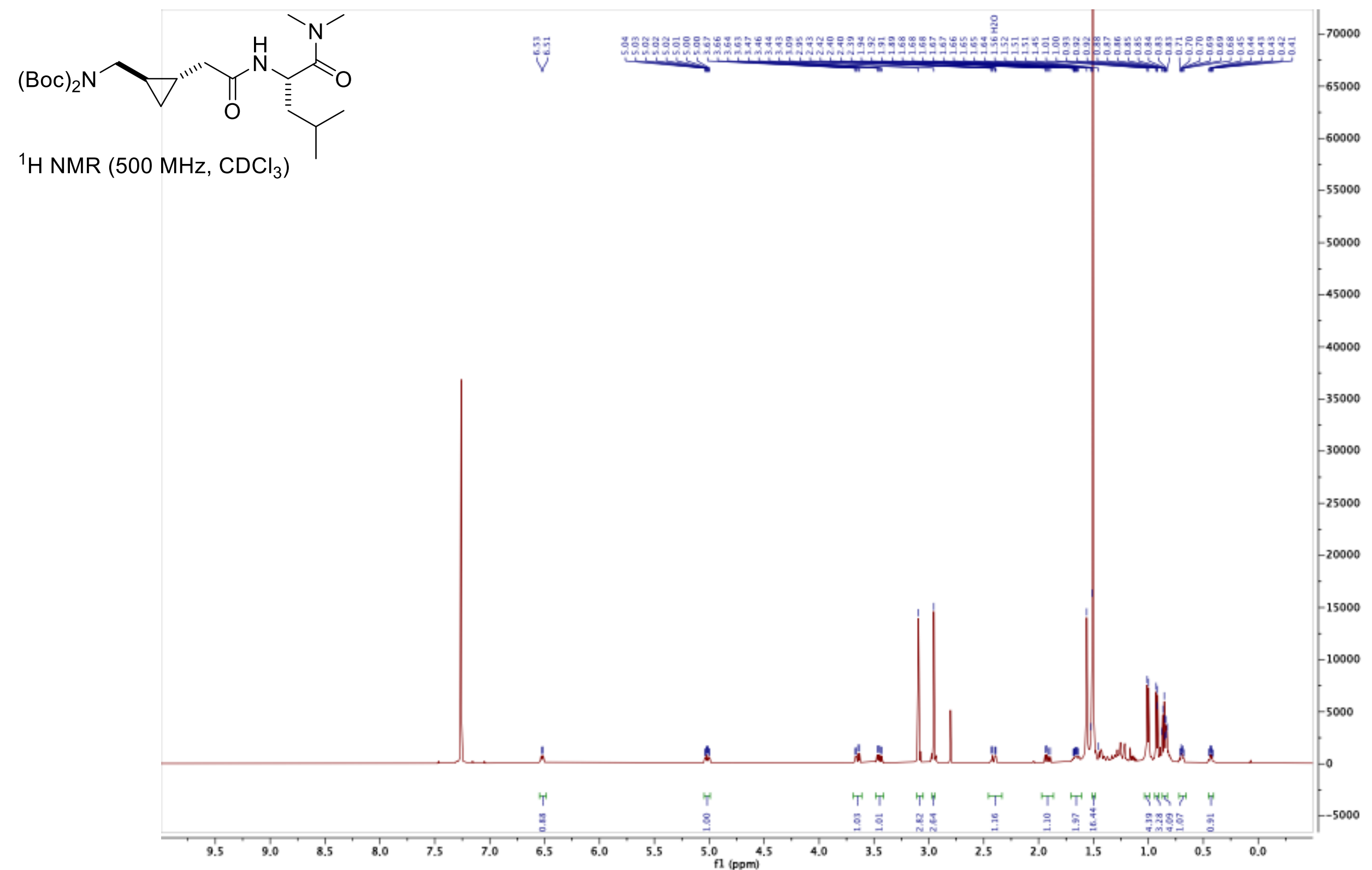

SI-65 


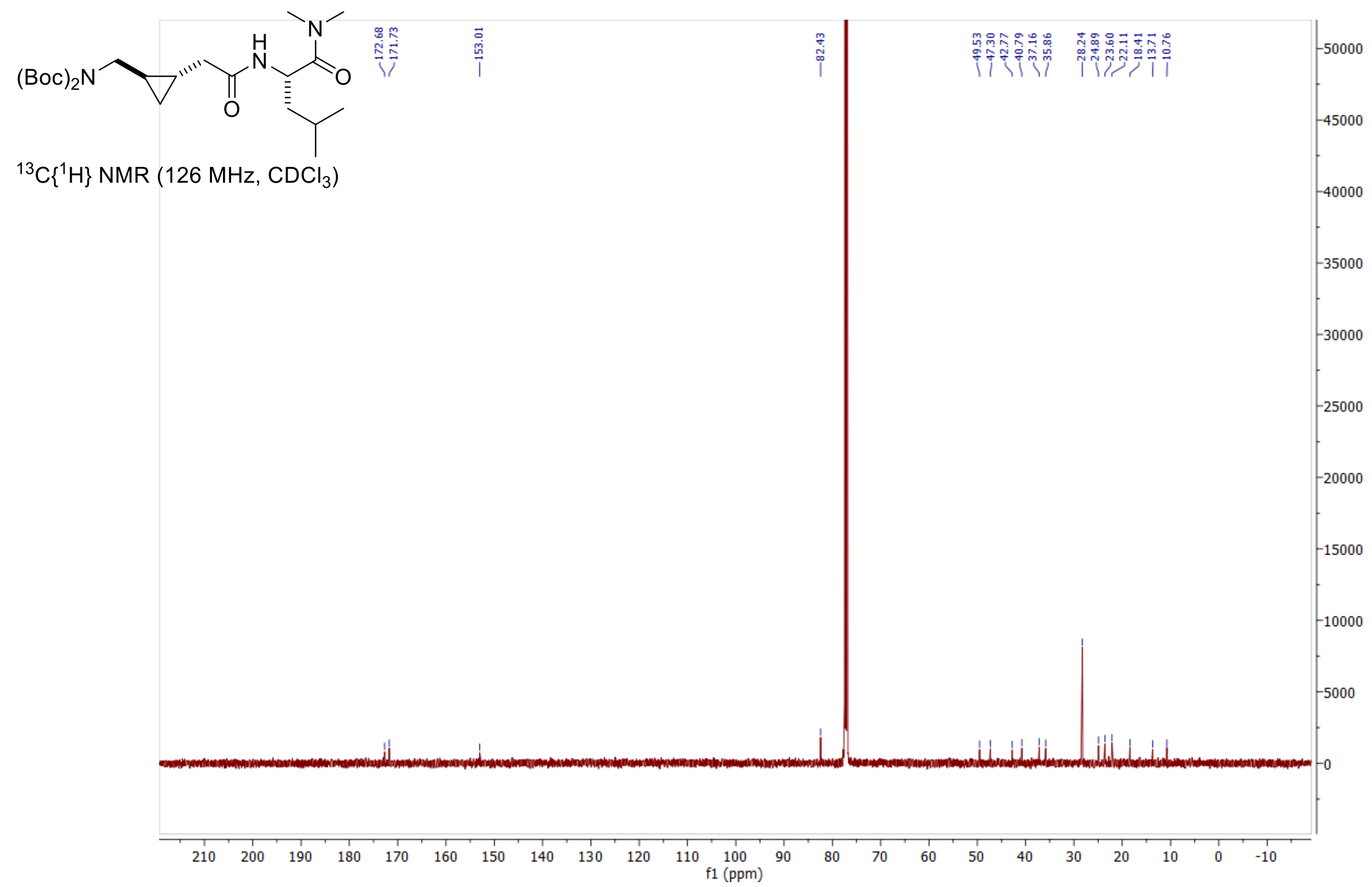




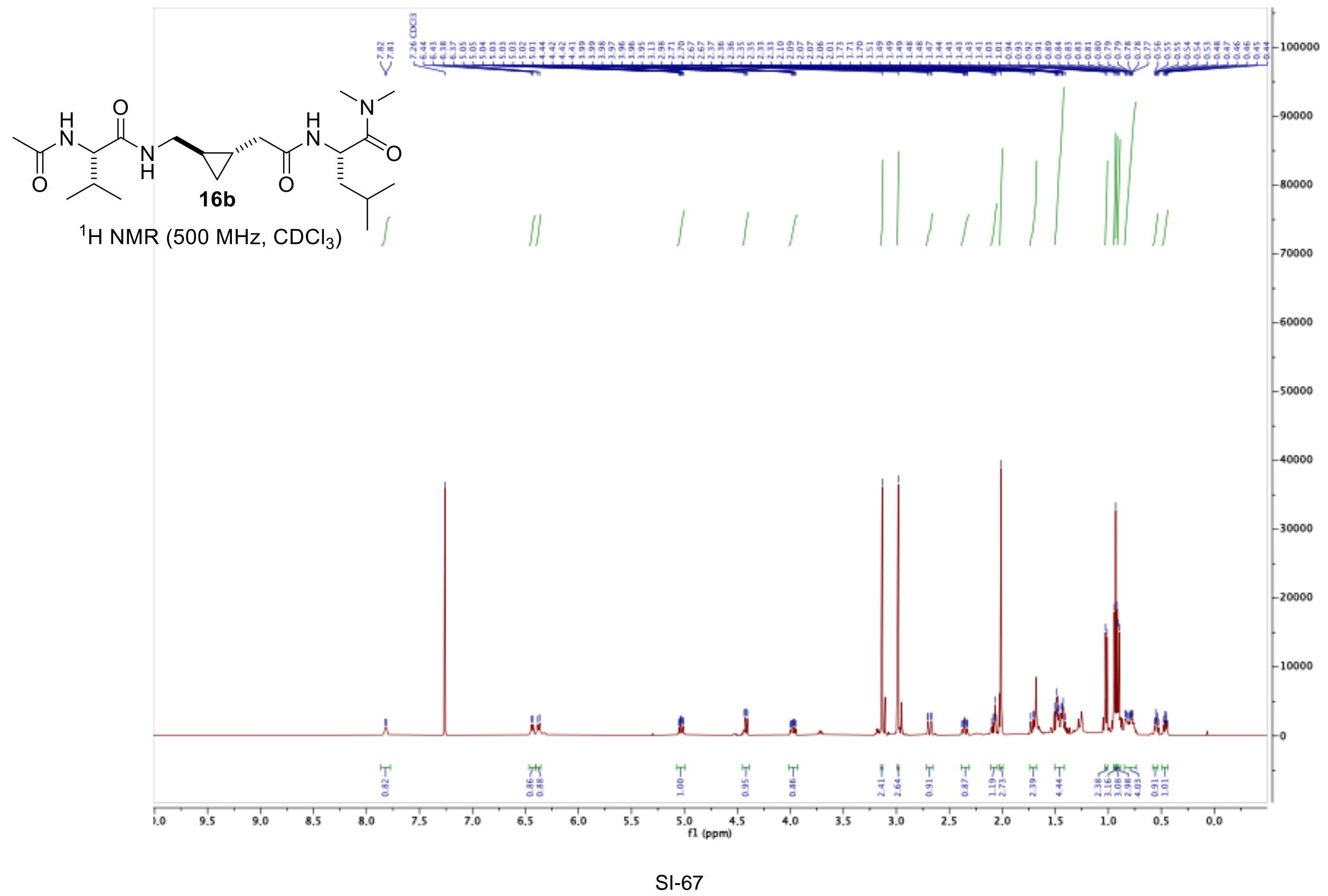




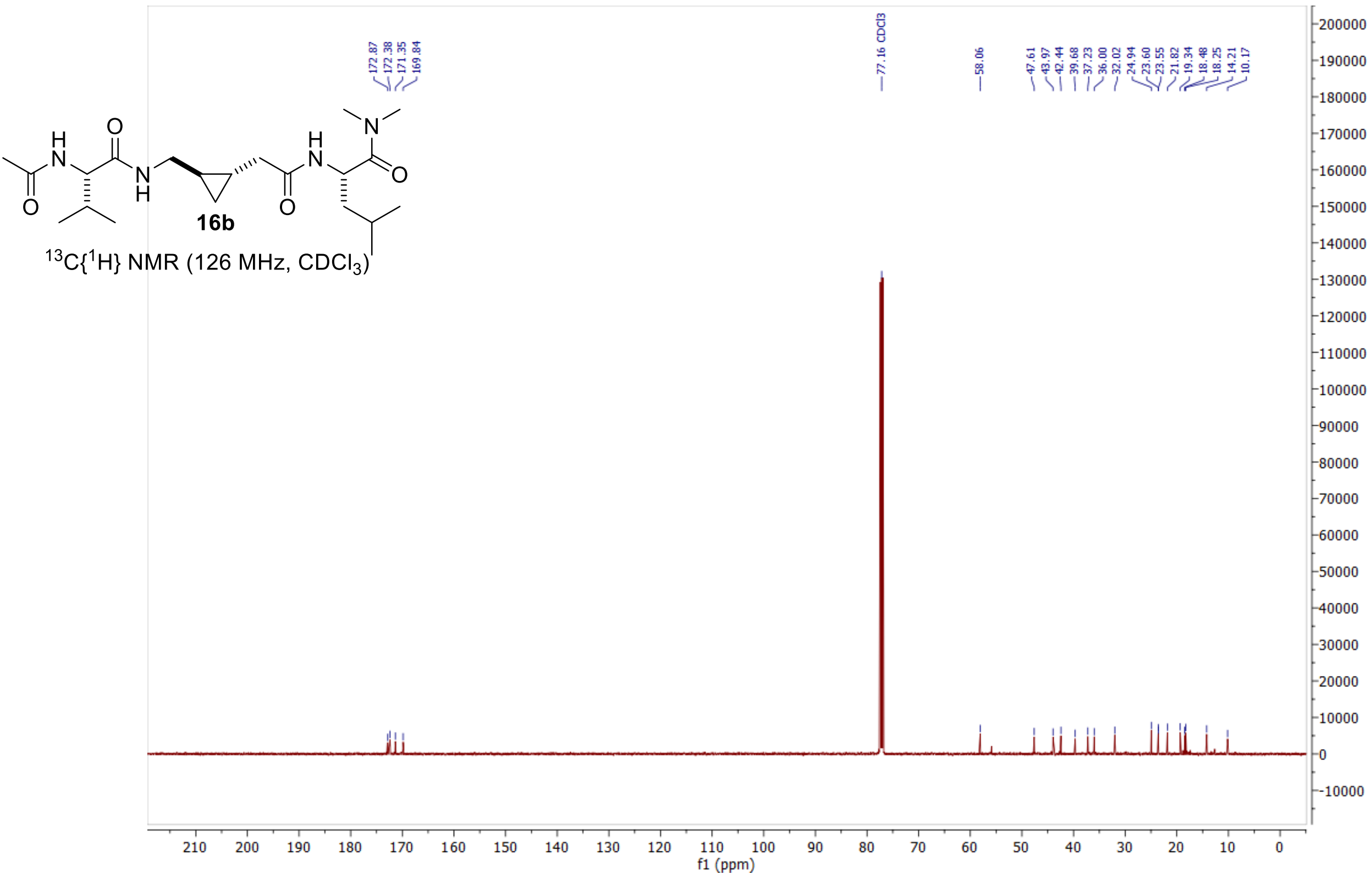

SI-68 


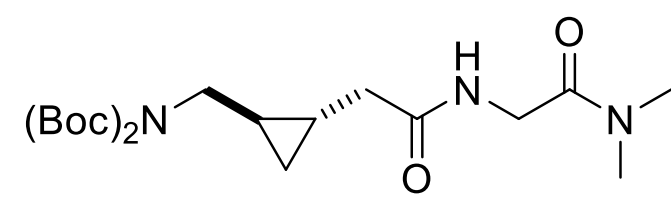

${ }^{1} \mathrm{H}$ NMR $\left(500 \mathrm{MHz}, \mathrm{CDCl}_{3}\right)$

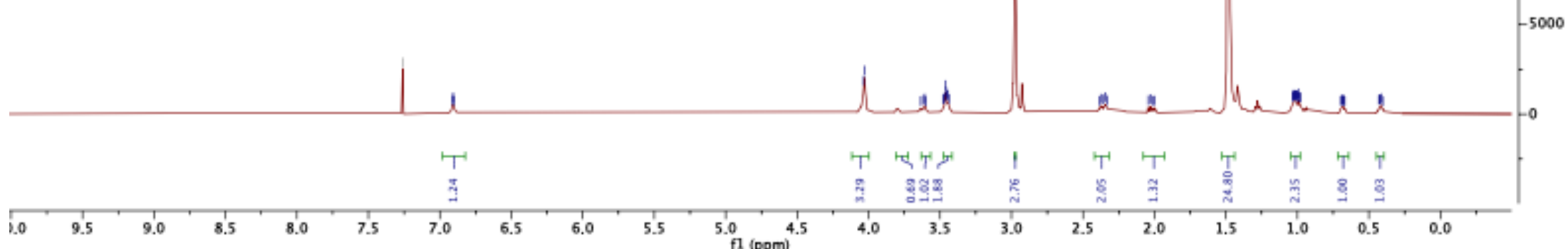




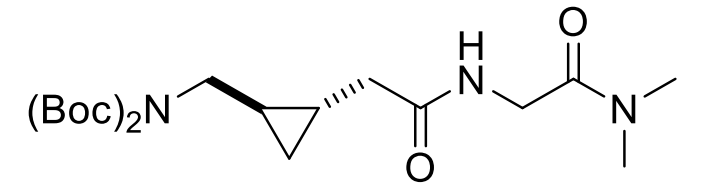

굴

${ }^{13} \mathrm{C}\left\{{ }^{1} \mathrm{H}\right\}$ NMR $\left(126 \mathrm{MHz}, \mathrm{CDCl}_{3}\right)$
$-12000$

11000

10000

9000

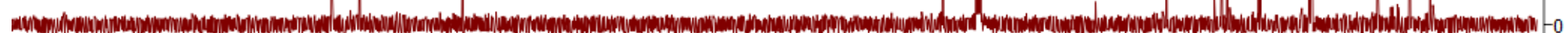




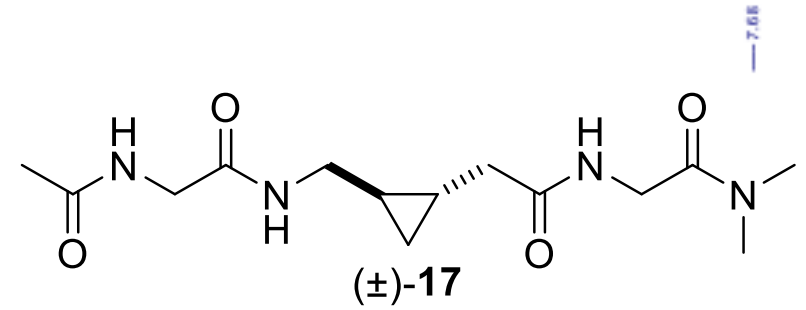

${ }^{1} \mathrm{H}$ NMR $\left(400 \mathrm{MHz}, \mathrm{CDCl}_{3}\right)$

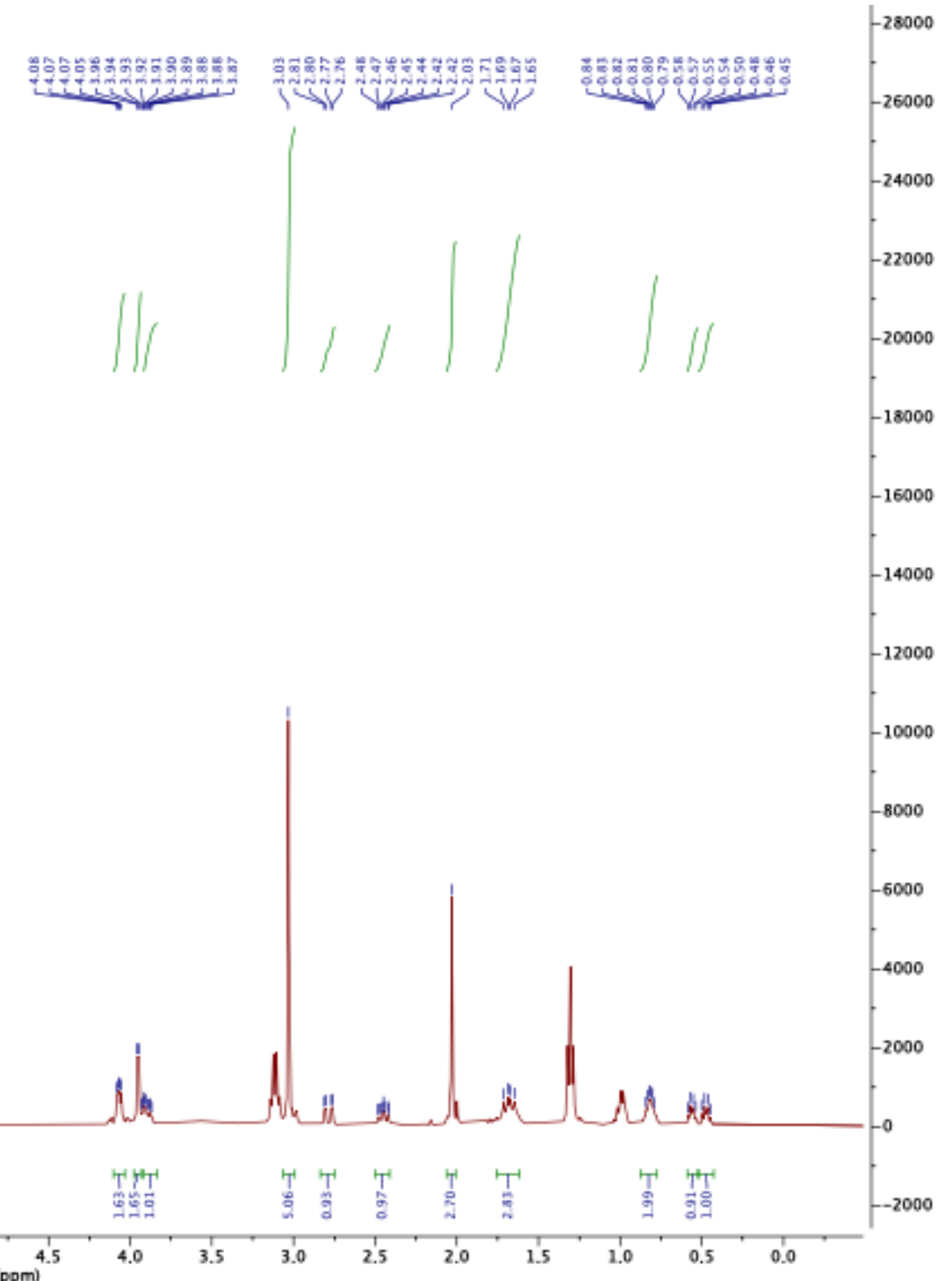

SI-71 

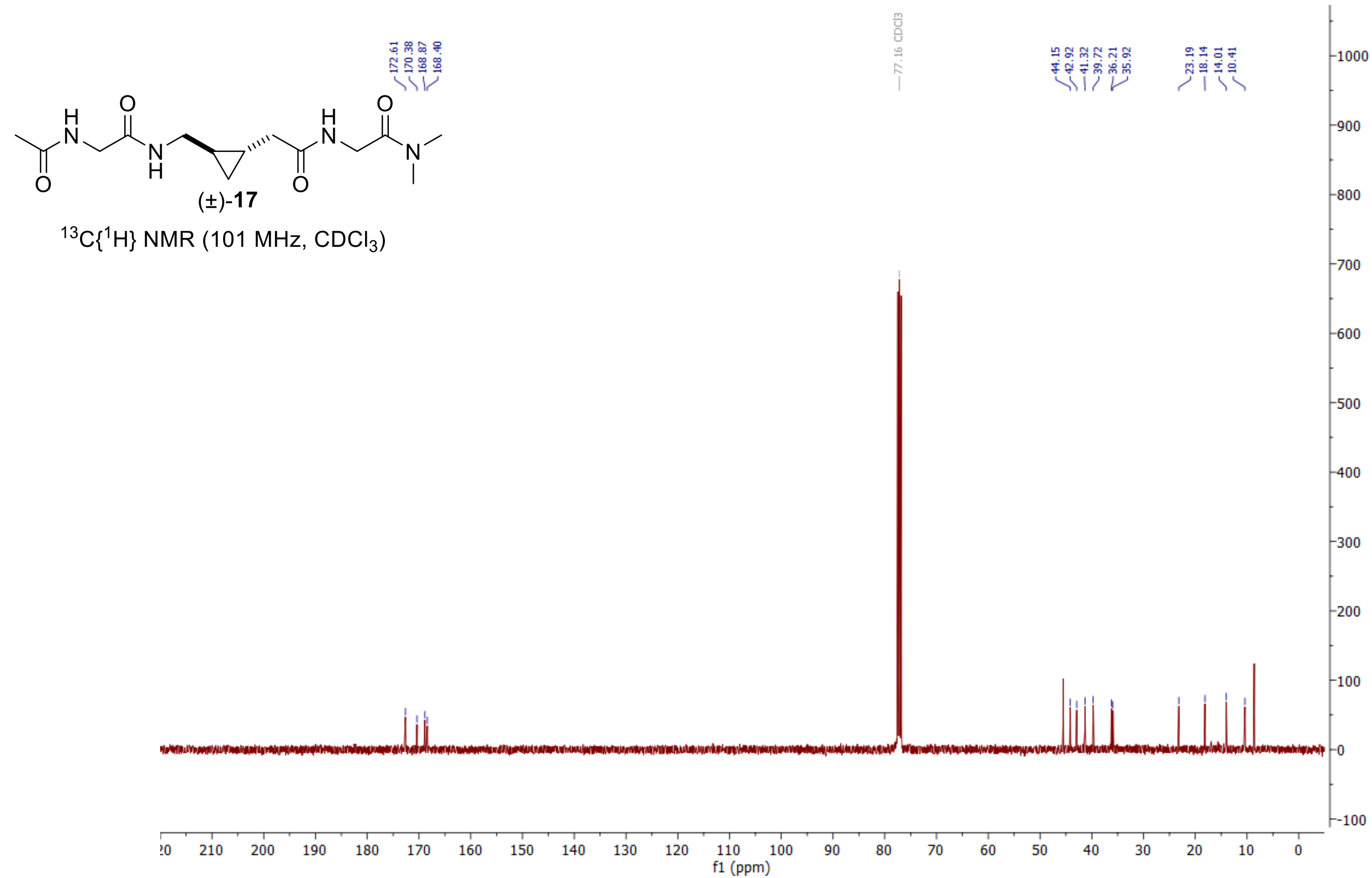

${ }^{13} \mathrm{C}\left\{{ }^{1} \mathrm{H}\right\}$ NMR $\left(101 \mathrm{MHz}, \mathrm{CDCl}_{3}\right)$ 


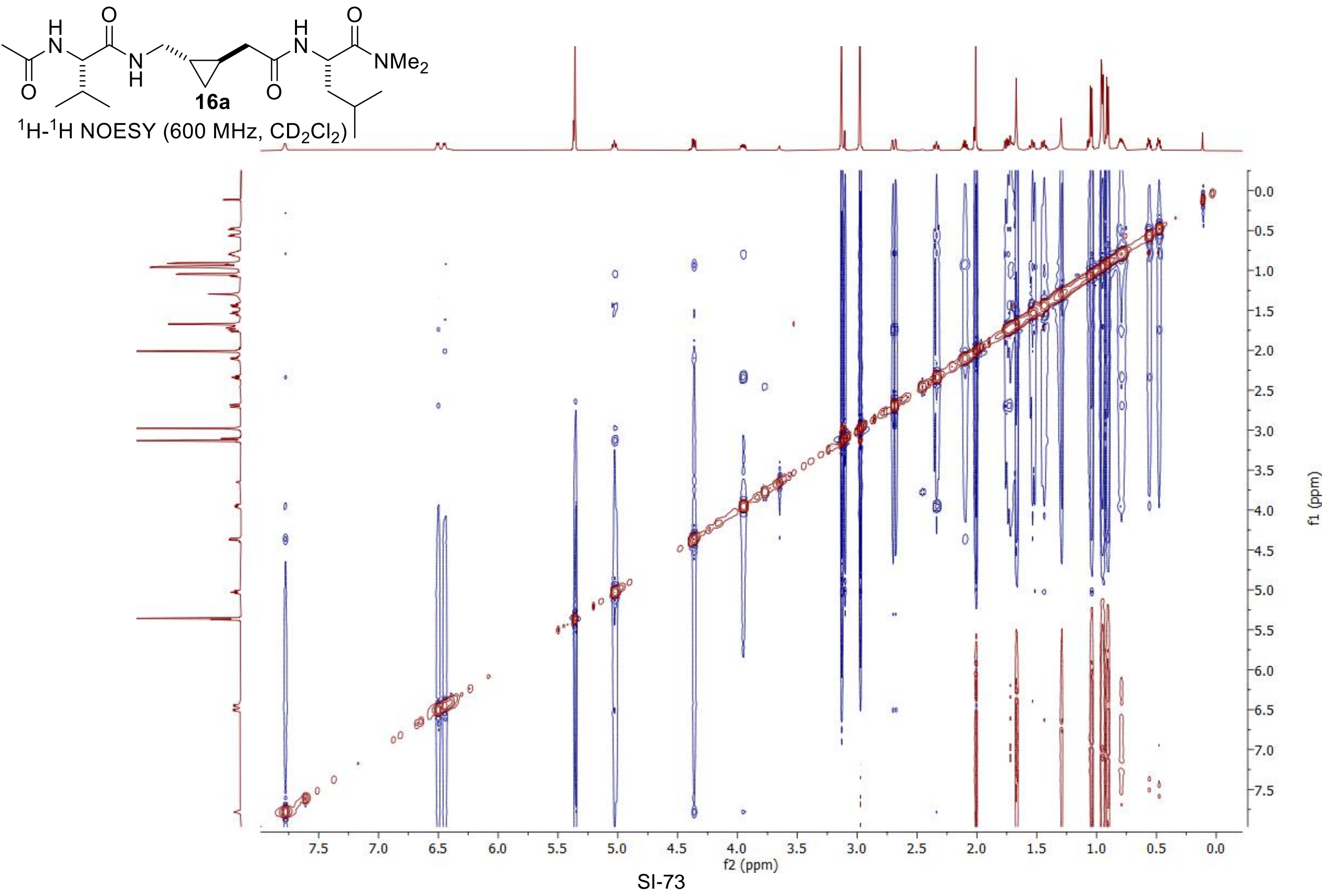



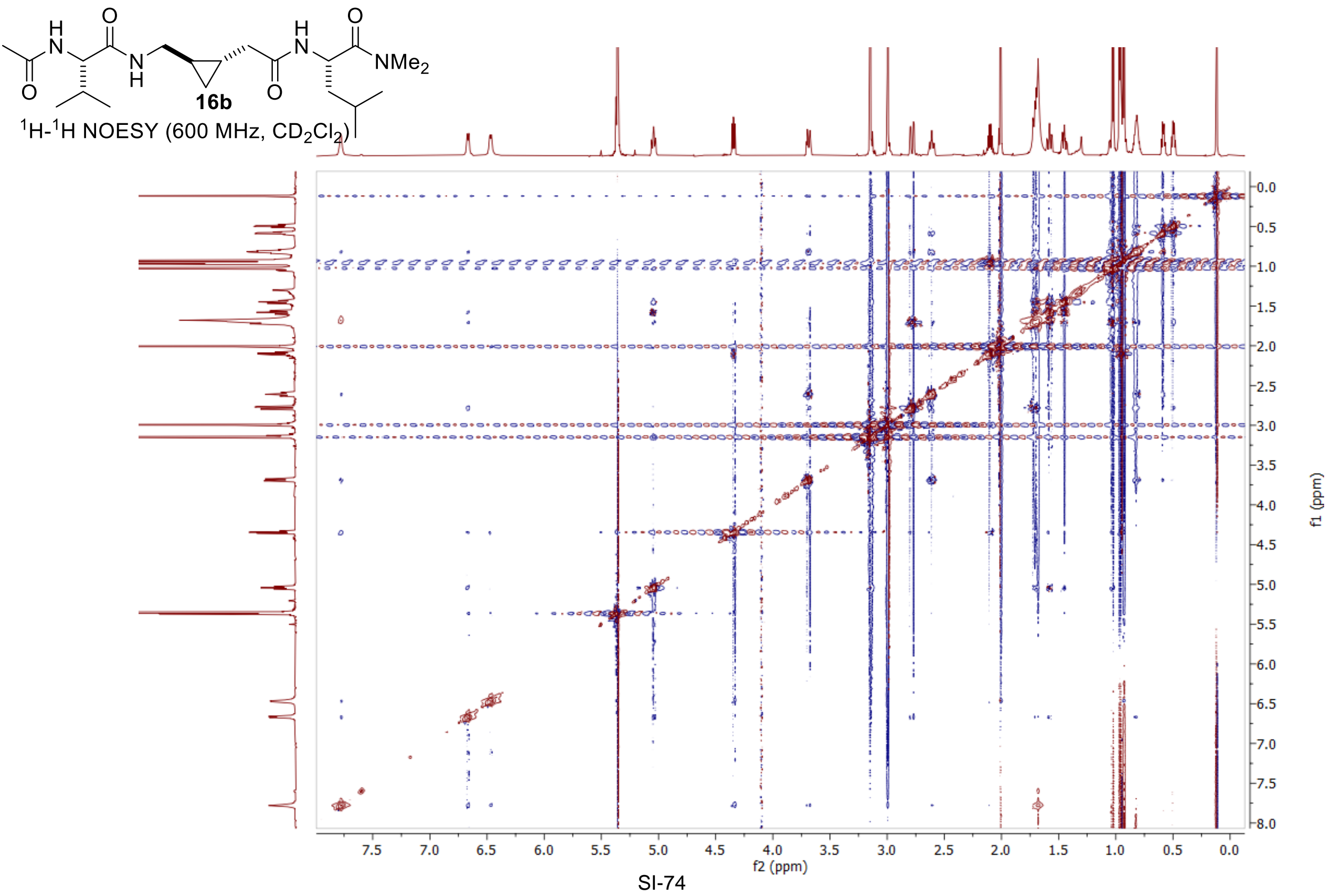
Concentration-Dependent NMR Data (Figures 3, 5 and 6)
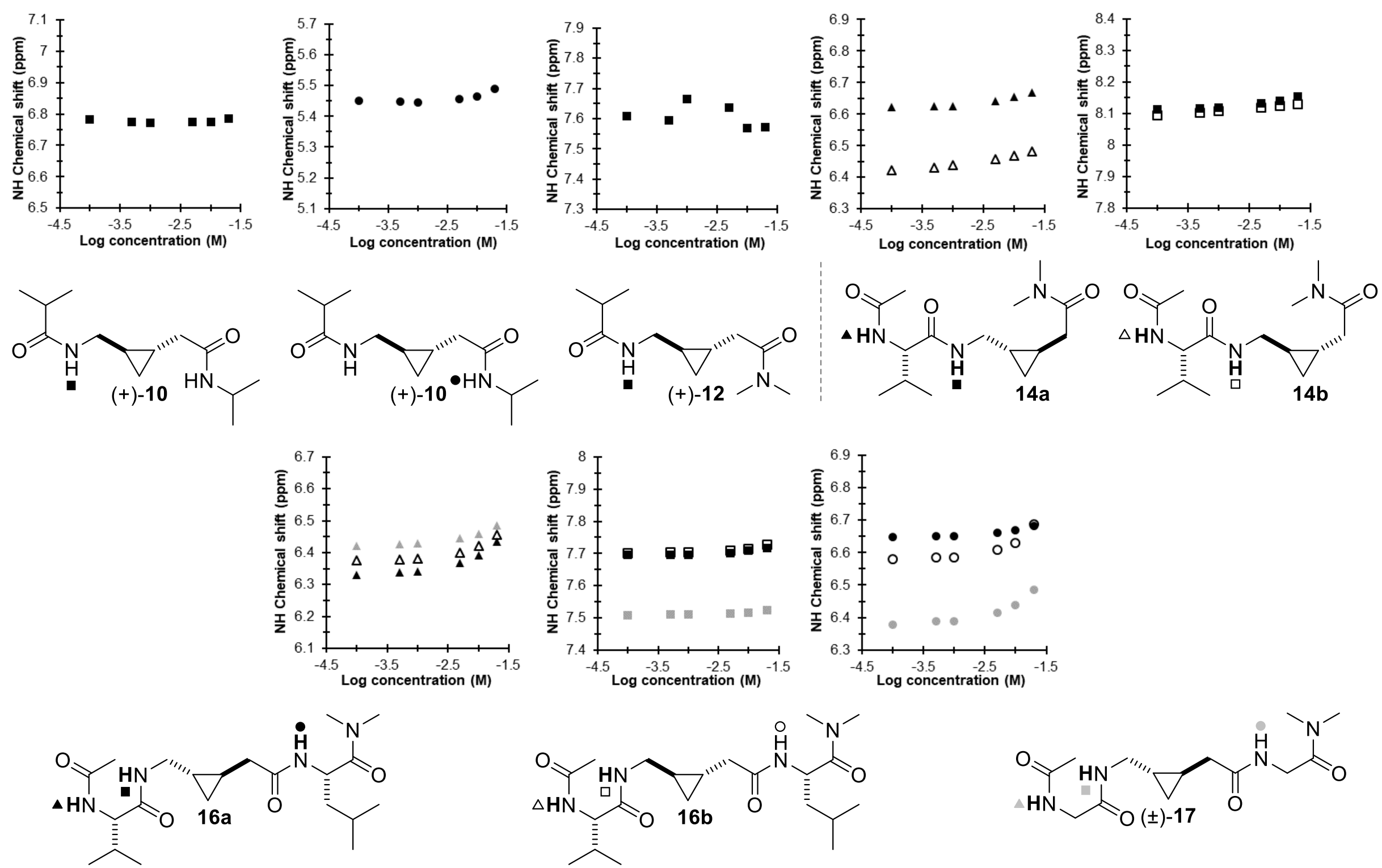


\section{Amide N-H IR Data at a Concentration of $10 \mathrm{mM}$ (Figures 3, 5 and 6)}
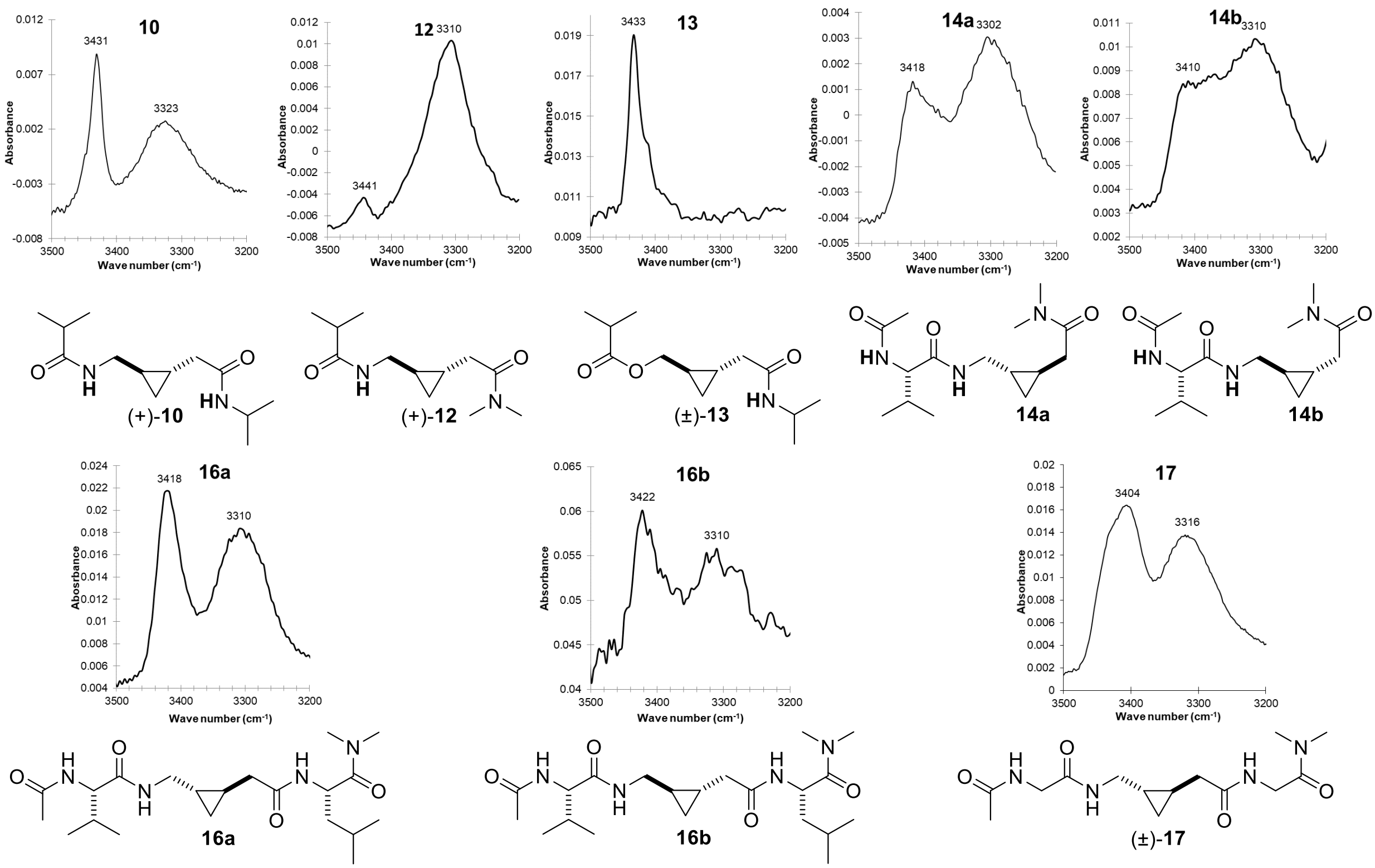


\section{Circular Dichroism Data at a Concentration $1 \mathrm{mg} / \mathrm{mL}$ (Figure 8)}
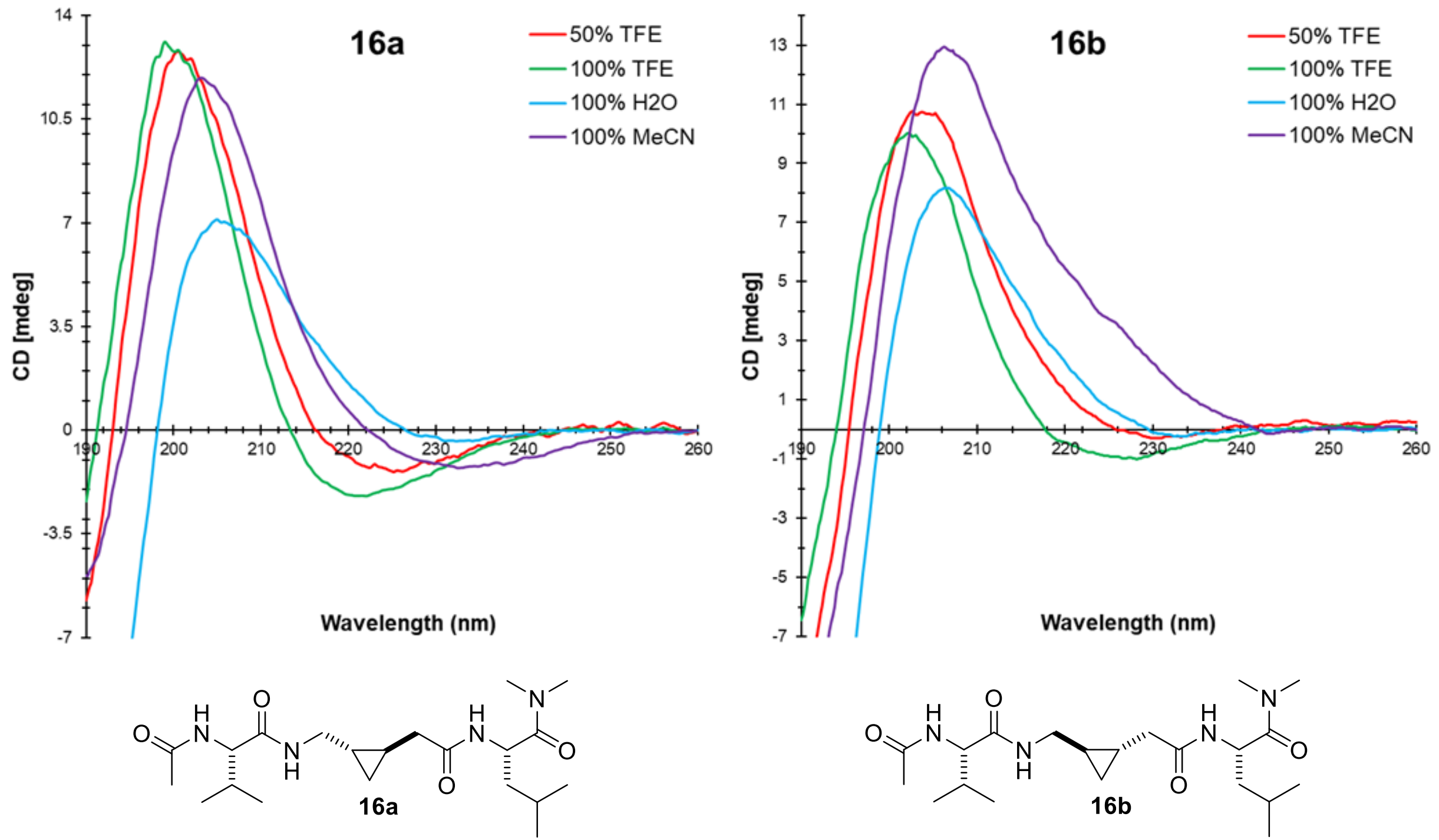